\title{
WestVirginiaUniversity
}

THE RESEARCH REPOSITORY @ WVU

Graduate Theses, Dissertations, and Problem Reports

2007

\section{De novo asymmetric synthesis of digitoxin based carbohydrate libraries}

Wenjun Xin

West Virginia University

Follow this and additional works at: https://researchrepository.wvu.edu/etd

\section{Recommended Citation}

Xin, Wenjun, "De novo asymmetric synthesis of digitoxin based carbohydrate libraries" (2007). Graduate Theses, Dissertations, and Problem Reports. 4351.

https://researchrepository.wvu.edu/etd/4351

This Thesis is protected by copyright and/or related rights. It has been brought to you by the The Research Repository @ WVU with permission from the rights-holder(s). You are free to use this Thesis in any way that is permitted by the copyright and related rights legislation that applies to your use. For other uses you must obtain permission from the rights-holder(s) directly, unless additional rights are indicated by a Creative Commons license in the record and/ or on the work itself. This Thesis has been accepted for inclusion in WVU Graduate Theses, Dissertations, and Problem Reports collection by an authorized administrator of The Research Repository @ WVU. For more information, please contact researchrepository@mail.wvu.edu. 
De Novo Asymmetric Synthesis of Digitoxin Based

\title{
Carbohydrate Libraries
}

\author{
Wenjun Xin \\ Thesis submitted to the \\ Eberly College of Arts and Sciences \\ at West Virginia University \\ in partial fulfillment of the requirements \\ for the degree of \\ Master of Science \\ In \\ Organic Chemistry
}

George A. O’Doherty, Ph.D., Chair

Björn C. Söderberg, Ph.D.

Kung K. Wang, Ph.D.

C. Eugene Bennett Department of Chemistry

Morgantown, West Virginia

2007

Keywords: Digitoxin

Copyright 2007 Wenjun Xin 


\section{ABSTRACT \\ De Novo Asymmetric Synthesis of Digitoxin Based Carbohydrate Libraries Wenjun Xin}

The enantioselective syntheses of digitoxigen 2,6-dideoxy- $\beta$-L-ribo-hexopyranoside, its analogues and digitoxin monosaccharide analogues have been achieved. Key to this approach is the iterative application of the palladium-catalyzed glycosylation reaction, reductive 1,3-transposition and diastereoselective dihydroxylation. Anticancer tests showed that these analogues have even better anticancer activities. 


\section{DEDICATED TO}

My mother Suzhen Ye and my father Dingyun Xin 


\section{ACKNOWLEDGEMENT}

First of all, I would like to give my sincere gratitude to my advisor, George A. O'Doherty, for his great support, patience, and encouragement throughout my graduate studies. His mentorship was essential to the completion of this thesis.

My thanks also go to Dr. Kung Wang and Dr. Björn C. Söderberg for their valuable comments that improved the contents of this thesis.

I would also like to thank the former and present group members, Dr. Md. M. Ahmed, Dr. Joseph Dougherty, Dong Gao, Miaosheng Li, Maoquan Zhou, Sannjev Guppi, Matt Mortensen, Haibing Guo, Hu Cui, Mingde Shan, Philip Harsh, Xiaomei Yu for their help and company and the entire faculty, staff, and graduate students for their friendship and help in a variety of ways.

Financial support from the National Institute of Health, C. Eugene Bennett Department of Chemistry at West Virginia University, the National Science Foundation, NSF-EPSCoR (0314742) for a $600 \mathrm{MHz}$ NMR at WVU are gratefully acknowledged.

Finally, and most importantly, I would like to thank my parents for their love and support for my education through the past many years. 


\section{TABLE OF CONTENTS}

Title Page

Abstract

$\begin{array}{ll}\text { Dedications } & \text { iii }\end{array}$

Acknowledgement iv

Table of Contents $\quad \mathrm{V}$

List of Figures $\quad$ vi

List of Schemes $\quad$ vii

List of Abbreviations $\quad$ viii

\section{CHAPTER I}

Asymmetric Synthesis of Digitoxin Sugar Analogues

1.1. Introduction 1

1.2. Previous approaches to digitoxin 2

1.3. O'Doherty's synthesis of digitioxin 3

1.4. Total synthesis of digitoxigen 2,6- $\beta$-L-ribo-hexopyranoside and its $\begin{array}{ll}\text { analogues } & 4\end{array}$

1.4.1. Background 4 
1.4.2. Retrosynthetic analysis

1.4.3. Synthesis of Boc-pyranones $\quad 7$

1.4.4. Synthesis of digitoxigen 2,6-dideoxy- $\beta$-L-ribo-hexopyranoside 8

1.4.5. Synthesis of digitoxigen 2,6-dideoxy- $\beta$-L-ribo-hexopyranoside analogues

1.4.6. Anticancer tests results of digitoxin monosaccharide sugar analogues

1.4.7. Synthesis of digitoxigen 2,6-dideoxy- $\alpha$-D-ribo-hexopyranoside analogues

1.4.8. Synthetic effort towards the L- $\beta$-glycoside of desacetoxyoleandrin

1.4.9. Conclusion

\section{CHAPTER II}

\section{Experimental Section}

Instrumentation, Materials and Manipulation

References

Appendix

\section{List of Figures}

Figure 1. Digitoxin, digitoxingenin and digoxose. 
Figure 2. Anticancer tests results of digitoxin mono-, di- and tri-saccharide.

Figure 3. The L-sugar digitoxin mono-, di- and tri-saccharide.

Figure 4. Digitoxin monosaccharide sugar analogues.

Figure 5. Anticancer tests results of digitoxin monosaccharide sugar analogues. 13

Figure 6. Oleandrin, the L- $\beta$-glycoside of desacetoxy-oleandrin and digitoxigen 2,6-dideoxy- $\beta$-L-ribo-hexopyranoside.

\section{List of Schemes}

Scheme 1. Retrosynthetic analysis of Wiesner's synthesis.

Scheme 2. Retrosynthetic analysis of McDonald's synthesis.

Scheme 3. Retrosynthetic analysis of O'Doherty's synthesis.

Scheme 4. Retrosynthetic analysis of L-sugar digitoxin.

Scheme 5. Enantio- and diastereoselective pyranone synthesis.

Scheme 6. Synthesis of digitoxigen 2,6-dideoxy- $\beta$-L-ribo-hexopyranoside.

Scheme 7. Synthesis of digitoxigen 2,6-dideoxy- $\beta$-L-gluco-hexopyranoside.

Scheme 8. Synthesis of digitoxigen rhamno analogues.

Scheme 9. Synthesis of digitoxigen 2,6-dideoxy- $\alpha$-D-ribo-hexopyranoside analogues.

Scheme 10. Synthetic effort towards the L- $\beta$ glycoside of desacetoxyoleandrin. 


\section{List of Abbreviations}

Ac

Boc

DBA

DEAD

DMAP

DMF

Et

EtOAc

IR

$\mathrm{Me}$

$\mathrm{MHz}$

mmol

MS

NBS

NBSH

NMR

NMO

$\mathrm{Ph}$

$\mathrm{PMBz}$

$\mathrm{PNBz}$
Acetyl

$N$-tert-butoxycarbonyl

trans, trans-dibenzylideneacetone

Diethyl azodicarboxylate

4-Dimethylaminopyridine

$N, N$-Dimethylformamide

Ethyl

Ethyl acetate

Infrared

Methyl

megahertz

millimole(s)

Mass spectrum

$N$-Bromosuccinimide

$o$-Nitrobenzenesulfonylhydrazide

Nuclear magnetic resonance

$N$-methylmorpholine

Phenyl

para-Methoxybenzyl

p-nitrobenzene 
tetrahydrofuran

TLC

Thin layer chromatography 


\section{Appendix}

\section{List of ${ }^{1} \mathrm{H}$ and ${ }^{13} \mathrm{C}$ NMR Spectra}

${ }^{1} \mathrm{H}$ and ${ }^{13} \mathrm{C}$ NMR Spectra of Compound 23

$40-41$

${ }^{1} \mathrm{H}$ and ${ }^{13} \mathrm{C}$ NMR Spectra of Compound 24

$42-43$

${ }^{1} \mathrm{H}$ and ${ }^{13} \mathrm{C}$ NMR Spectra of Compound 25

$44-45$

${ }^{1} \mathrm{H}$ and ${ }^{13} \mathrm{C}$ NMR Spectra of Compound 15

$46-47$

${ }^{1} \mathrm{H}$ and ${ }^{13} \mathrm{C}$ NMR Spectra of Compound 30

$48-49$

${ }^{1} \mathrm{H}$ and ${ }^{13} \mathrm{C}$ NMR Spectra of Compound 26

$50-51$

${ }^{1} \mathrm{H}$ and ${ }^{13} \mathrm{C}$ NMR Spectra of Compound 31

$52-53$

${ }^{1} \mathrm{H}$ and ${ }^{13} \mathrm{C}$ NMR Spectra of Compound 27

$54-55$

${ }^{1} \mathrm{H}$ and ${ }^{13} \mathrm{C}$ NMR Spectra of Compound 28

$56-57$

${ }^{1} \mathrm{H}$ and ${ }^{13} \mathrm{C}$ NMR Spectra of Compound 29

58-59

${ }^{1} \mathrm{H}$ and ${ }^{13} \mathrm{C}$ NMR Spectra of Compound 32

60-61

${ }^{1} \mathrm{H}$ and ${ }^{13} \mathrm{C}$ NMR Spectra of Compound 33

$62-63$

${ }^{1} \mathrm{H}$ and ${ }^{13} \mathrm{C}$ NMR Spectra of Compound 34

64-65

${ }^{1} \mathrm{H}$ and ${ }^{13} \mathrm{C}$ NMR Spectra of Compound 36

66-67 


\section{CHAPTER I}

\section{Asymmetric Synthesis of Digitoxin Sugar Analogues}

\subsection{Introduction}

Digitoxin (1) (Figure 1) is a cardiac glycoside which can be extracted from the leaves of Digitalis purpurea (purple foxglove). It has long been used to slow the heart rate while increasing the contractility of the heart muscle (inotropic activity). It has been widely prescribed for congestive heart failure and cardiac arrhythmia for over 200 years. Oligosaccharides bearing deoxysugars have played a pivotal role in many pharmacologically important antibiotics, vaccines and antitumor agents. ${ }^{1}$ Digitoxin has also been shown to possess potential anticancer activities. ${ }^{2}$ The natural product digitoxin contains three D digitose sugars. Structurally, digitoxin is the combination of two natural products, the aglycon digitoxigenin $(2)^{3}$ and the trisaccharide digoxose (3). ${ }^{4}$

Figure 1. Digitoxin, digitoxigenin and digoxose.

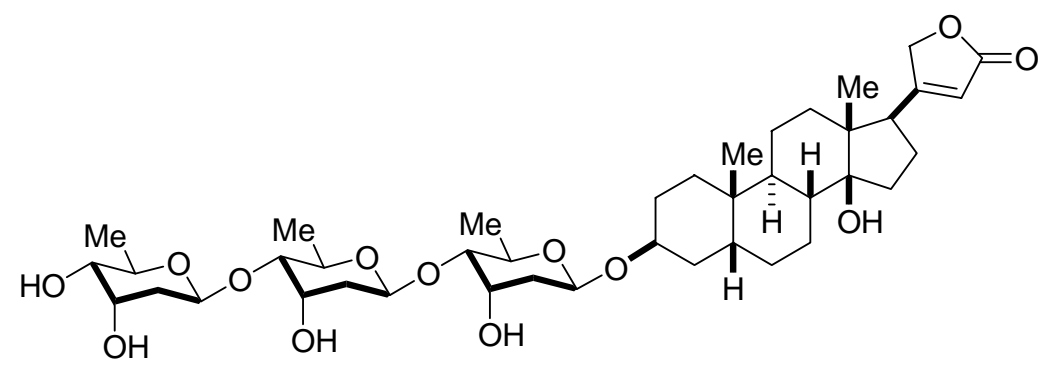

digitoxin (1)

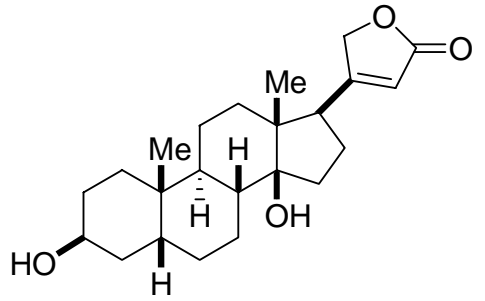

digitoxigenin (2)

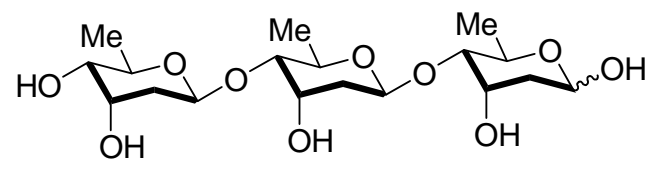

digoxose (3) 


\subsection{Previous approaches to digitoxin}

There have been two syntheses of digitoxin from the aglycone, a carbohydrate approach by Wiesner ( 20 steps from a protected 2-deoxy sugar) and a de novo approach by McDonald (20 steps from TMS-acetylene). ${ }^{5}$ There have been 8 syntheses of digitoxigenin. $^{6}$

The first synthesis of digitoxin from digitoxigenin was accomplished by Wiesner's group (Scheme 1). The stereochemistry of the digitoxin glycoside bond was controlled by a 1,3-participation of PMBz ester (4). The PMBz ester (4) was derived from a 1,3-participation of $N$-methylurethane (5) which was derived from bromide (6) in 7 steps. In turn, bromide (6) was prepared from methyl $\alpha$-D-glucoside.

Scheme 1. Retrosynthetic analysis of Wiesner's synthesis.

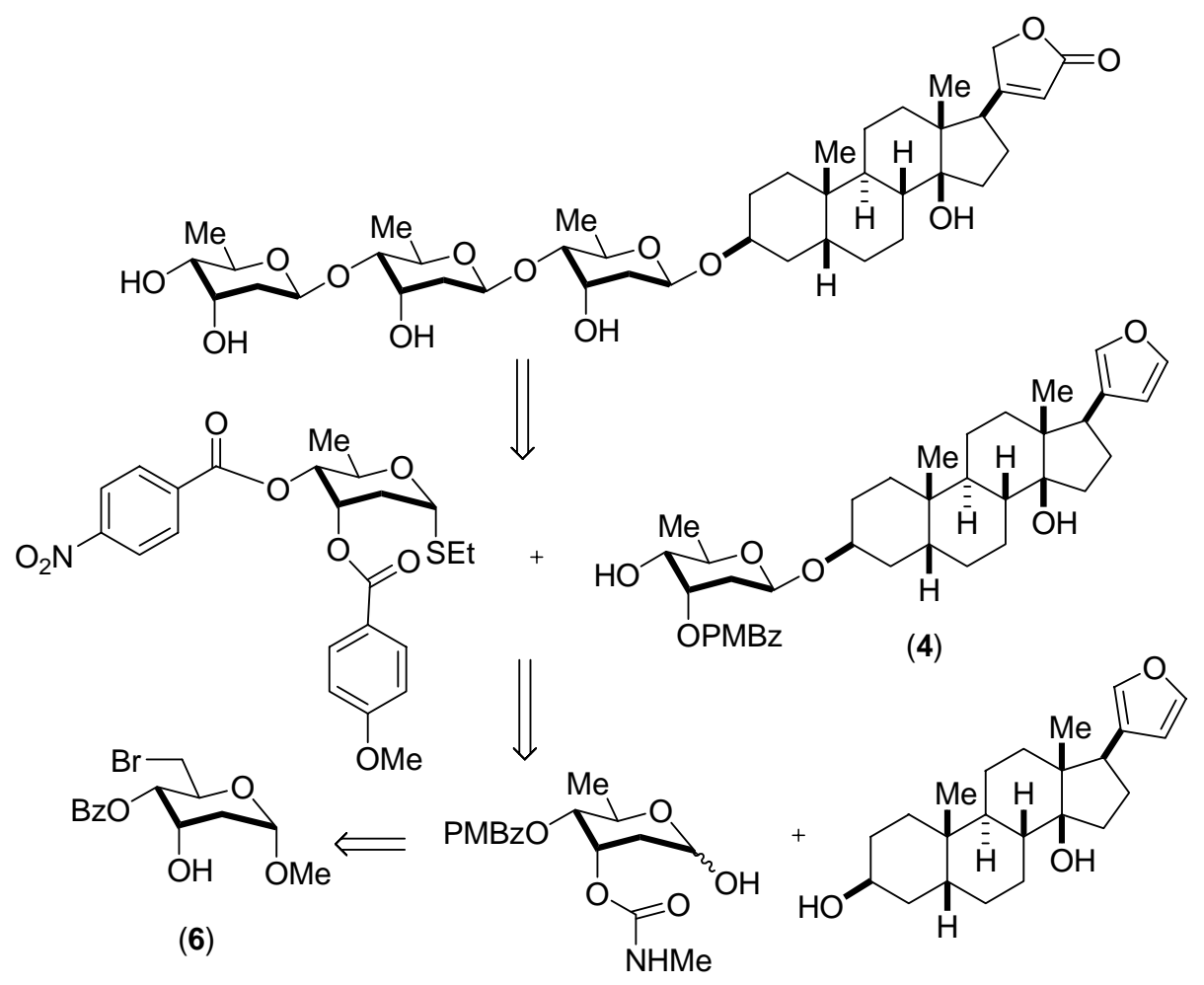

(5) 
McDonald's synthesis (Scheme 2) achieved the product digitoxin by the acid catalyzed glycosylation of digitoxigenin and trisaccharide glycal (7). The trisaccharide glycal (7) was derived from 6-deoxy-D-riboglycal (8) and alkynyl alcohol (9) through an acid catalyzed glycosylation. Furthermore, these two compounds were derived from alkynyl enone (10) in seven and five steps, respectively.

Scheme 2. Retrosynthetic analysis of McDonald's synthesis.

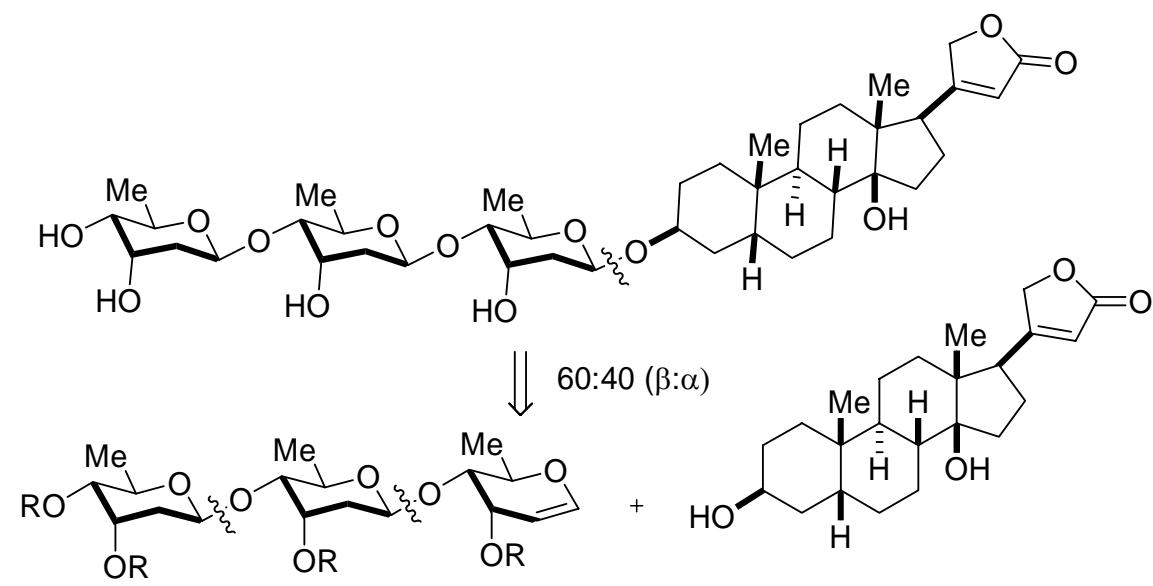

(7)

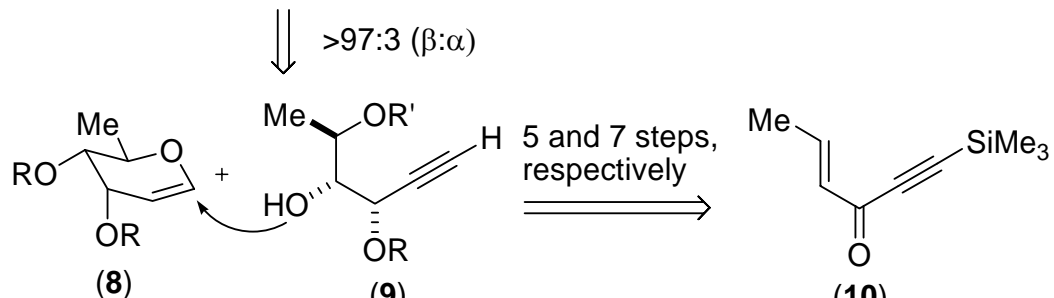

(8)

(9)

(10)

tungsten carbonyl catalyzed cycloisomerization

\subsection{O'Doherty's synthesis of digitoxin}

O’Doherty's group also prepared digitoxin via a de novo strategy, which could also be used to prepare various digitoxin analogues (Scheme 3). ${ }^{7}$ Due to the missing stereo-control element at the 2-position, it is particularly difficult to synthesize $\beta$-2deoxy-glycosides. They successfully addressed the 2-deoxy- $\beta$-glycosides using a diastereoselective palladium-catalyzed glycosylation reaction. Digitoxin was derived 
from digitoxin disaccharide (11), which was produced from a corresponding digitoxin monosaccharide. This digitoxin monosaccharide was prepared from digitoxigenin and Boc-pyranone (13ß) via a palladium-catalyzed glycosylation reaction. Boc-pyranone (13ß) was derived from the commercially available compound acylfuran (14). Comparing with the previous approaches, the O'Doherty's approach is a more efficient route in terms of number of steps and stereocontrol. In addition, this route is more amenable.

Scheme 3. Retrosynthetic analysis of O’Doherty's synthesis.
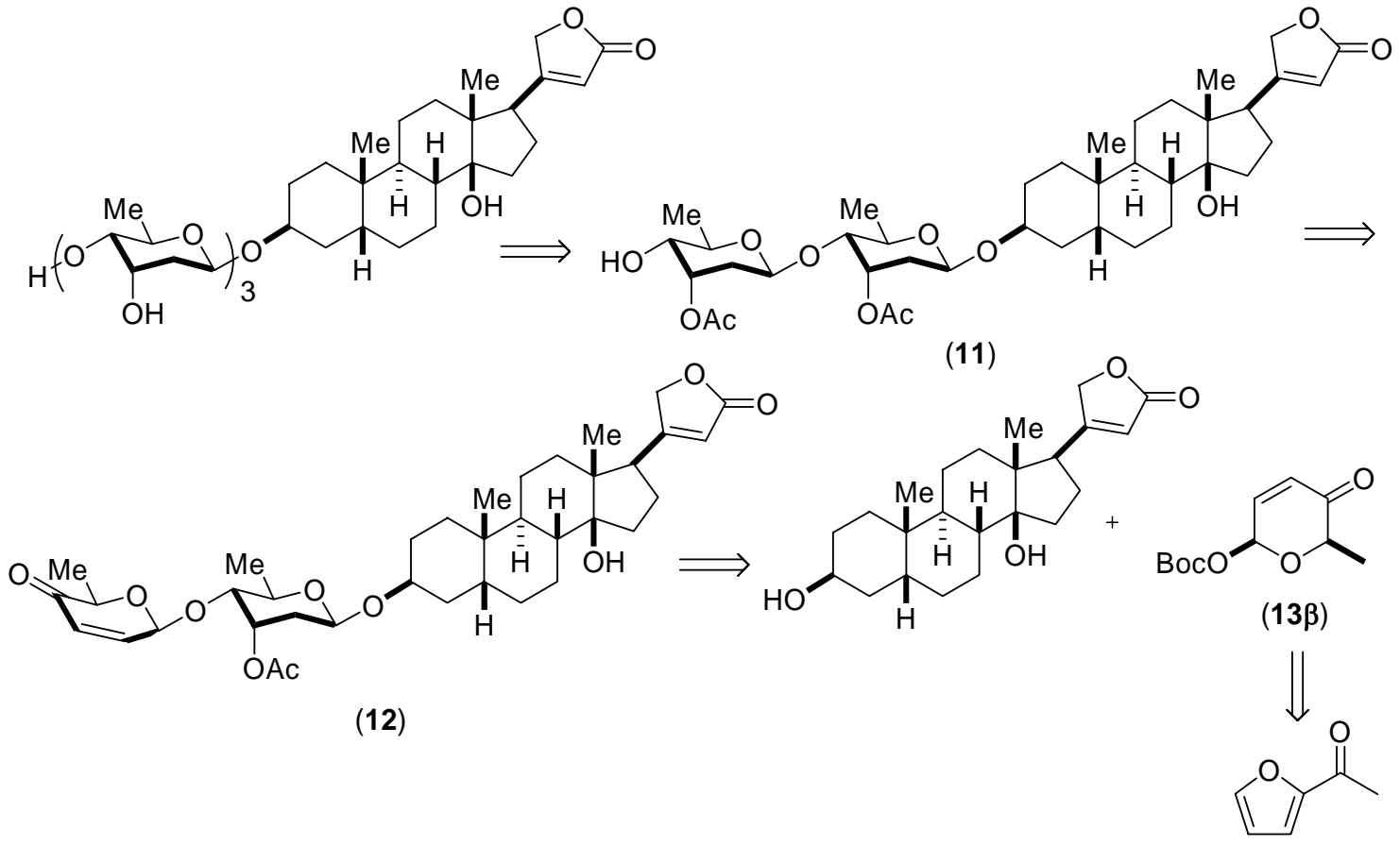

(14)

\subsection{Total synthesis of digitoxigen 2,6- $\beta$-L-ribo-hexopyranoside and its analogues}

\subsubsection{Background}

Previously, O'Doherty's group successfully synthesized and tested the natural compound digitoxin and its mono- and di-saccharide analogues as antitumor agents. The biological tests of these compounds from NCI (National Cancer Institute) showed that in 
general the digitoxin monosaccharide is more active than digitoxin or the digitoxin disaccharide in cancer cell cytoxicity (Figure 2) ${ }^{8}$ To further understand this phenomenon, we were interested in synthesizing several unnatural L-sugar digitoxin analogues (figure 3) with the particular goal of finding the most active anticancer agent.

Figure 2. Anticancer tests results of digitoxin mono-, di- and tri-saccharide.

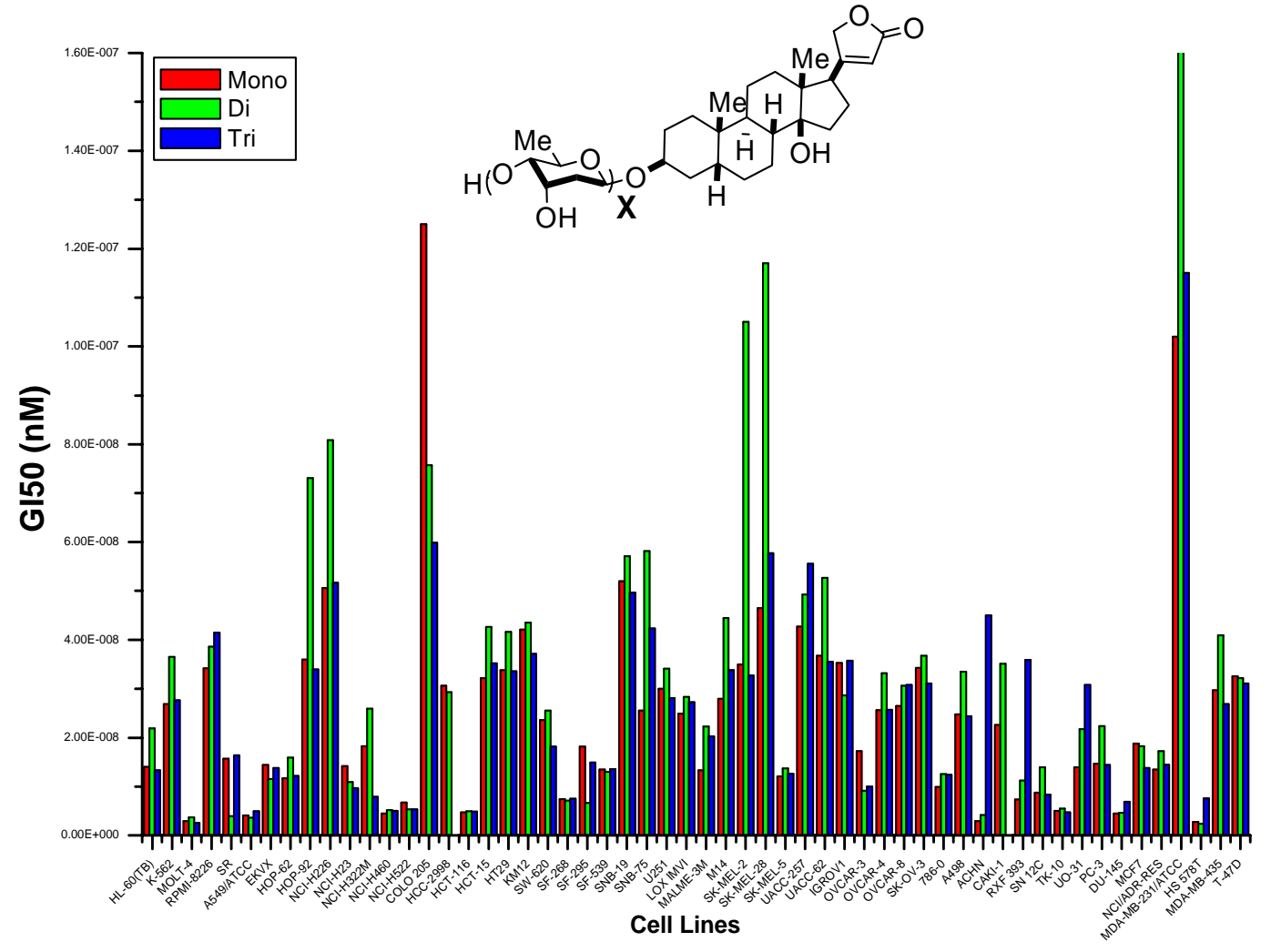


Figure 3. The L-sugar digitoxin mono-, di- and tri-saccharide.

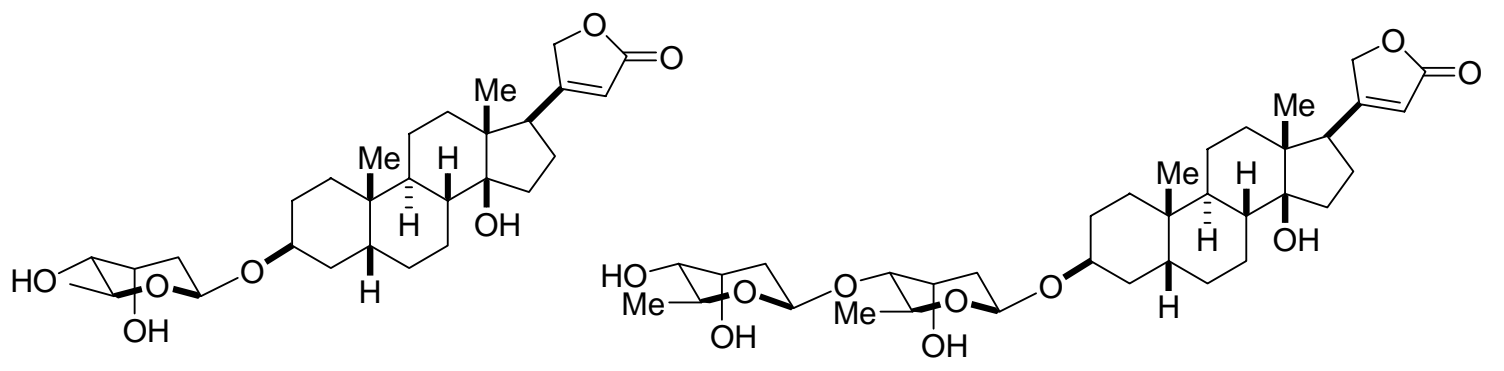

L-sugar digitoxin monosaccharide (15)

L-sugar digitoxin disaccharide (16)

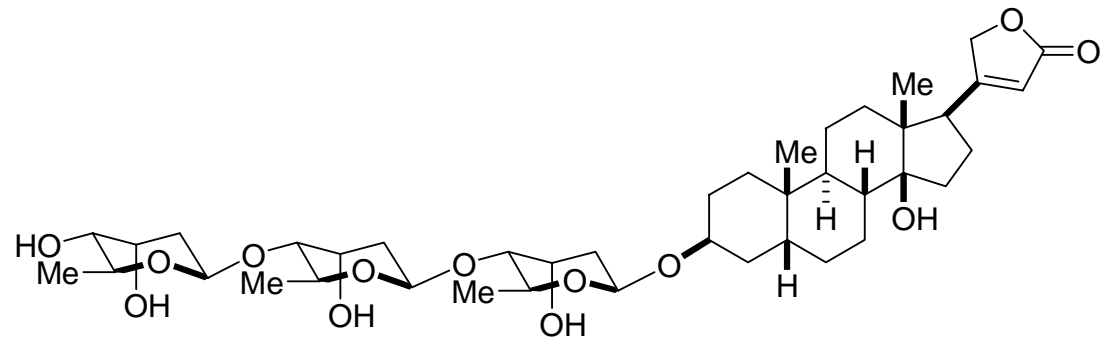

L-sugar digitoxin (17)

\subsubsection{Retrosynthetic analysis}

A similar approach was planed to synthesize these L-sugar digitoxin diastereomeric analogues (Scheme 4). The final product, L-sugar digitoxin trisaccharide could be derived from L-sugar digitoxin disaccharide through a palladium-catalyzed glycosylation reaction. The disaccharide could also be prepared from the L-sugar digitoxin monosaccharide via a palladium-catalyzed glycosylation reaction. The monosaccharide could be produced from the palladium-catalyzed glycosylation of digitoxigenin and L-Boc-pyranone (18ß). From previous work, it was known that the Boc-pyranone (18ß) could be derived from acylfuran. 
Scheme 4. Retrosynthetic analysis of L-sugar digitoxin.

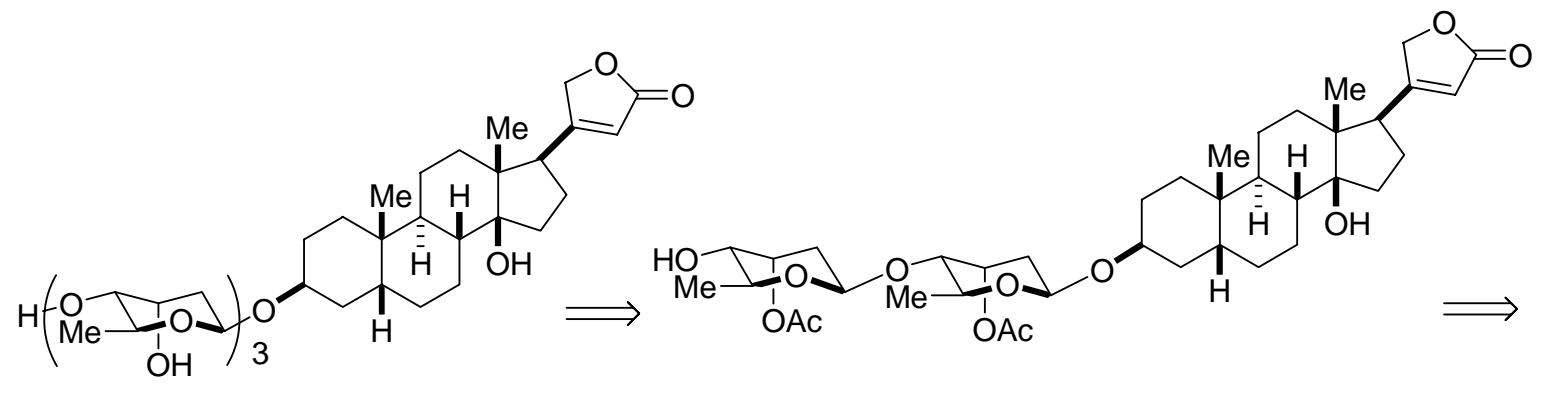

L-sugar digitoxin (17)
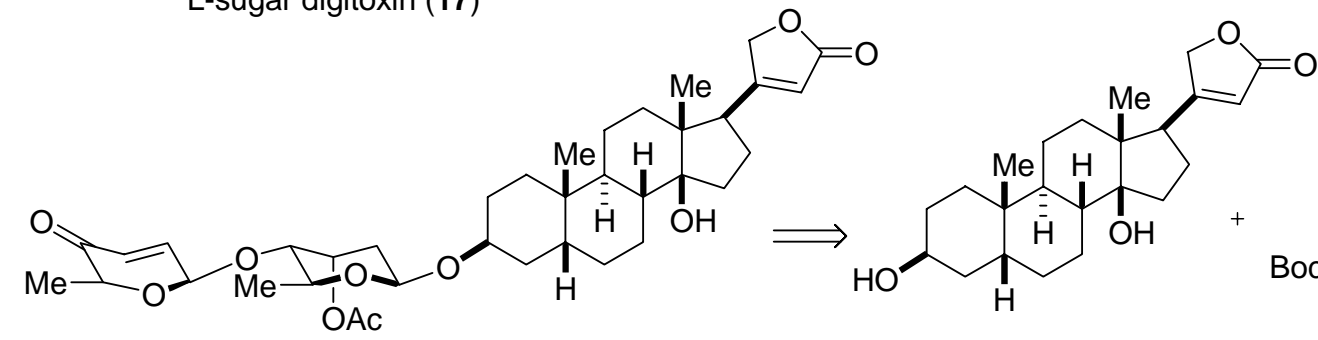<smiles>C[C@@H]1O[C@@H](OC(C)(C)C)C=CC1=O</smiles>

digitoxigenin

$(\mathbf{1 8 \beta )}$

(2)

\subsubsection{Synthesis of Boc-pyranones}

According to the O’Doherty group's previous approach, acylfuran (14) could be enantioselectively reduced (Noyori $(\mathrm{S}, \mathrm{S}),>95 \%$ ee) $)^{9}$ to furan alcohol (19) and converted into the L-sugar pyranone (20) and then diastereoselectively acylated into the $\alpha$-Boc-

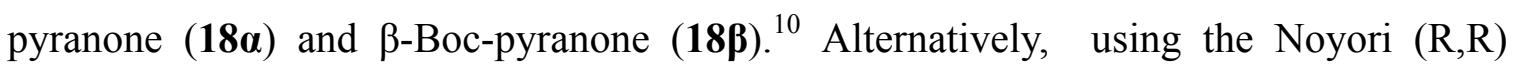
catalyst would provide the enantiomeric furan alcohol (21) and similarly lead to the two D-sugar diastereometers $(\mathbf{1 3 \alpha})$ and $(\mathbf{1 3 \beta})$. When the Boc-protection was performed at -78 ${ }^{\circ} \mathrm{C}$, the $\alpha: \beta$ ratio is $3: 1$. When the Boc-protection was performed at elevated temperature $\left((\mathrm{Boc})_{2} \mathrm{O} / \mathrm{NaOAc}\right.$ in benzene at $\left.80{ }^{\circ} \mathrm{C}\right)$, a switch in the diastereoselectivity occurred (Scheme 5). The ratio of $\beta$-pyranone to $\alpha$-pyranone at these higher temperatures could be as high as 1.3:1. 
Scheme 5. Enantio-and diastereoselective pyranone synthesis.

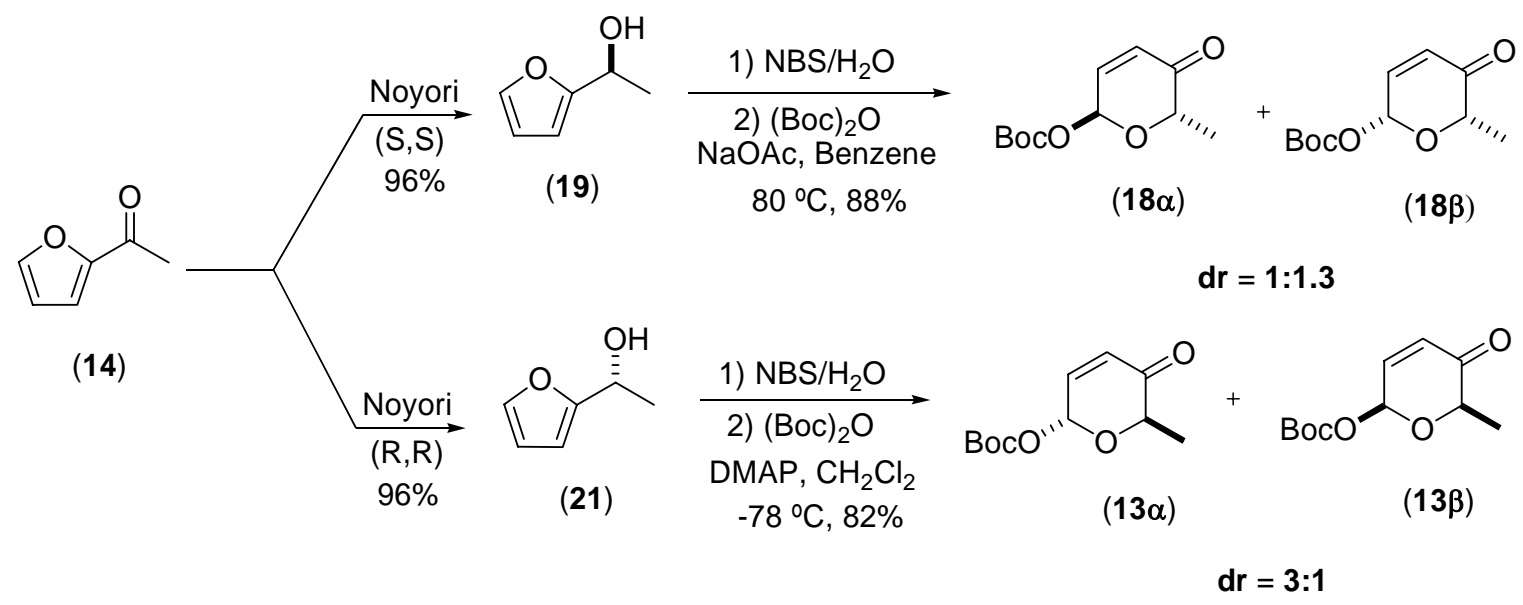

\subsubsection{Synthesis of digitoxigen 2,6-dideoxy- $\beta$-L-ribo-hexopyranoside}

Starting from $\beta$-Boc-pyranone (18ß), a palladium-catalyzed glycosylation reaction was performed between digitoxigenin (2) and Boc-pyranone (18ß) which gave pyranone (23) in $85 \%$ yield. Pyranone (23) was then reduced with $\mathrm{NaBH}_{4}$ to obtain allylic alcohol (24) in $81 \%$ yield. The reductive rearrangement gave olefin (25) in $98 \%$ yield. The dihydroxylation of olefin (25) achieved the L-sugar digitoxin monosaccharide (15) in 93\% yield (Scheme 6). 
Scheme 6. Synthesis of digitoxigen 2,6-dideoxy- $\beta$-L-ribo-hexopyranoside.

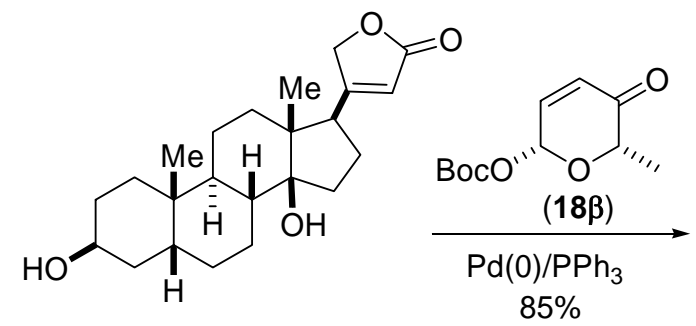

digitoxigenin

(2)

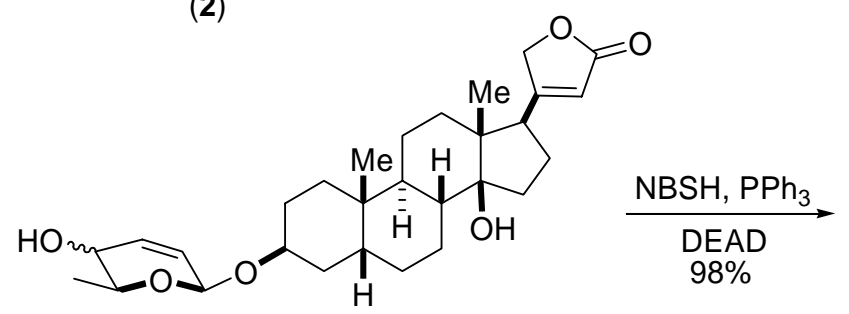

(24)

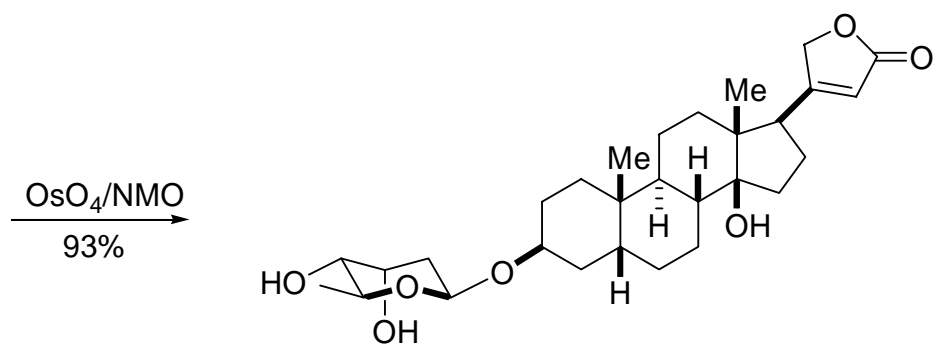

digitoxigen 2,6-dideoxy- $\beta$-L-ribo-hexopyranoside

(15)

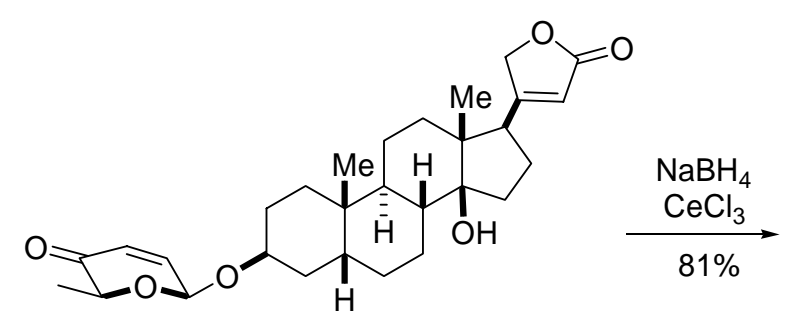

(23)

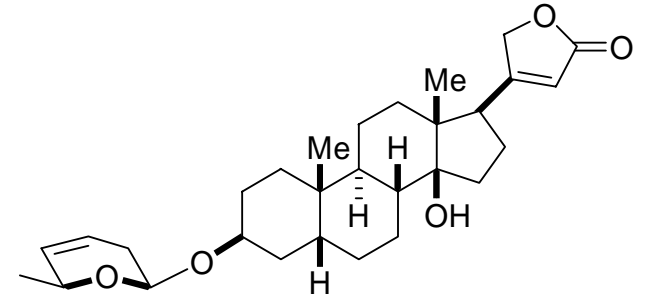

(25)

\subsubsection{Synthesis of digitoxigen 2,6-dideoxy- $\beta$-L-ribo-hexopyranoside analogues}

From the L-sugar digitoxin monosaccharide (15), digitoxigen 2,6-dideoxy- $\beta$-Lgluco-hexopyranoside (26) was synthesized (Figure 4). 
Figure 4. Digitoxin monosaccharide sugar analogues.

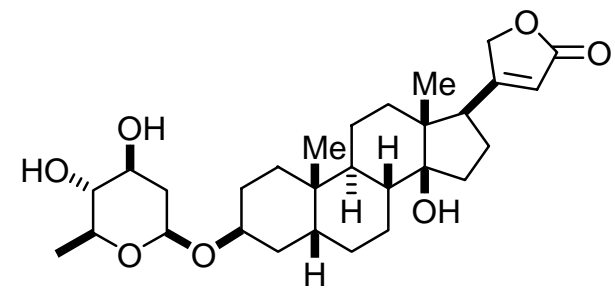

digitoxigen 2,6-dideoxy- $\beta$-L-gluco-hexopyranoside

(26)

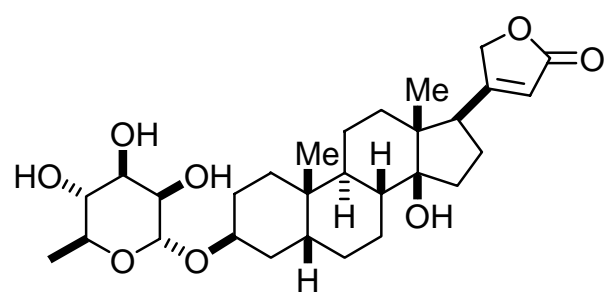

digitoxingen $\alpha$-L-rhamno-hexopyranoside

(28)

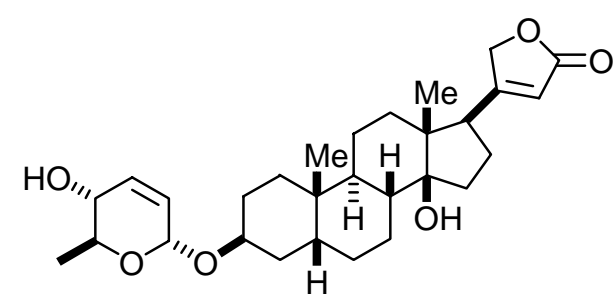

digitoxigen 2,3-dideoxy- $\alpha$-L-rhamno-hexopyranoside

(27)

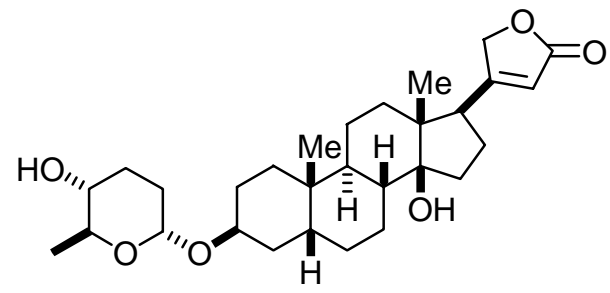

digitoxigen 2,3-dideoxy-2,3-dihydro$\alpha$-L-rhamno-hexopyranoside

(29)

Mitsunobu reaction ${ }^{11}$ of the L-sugar digitoxin monosaccharide (15) gave the C-3 inverted ester (30) in $85 \%$ yield. Hydrolysis of (30) with $\mathrm{MeOH} / \mathrm{Et}_{3} \mathrm{~N}$ provided the diastereomeric monosaccharide digitoxigen 2,6-dideoxy- $\beta$-L-gluco-hexopyranoside (26) in good yield (Scheme 7). 
Scheme 7. Synthesis of digitoxigen 2,6-dideoxy- $\beta$-L-gluco-hexopyranoside.

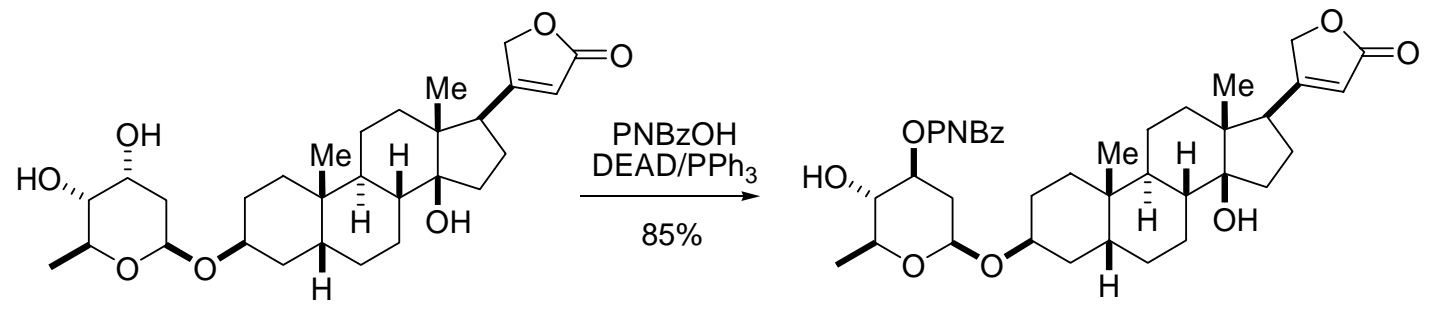

digitoxigen 2,6-dideoxy- $\beta$-L-ribo-hexopyranoside

(30)

(15)

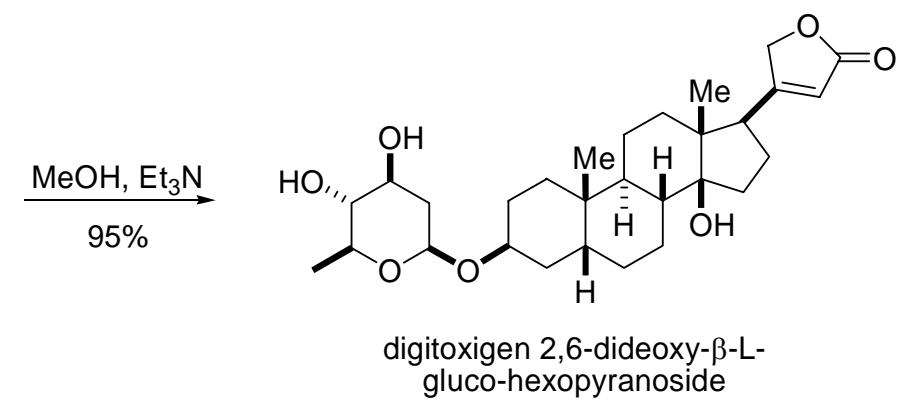

(26)

The palladium-catalyzed glycosylation reaction of digitoxigenin (2) and $\alpha$-Boc-

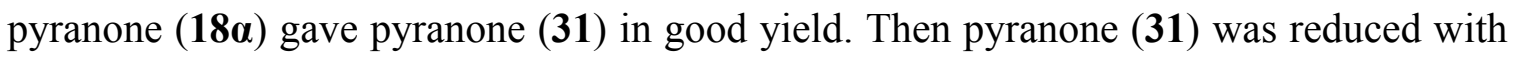
$\mathrm{NaBH}_{4}$ to obtain analogue digitoxigen 2,3-dideoxy- $\alpha$-L-rhamno-hexopyranoside (27) in 83\% yield. Dihydroxylation of (27) gave the rhamno analogue (28). Reductive rearrangement of (27) gave C-2/C-3 dideoxy analogue (29) in 96\% yield (Scheme 8). 
Scheme 8. Synthesis of digitoxigen rhamno analogues.

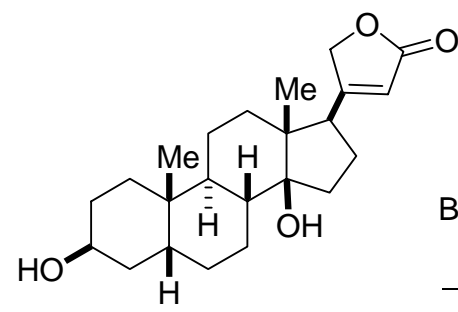

digitoxigenin

(2)
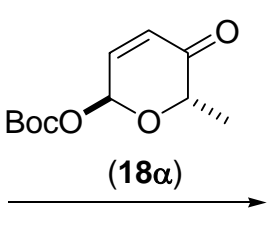

$\mathrm{Pd}(0) / \mathrm{PPh}_{3}$

$86 \%$

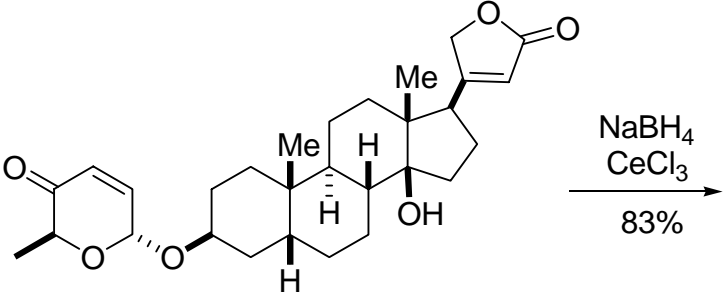

(31)

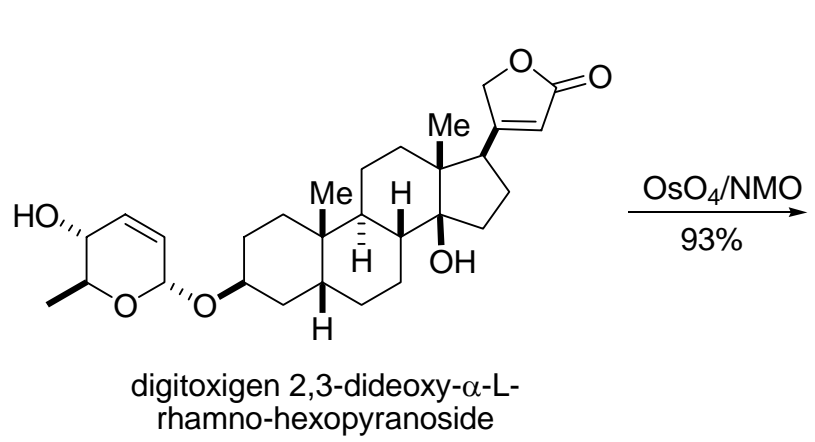

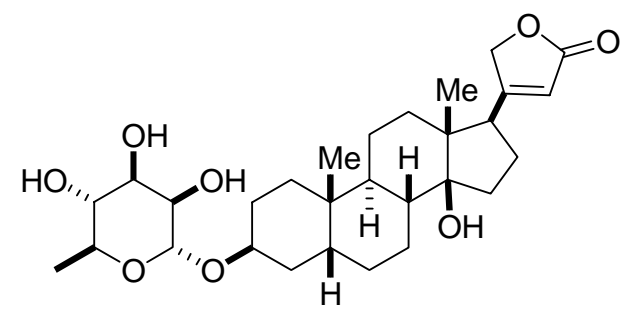

digitoxingen $\alpha$-L-rhamno-hexopyranoside

(27)
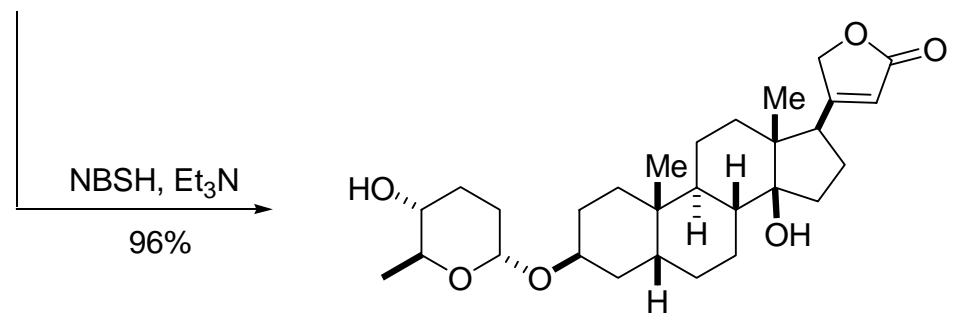

digitoxigen 2,3-dideoxy-2,3-dihydro$\alpha$-L-rhamno-hexopyranoside

(29)

\subsubsection{Anticancer tests results of digitoxin monosaccharide sugar analogues}

After having successfully synthesized several L-sugar digitoxin monosaccharide analogues, these compounds were sent for anticancer testing. ${ }^{12}$ Tests results showed that these analogues have excellent anticancer activities. In fact, analogue (28) shows the best anticancer activity comparing with other analogues. For instance, (28) is 13.5 times more active than digitoxin in killing HT-29 cancer cell lines. Analogues (15), (26), (29) also 
show much better activities comparing with digitoxin although the difference isn't as great (Figure 5).

Figure 5. Anticancer tests results of digitoxin monosaccharide sugar analogues.

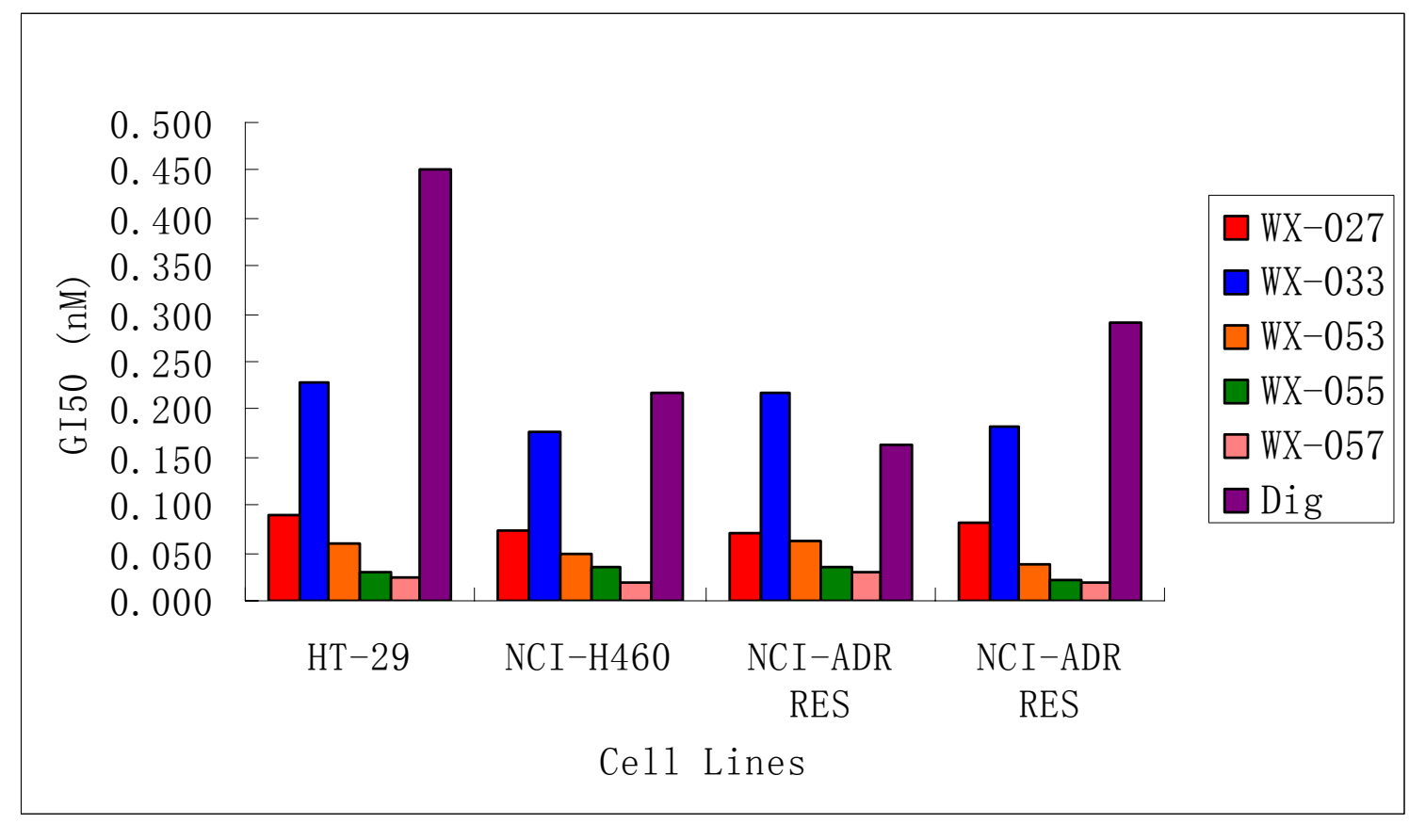

WX-027: (15) WX-033: (26) WX-053: (27) WX-055: (29) WX-057: (28)

Dig: digitoxin (1)

\subsubsection{Synthesis of digitoxigen 2,6-dideoxy-a-D-ribo-hexopyranoside analogues}

Starting with Boc-pyraone (13a), a number of D-sugar digitoxin monosaccharide analogues have been achieved (Scheme 9). The palladium-catalyzed glycosylation reaction of digitoxigenin (2) and $\alpha$-Boc-pyranone (13a) gave pyranone (32) in good yield. Then pyranone (32) was reduced with $\mathrm{NaBH}_{4}$ to obtain the digitoxigen 2,3dideoxy- $\alpha$-D-rhamno-hexopyranoside (33) in $90 \%$ yield. Reductive rearrangement of (33) gave the dideoxy analogue (34) in 95\% yield. 
Scheme 9. Synthesis of digitoxigen 2,6-dideoxy- $\alpha$-D-ribo-hexopyranoside analogues.

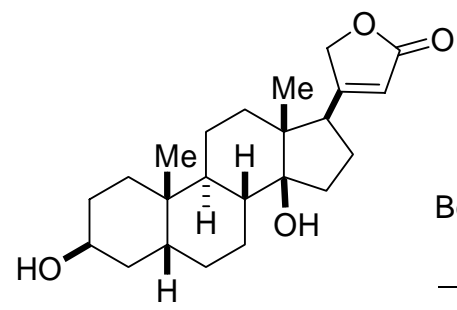

digitoxigenin

(2)

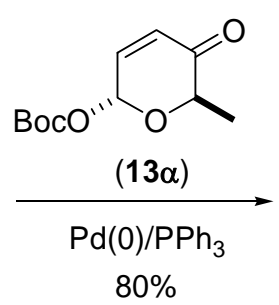

$80 \%$

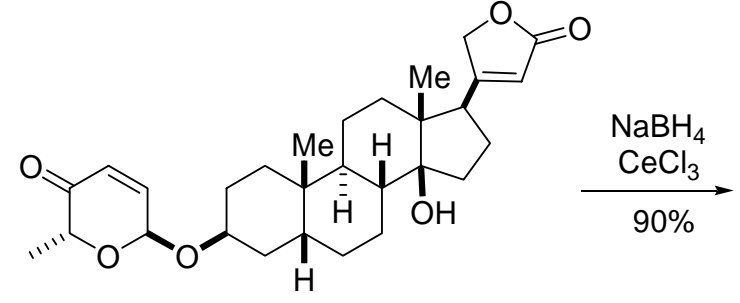

(32)

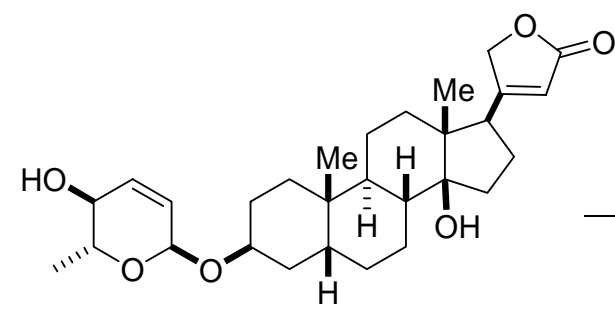

(33)

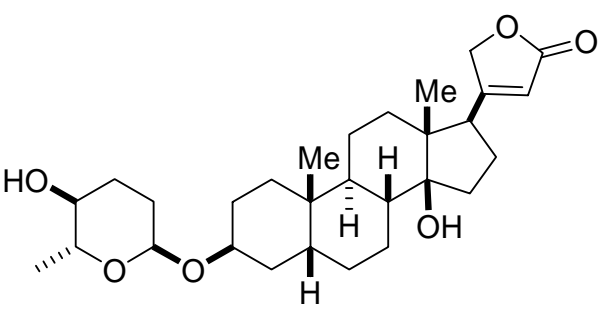

(34)

\subsubsection{Synthetic effort towards the L- $\beta$-glycoside of desacetoxy-oleandrin}

Oleandrin (35) (Figure 6) is another cardiac glycoside which can be extracted from the leaves of one of the very poisonous plants oleander. The structures of digitoxin monosaccharide and $\beta$-oleandrin are very similar, with the addition of a C-16 acetoxy group and a C-3 methylated D-2-deoxy glucose sugar. We are interested in synthesizing an L-sugar $\beta$-desacetoxy-oleandrin from digitoxin monosaccharide and comparing their anticancer activities. 
Figure 6. Oleandrin, the L- $\beta$-glycoside of desacetoxy-oleandrin and digitoxigen 2,6dideoxy- $\beta$-L-ribo-hexopyranoside.

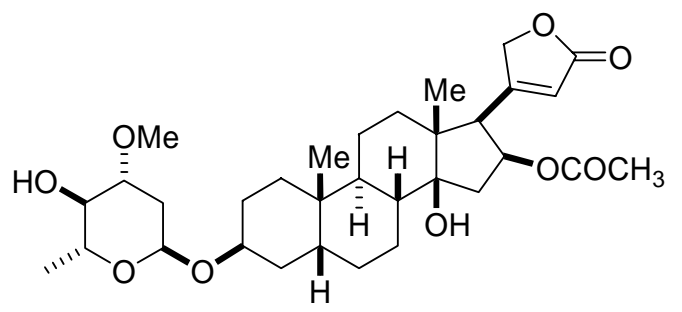

oleandrin (35)

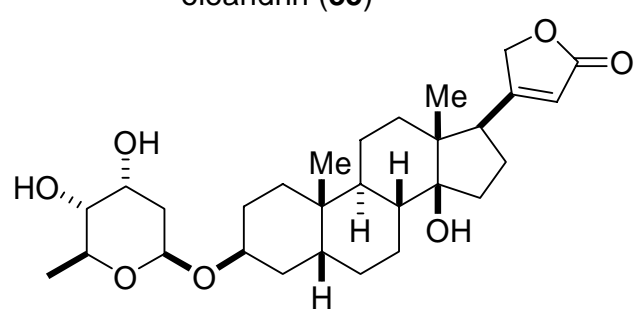

L-sugar digitoxin monosaccharide (15)

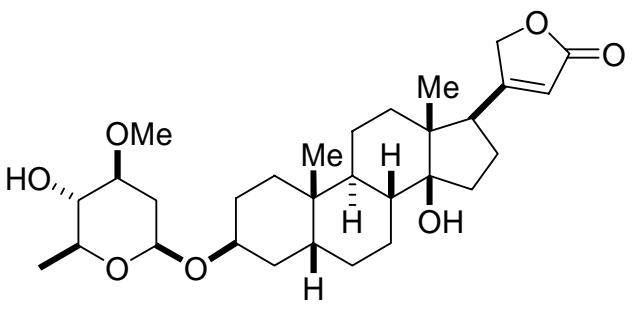

L- $\beta$-gycoside desacetoxy-oleandrin (36)

The synthesis of digitoxigen 2,6-dideoxy- $\beta$-L-gluco-hexopyranoside (26) has already been achieved in good yield. Thus the synthesis of analogue (36) began with the direct methylation of $(\mathbf{2 6})^{13}$ to selectively prepare the L- $\beta$-glycoside of desacetoxyoleandrin (36) (Scheme 10).

Scheme 10. Synthetic effort towards the L- $\beta$-glycoside of desacetoxy-oleandrin.

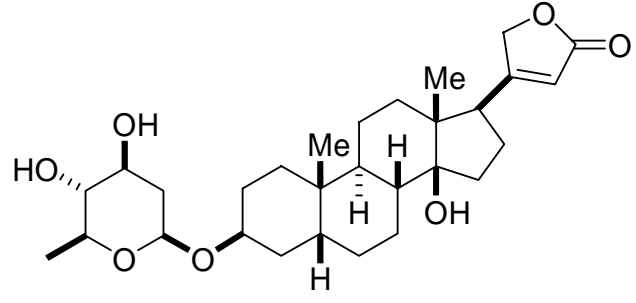

digitoxigen 2,6-dideoxy- $\beta$-L-gluco-hexopyranoside (26)

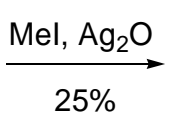

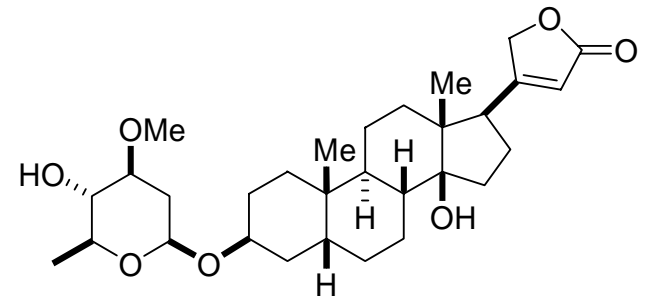

L- $\beta$-glycoside desacetoxy-oleandrin (36)

However, this methylation reaction was not very selective. The highest yield was $25 \%$ by treating $\beta$-2,6-dideoxy-L-gluco-digitoxin-monosaccharide (26) with $\mathrm{MeI}$ and $\mathrm{Ag}_{2} \mathrm{O}$, which yielded a mixture of regioisomers. 


\subsubsection{Conclusion}

A highly stereoselective route to anticancer compounds digitoxin and its analogues has been developed. This approach has been successfully applied to the synthesis of L-sugar digitoxin monosaccharide and several analogues. Anticancer tests showed that these analogues have excellent anticancer activities. 


\section{Chapter II}

\section{Experimental Section}

\section{General Methods and Materials.}

${ }^{1} \mathrm{H}$ and ${ }^{13} \mathrm{C}$ NMR spectra were recorded on Jeol (270 MHz) and Varian VXR-600 (600 $\mathrm{MHz}$ ) spectrometers. Chemical shifts are reported relative to internal tetramethylsilane ( $\delta$ $0.00 \mathrm{ppm})$ or $\mathrm{CDCl}_{3}(\delta 7.26 \mathrm{ppm})$ for ${ }^{1} \mathrm{H} \mathrm{NMR}$ and $\mathrm{CDCl}_{3}(\delta 77.0 \mathrm{ppm})$ for ${ }^{13} \mathrm{C} \mathrm{NMR}$. Infrared (IR) spectra were obtained on a Prospect MIDAC FT-IR spectrometer. Optical rotations were measured with a Jasco DIP-370 digital polarimeter in the solvent specified. Melting points were determined with Electrothermal Mel-Temp apparatus and are uncorrected. Flash column chromatography was performed on ICN reagent 60 (60-200 mesh) silica gel. Analytical thin-layer chromatography was performed with precoated glass-backed plates (Whatman K6F 60A, $\mathrm{F}_{254}$ ) and visualized by quenching of fluorescence and by charring after treatment with $p$-anisaldehyde or phosphomolybdic acid or potassium permanganate stain. $R_{f}$ values are obtained by elution in the stated solvent ratios $(\mathrm{v} / \mathrm{v})$. Ether, THF, methylene chloride and triethylamine were dried by passing through activated alumina ( $8 \times 14$ meshes) column with argon gas pressure. Commercial reagents were used without purification unless otherwise noted. Melting points are uncorrected. Air and/or moisture-sensitive reactions were carried out under an atmosphere of argon/nitrogen using oven/flamed-dried glassware and standard syringe/septa techniques. 


\section{(S)-1-(2-Furyl)-ethanol (19): ${ }^{14}$}

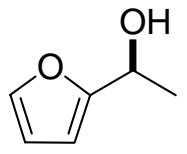

To a $25 \mathrm{ml}$ flask was added furan ketone 14 (22.0 g, $7.27 \mathrm{mmol}), \mathrm{CH}_{2} \mathrm{Cl}_{2}(101.8 \mathrm{~mL})$, formic acid/triethylamine $(1: 1,110.6 \mathrm{~mL})$ and Noyori asymmetric transfer hydrogenation catalyst (R)-Ru( $\eta^{6}$-mesitylene)-(S,S)-TsDPEN (582 $\left.\mathrm{mg}, 0.5 \mathrm{~mol} \%\right)$. The resulting solution was stirred at room temperature for $24 \mathrm{~h}$. The reaction mixture was diluted with water $(200 \mathrm{~mL})$ and extracted with EtOAc $(3 \times 500 \mathrm{ml})$. The combined organic layers were washed with saturated $\mathrm{NaHCO}_{3}$, dried over $\mathrm{Na}_{2} \mathrm{SO}_{4}$, and concentrated under reduced pressure. The crude product was purified by silica gel flash chromatography eluting with 30\% ether/hexanes to give furan alcohol 19 (21.3 g, $192 \mathrm{mmol}, 96 \%)$ : colorless oil; $\mathrm{R}_{\mathrm{f}}(30 \%$ EtOAc/hexanes $)=0.41 ;[\alpha]_{\mathrm{D}}^{25}=+21\left(\mathrm{c}=1.0, \mathrm{CH}_{2} \mathrm{Cl}_{2}\right)$; IR (thin film, $\left.\mathrm{cm}^{-1}\right) 3360,2980,2935,1668,1505,1467,1370,1229,1149,1007,877,734 ;{ }^{1} \mathrm{H}$ NMR $\left(600 \mathrm{MHz} \mathrm{CDCl}_{3}\right) \delta 7.30(\mathrm{~d}, J=1.8,1 \mathrm{H}), 6.26(\mathrm{dd}, J=3.0,1.8 \mathrm{~Hz}, 1 \mathrm{H}), 6.15(\mathrm{~d}$, $J=3.0,1 \mathrm{H}), 4.78(\mathrm{dq}, J=6.6,6.6 \mathrm{~Hz}, 1 \mathrm{H}), 3.11(\mathrm{~s}, 1 \mathrm{H}), 1.46(\mathrm{~d}, J=6.6 \mathrm{~Hz}, 3 \mathrm{H}) ;{ }^{13} \mathrm{C}$ NMR (150 MHz, $\left.\mathrm{CDCl}_{3}\right) \delta 157.7,141.6,109.9,104.9,63.3,21.1$. 
(2S)-6-Hydroxy-2-methyl-2H-Pyran-3 (6H)-one (20): ${ }^{14}$

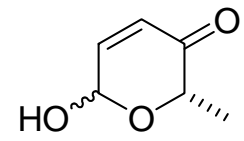

Furan alcohol 19 (21.0 g, $189 \mathrm{mmol})$, THF (466 mL), and $\mathrm{H}_{2} \mathrm{O}(155 \mathrm{~mL})$ were added to a round bottom flask and cooled to $0{ }^{\circ} \mathrm{C}$. Solid $\mathrm{NaHCO}_{3}(33.4 \mathrm{~g}, 378 \mathrm{mmol}), \mathrm{NaOAc} \cdot 3 \mathrm{H}_{2} \mathrm{O}$ (25.6 g, $189 \mathrm{mmol}$ ), and NBS (33.2 $\mathrm{g}, 189 \mathrm{mmol})$ were added to the solution and the mixture was stirred for $1 \mathrm{~h}$ at $0{ }^{\circ} \mathrm{C}$. The reaction was quenched with saturated $\mathrm{NaHCO}_{3}$ $(400 \mathrm{~mL})$, extracted $(3 \times 400 \mathrm{~mL})$ with $\mathrm{Et}_{2} \mathrm{O}$, dried $\left(\mathrm{Na}_{2} \mathrm{SO}_{4}\right)$, concentrated under reduced pressure and purified by silica gel chromatography eluting with $25 \%$ EtOAc/hexanes to give pyranone $20(21.8 \mathrm{~g}, 172 \mathrm{mmol}, 91 \%): \mathrm{R}_{\mathrm{f}}(60 \%$ EtOAc/hexanes $)=0.29 ;[\alpha]^{25}=+$ $44\left(\mathrm{c}=1.0, \mathrm{CH}_{2} \mathrm{Cl}_{2}\right.$ ); IR (thin film, $\mathrm{cm}^{-1}$ ) 3381, 2988, 2942, 1692, 1447, 1373, 1232, 1021, 937; ${ }^{1} \mathrm{H}$ NMR (600 MHz, $\left.\mathrm{CDCl}_{3}\right)$ major isomer $\delta 6.82(\mathrm{dd}, J=10.2,3.0 \mathrm{~Hz}, 1 \mathrm{H})$, $5.96(\mathrm{~d}, J=10.2,1 \mathrm{H}), 5.48(\mathrm{~d}, J=3.0 \mathrm{~Hz}, 1 \mathrm{H}), 3.99(\mathrm{q}, J=7.2 \mathrm{~Hz}, 1 \mathrm{H}), 1.23(\mathrm{~d}, J=7.2$ $\mathrm{Hz}, 3 \mathrm{H}) ;{ }^{13} \mathrm{C} \mathrm{NMR}\left(150 \mathrm{MHz}, \mathrm{CDCl}_{3}\right)$ major isomer $\delta 197.6,145.3,126.6,87.2,74.8$, 15.1 . 
(2S, 6S)-tert-butyl -5,6-dihydro-6-methyl-5-oxo-2H-pyran-2-yl carbonate (18): ${ }^{14}$<smiles>C[C@@H]1O[C@@H](OC(C)(C)C)C=CC1=O</smiles>

A benzene solution $(288 \mathrm{~mL})$ of pyronone alcohol 20 (15.1 g, $118 \mathrm{mmol})$ was added sodium acetate $(10.7 \mathrm{~g}, 130 \mathrm{mmol})$ and $(\mathrm{Boc})_{2} \mathrm{O}(38.7 \mathrm{~g}, 117 \mathrm{mmol})$. The mixture was heated to $80{ }^{\circ} \mathrm{C}$ for $1 \mathrm{~h}$. Then it was cooled down and quenched with $200 \mathrm{~mL}$ ice cold saturated $\mathrm{NaHCO}_{3}$, extracted with $\mathrm{Et}_{2} \mathrm{O}(3 \times 500 \mathrm{~mL})$, dried $\left(\mathrm{Na}_{2} \mathrm{SO}_{4}\right)$, and concentrated under reduced pressure. The crude product was purified using silica gel flash chromatography eluting with 6\% EtOAc/hexanes to give $22.0 \mathrm{~g}(96.8 \mathrm{mmol}, 82 \%)$ of two diastereomers of Boc-protected pyranone $18 \alpha$ and $18 \beta$ in $1: 1.3: \mathrm{R}_{\mathrm{f}}\left(20 \% \mathrm{Et}_{2} \mathrm{O} /\right.$ hexane $)=$ $0.58 ;[\alpha]_{D}^{25}=+98\left(\mathrm{c}=1.0, \mathrm{CH}_{2} \mathrm{Cl}_{2}\right) ;$ IR (thin film, $\left.\mathrm{cm}^{-1}\right) 2984,2942,1752,1703,1371$, 1273, 1254, 1153, 938, 838; ${ }^{1} \mathrm{H}$ NMR $\left(600 \mathrm{MHz}, \mathrm{CDCl}_{3}\right) \delta 6.78(\mathrm{dd}, J=10.2,3.6 \mathrm{~Hz}$, 1H), $6.22(\mathrm{~d}, J=3.6 \mathrm{~Hz}, 1 \mathrm{H}), 6.09(\mathrm{~d}, J=10.2 \mathrm{~Hz}, 1 \mathrm{H}), 4.53$ (q, $J=6.6 \mathrm{~Hz}, 1 \mathrm{H}), 1.40$ (s, 9H), $1.28(\mathrm{~d}, J=6.6 \mathrm{~Hz}, 3 \mathrm{H}) ;{ }^{13} \mathrm{C} \mathrm{NMR}\left(150 \mathrm{MHz}, \mathrm{CDCl}_{3}\right) \delta 195.5,151.7,140.9,128.2$, 89.1, 83.3, 72.0, 27.5, 15.1; ClHRMS Calculated for $\left[\mathrm{C}_{11} \mathrm{H}_{16} \mathrm{O}_{5} \mathrm{Na}^{+}\right]$: 251.0890, Found: 251.0883 


\section{(2S, 6S)-2-Digitoxigenoxy-6-methyl-2H-pyran-3(6H)-one (23):}

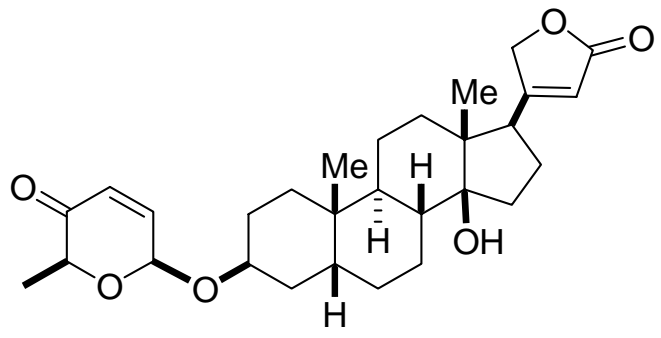

$\mathrm{A} \mathrm{CH}_{2} \mathrm{Cl}_{2} /$ THF solution (19 mL, 4:1 V/V) of Boc-pyranone $\mathbf{1 8 \beta}(1.29 \mathrm{~g}, 5.65 \mathrm{mmol})$ and digitoxigenin $(3.17 \mathrm{~g}, 8.48 \mathrm{mmol})$ was cooled to $0{ }^{\circ} \mathrm{C}$. $\mathrm{A} \mathrm{CH}_{2} \mathrm{Cl}_{2}(2.5 \mathrm{~mL})$ solution of $\mathrm{Pd}_{2}(\mathrm{DBA})_{3} \cdot \mathrm{CHCl}_{3}(170 \mathrm{mg}, 2.5 \mathrm{~mol} \%)$ and $\mathrm{PPh}_{3}(171 \mathrm{mg}, 10 \mathrm{~mol} \%)$ was added to the reaction mixture at $0{ }^{\circ} \mathrm{C}$. The reaction mixture was stirred at $0{ }^{\circ} \mathrm{C}$ for 8 hours and was quenched with $20 \mathrm{~mL}$ of satd. aq $\mathrm{NaHCO}_{3}$, extracted $(3 \times 50 \mathrm{~mL})$ with $\mathrm{Et}_{2} \mathrm{O}$, dried $\left(\mathrm{Na}_{2} \mathrm{SO}_{4}\right)$, and concentrated under reduced pressure. The crude product was purified using silica gel flash chromatography eluting with 40\% EtOAc/hexanes to give 23 (2.32 g, $4.80 \mathrm{mmol}, 85 \%)$ as a white solid: $\mathrm{R}_{\mathrm{f}}(40 \%$ EtOAc/hexanes $)=0.17 ; \mathrm{mp}: 211-212{ }^{\circ} \mathrm{C}$; $[\alpha]^{25}=+5.28\left(\mathrm{c}=1.0, \mathrm{CHCl}_{3}\right) ;$ IR (thin film, $\left.\mathrm{cm}^{-1}\right) 3505,2938,2875,2376,2311,1780$, $1741,1698,1620,1448,1374,1164,1144,1053,1028,730 ;{ }^{1} \mathrm{H} \mathrm{NMR}\left(600 \mathrm{MHz}, \mathrm{CDCl}_{3}\right)$ $\delta 6.87(\mathrm{dd}, \mathrm{J}=10.4,1.8 \mathrm{~Hz}, 1 \mathrm{H}), 6.12(\mathrm{dd}, \mathrm{J}=10.2,1.2 \mathrm{~Hz}, 1 \mathrm{H}), 5.87$ (m, 1H), 5.39 (dd, $J=2.4,1.8 \mathrm{~Hz}, 1 \mathrm{H}), 4.99(\mathrm{dd}, J=18.0,1.8 \mathrm{~Hz}, 1 \mathrm{H}), 4.81(\mathrm{dd}, J=18.0,1.8 \mathrm{~Hz}, 1 \mathrm{H}), 4.18$ (m, 2H), $2.78(\mathrm{dd}, J=9.6,6.0 \mathrm{~Hz}, 1 \mathrm{H}), 2.20-2.09(\mathrm{~m}, 3 \mathrm{H}), 1.45(\mathrm{~d}, J=6.6 \mathrm{~Hz}, 3 \mathrm{H}), 1.92-$ $1.16(\mathrm{~m}, 18 \mathrm{H}), 0.95(\mathrm{~s}, 3 \mathrm{H}), 0.88(\mathrm{~s}, 3 \mathrm{H}) ;{ }^{13} \mathrm{C} \mathrm{NMR}\left(150 \mathrm{MHz}, \mathrm{CDCl}_{3}\right) \delta$ 197.0, 174.4, $174.3,147.7,128.2,117.8,93.9,85.6,75.2,73.4,73.3,50.9,49.6,41.9,40.0,36.3,35.8$, $35.2,33.2,32.0,30.0,26.9,26.4,24.4,23.7,21.3,21.2,17.0,15.8$; ESIHRMS Calcd for $\left[\mathrm{C}_{29} \mathrm{H}_{40} \mathrm{O}_{6} \mathrm{Na}^{+}\right]:$507.2717, Found 507.2722. 


\section{(2S, 6S)-2-(Digitoxigenoxy)-2,5-dihydro-6-methyl-2H-pyran-5-ol (24):}

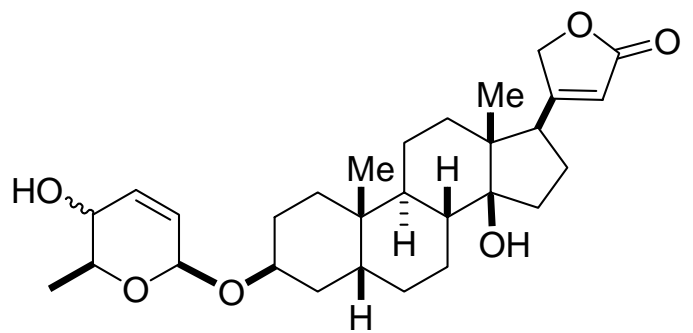

A $\mathrm{CH}_{2} \mathrm{Cl}_{2}(9.4 \mathrm{~mL})$ solution of enone $23(2.32 \mathrm{~g}, 4.80 \mathrm{mmol})$ and $\mathrm{CeCl}_{3}$ in $\mathrm{MeOH}$ solution $(0.4 \mathrm{M}, 9.4 \mathrm{~mL})$ was cooled to $-78{ }^{\circ} \mathrm{C} . \mathrm{NaBH}_{4}(162 \mathrm{mg}, 4.80 \mathrm{mmol})$ was added and the reaction mixture was stirred at $-78{ }^{\circ} \mathrm{C}$ for $3 \mathrm{~h}$. The reaction mixture was diluted with ether $(60 \mathrm{~mL})$ and was quenched with $30 \mathrm{~mL}$ of satd. aq $\mathrm{NaHCO}_{3}$, extracted $(3 \times 60$ $\mathrm{mL})$ with $\mathrm{Et}_{2} \mathrm{O}$, dried $\left(\mathrm{Na}_{2} \mathrm{SO}_{4}\right)$, and concentrated under reduced pressure. The crude product was purified using silica gel flash chromatography eluting with 55\% EtOAc/hexanes to give allylic alcohols $\mathbf{2 4 a} / \mathbf{b}(1.89 \mathrm{~g}, 3.88 \mathrm{mmol}, 81 \%)$ as a white solid (diastereometric ratio $\mathbf{2 4 a : 2 4 b}=1.5: 1$, inseparable by silica gel chromatography): $\mathrm{R}_{\mathrm{f}}$ $(60 \%$ EtOAc/hexanes $)=0.22 ;$ IR $\left(\right.$ thin film, $\left.\mathrm{cm}^{-1}\right)$ 3449, 2934, 2871, 1779, 1737, 1619, 1448, 1380, 1320, 1169, 1136, 1051, 1026, 1006, 961, 751; ${ }^{1} \mathrm{H}$ NMR (600 MHz, $\left.\mathrm{CDCl}_{3}\right)$ 24a $\delta 6.12(\mathrm{ddd}, J=10.2,4.8,1.2 \mathrm{~Hz}, 1 \mathrm{H}), 5.86(\mathrm{~m}, 1 \mathrm{H}), 5.79(\mathrm{~d}, J=10.2 \mathrm{~Hz}, 1 \mathrm{H}), 5.07$ (m, 1H), $4.97(\mathrm{dd}, J=18.0,1.2 \mathrm{~Hz}, 1 \mathrm{H}), 4.80(\mathrm{dd}, J=18.0,1.8 \mathrm{~Hz}, 1 \mathrm{H}), 4.12(\mathrm{dd}, J=4.2$, $1.8 \mathrm{~Hz}, 1 \mathrm{H}), 4.11(\mathrm{~s}, 1 \mathrm{H}), 3.70(\mathrm{qd}, J=6.6,2.4 \mathrm{~Hz}, 1 \mathrm{H}), 3.60(\mathrm{br}, 1 \mathrm{H}), 2.77(\mathrm{dd}, J=9.6$, $6.0 \mathrm{~Hz}, 1 \mathrm{H}), 2.25-2.05(\mathrm{~m}, 3 \mathrm{H}), 1.30(\mathrm{~d}, J=6.0 \mathrm{~Hz}, 3 \mathrm{H}), 1.80-1.05(\mathrm{~m}, 18 \mathrm{H}), 0.93(\mathrm{~s}$, 3H), $0.86(\mathrm{~s}, 3 \mathrm{H}) ; 24 \mathbf{b} \delta 5.91\left(\mathrm{ddd}_{2} J=10.2,2.4,2.4 \mathrm{~Hz}, 1 \mathrm{H}\right), 5.86(\mathrm{~m}, 1 \mathrm{H}), 5.74(\mathrm{ddd}, J$ $=10.2,1.2,1.2 \mathrm{~Hz}, 1 \mathrm{H}), 5.13(\mathrm{ddd}, J=1.8,1.8,1.8 \mathrm{~Hz}, 1 \mathrm{H}), 4.98(\mathrm{dd}, J=18.0,1.2 \mathrm{~Hz}$, 1H), $4.80(\mathrm{dd}, J=18.0,1.2 \mathrm{~Hz}, 1 \mathrm{H}), 4.09(\mathrm{~m}, 1 \mathrm{H}), 4.11(\mathrm{~s}, 1 \mathrm{H}), 3.91(\mathrm{br}, 1 \mathrm{H}), 3.59(\mathrm{dq}, J$ $=6.6,6.6 \mathrm{~Hz}, 1 \mathrm{H}), 2.77(\mathrm{dd}, J=9.6,6.0 \mathrm{~Hz}, 1 \mathrm{H}), 2.25-2.05(\mathrm{~m}, 3 \mathrm{H}), 1.33(\mathrm{~d}, J=6.0 \mathrm{~Hz}$, 
3H), 1.80-1.05 (m, 18H), 0.94 (s, 3H), 0.87 (s, 3H); $\left.{ }^{13} \mathrm{C} \mathrm{NMR} \mathrm{(150} \mathrm{MHz,} \mathrm{CDCl}_{3}\right)$ 24a $\delta$ 174.64, 174.60, 131.8, 131.0, 117.6, 96.0, 85.3, 74.3, 73.1, 71.4, 64.6, 50.9, 49.6, 41.8, $40.0,36.4,35.7,35.1,33.0,32.1,29.9,26.9$ (2C), 26.4, 23.6, 21.2, 21.1, 16.7, 15.7; 24b $\delta$ 174.67, 174.60, 131.3, 129.8, 117.6, 94.8, 85.4, 73.4, 73.1, 72.3, 68.5, 50.9, 49.6, 41.8, $40.0,36.3,35.7,35.1,33.1,32.0,29.9,26.8$ (2C), 26.6, 23.6, 21.2, 21.1, 18.3, 15.7; ESIHRMS Calcd for $\left[\mathrm{C}_{29} \mathrm{H}_{42} \mathrm{O}_{6} \mathrm{Na}^{+}\right]: 509.2879$, Found 509.2879. 


\section{(2S, 6S)-2-(Digitoxigenoxy)-3,6-dihydro-6-methyl-2H-pyran (25):}

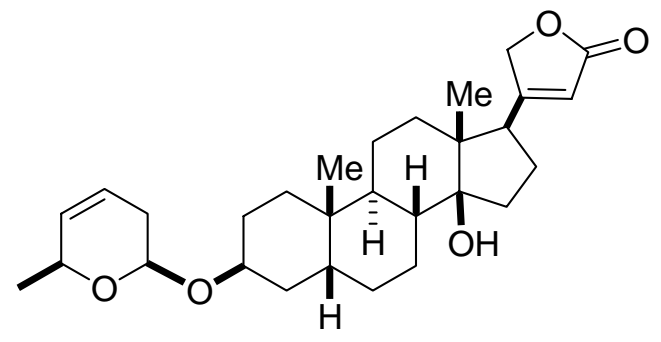

A flask was charged with dry $N$-methyl morpholine (NMM) $5.76 \mathrm{~mL}$, triphenyl phosphine (3.36 $\mathrm{g}, 12.8 \mathrm{mmol})$ and was cooled to $-30{ }^{\circ} \mathrm{C}$ under $\mathrm{Ar}$ atmosphere. Diethylazodicarboxylate $(1.83 \mathrm{~mL}, 11.7 \mathrm{mmol})$ was added and the reaction was stirred for $5 \mathrm{~min}$, allylic alcohol $\mathbf{2 4 a} / \mathbf{b}(1.89 \mathrm{~g}, 3.88 \mathrm{mmol})$ was added in a $1 \mathrm{M}$ solution of NMM and the reaction mixture was stirred for $10 \mathrm{~min}$, followed by addition of $o$ nitrobenzenesulfonyl hydrazide (NBSH) $(2.36 \mathrm{~g}, 12.8 \mathrm{mmol})$. The reaction was stirred at $-30{ }^{\circ} \mathrm{C}$ for $6 \mathrm{~h}$ and was monitored by TLC, upon consumption of starting material, warm up to room temperature and stirred for another $1 \mathrm{~h}$. The reaction mixture was diluted with ether $(60 \mathrm{~mL})$ and was quenched with $60 \mathrm{~mL}$ of satd. aq $\mathrm{NaHCO}_{3}$, extracted $(3 \times 60 \mathrm{~mL})$ with $\mathrm{Et}_{2} \mathrm{O}$, dried $\left(\mathrm{Na}_{2} \mathrm{SO}_{4}\right)$, and concentrated under reduced pressure. The crude product was purified using silica gel flash chromatography eluting with $25 \%$ EtOAc/hexanes to give product $25(1.80 \mathrm{~g}, 3.82 \mathrm{mmol}, 98 \%)$ as a white solid: $\mathrm{R}_{\mathrm{f}}(30 \%$ EtOAc/hexanes $)=$ 0.20; mp: $157-158^{\circ} \mathrm{C} ;[\alpha]^{25}=+23.3\left(\mathrm{c}=1.1, \mathrm{CHCl}_{3}\right)$; IR (thin film, $\left.\mathrm{cm}^{-1}\right) 3301,2933$, 2871, 1778, 1742, 1620, 1447, 1378, 1221, 1157, 1133, 1097, 1065, 1024, 974, 909, 782. ${ }^{1} \mathrm{H}$ NMR $\left(600 \mathrm{MHz}, \mathrm{CDCl}_{3}\right) \delta 5.86(\mathrm{~m}, 1 \mathrm{H}), 5.66(\mathrm{dddd}, J=10.2,4.8,2.4,2.4 \mathrm{~Hz}, 1 \mathrm{H})$, $5.55(\mathrm{dddd}, J=10.2,2.4,1.2,1.2 \mathrm{~Hz}, 1 \mathrm{H}), 4.99(\mathrm{dd}, J=18.0,1.2 \mathrm{~Hz}, 1 \mathrm{H}), 4.80$ (dd, $J=$ 18.0, 1.2 Hz, 1H), $4.70(\mathrm{dd}, J=8.4,3.0 \mathrm{~Hz}, 1 \mathrm{H}), 4.06(\mathrm{~m}, 1 \mathrm{H}), 4.29(\mathrm{~m}, 1 \mathrm{H}), 2.76(\mathrm{~m}$, 1H), 2.24-2.04 (m, 4H), 1.90-1.08 (m, 19H), $1.24(\mathrm{~d}, J=6.0 \mathrm{~Hz}, 3 \mathrm{H}), 0.92(\mathrm{~s}, 3 \mathrm{H}), 0.86$ 
(s, 3H); ${ }^{13} \mathrm{C}$ NMR $\left(150 \mathrm{MHz}, \mathrm{CDCl}_{3}\right) \delta 174.6,174.5,131.1,123.0,117.6,96.7,85.6$, $73.4,72.1,70.7,50.9,49.6,41.9,40.1,36.3,35.7,35.2,33.1,31.6,30.2,29.8,26.9,26.7$, 26.6, 23.6, 21.4, 21.1, 21.0, 15.8; ESIHRMS Calcd for $\left[\mathrm{C}_{29} \mathrm{H}_{42} \mathrm{O}_{5} \mathrm{Na}^{+}\right]$: 493.2929, Found 493.2930. 


\section{Digitoxigen 2,6-dideoxy- $\beta$-L-ribo-hexopyranoside (15):}

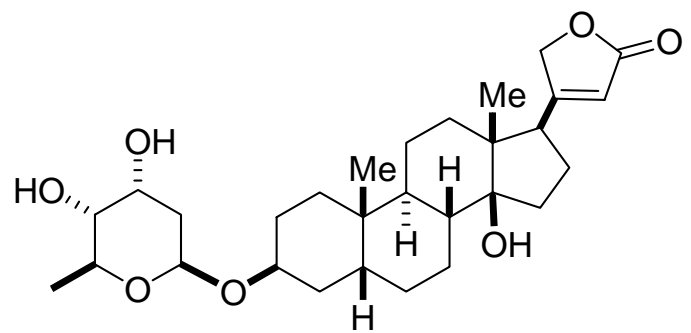

To a $t-\mathrm{BuOH} /$ acetone $(4 \mathrm{~mL})$ solution of olefin $25(1.80 \mathrm{~g}, 3.82 \mathrm{mmol})$ at $0{ }^{\circ} \mathrm{C}$ was added a solution of $(50 \% \mathrm{w} / \mathrm{v})$ of $\mathrm{N}$-methyl morpholine $\mathrm{N}$-oxide/water $(2.4 \mathrm{~mL})$. Crystalline $\mathrm{OsO}_{4}(9.6 \mathrm{mg}, 1 \mathrm{~mol} \%)$ was added and the reaction was stirred for $4 \mathrm{~h}$. The reaction was quenched with adding EtOAc and satd. $\mathrm{NaHCO}_{3}$. The organic layer was separated and concentrated. It was purified by a silica gel column using 90\% EtOAc/hexanes. Pure fractions were combined, concentrated, and crystallized from acetone/hexanes to afford alcohol $15(2.07 \mathrm{~g}, 3.55 \mathrm{mmol}, 93 \%)$ as a white solid: $\mathrm{R}_{\mathrm{f}}($ EtOAc $)=0.42 ;[\alpha]^{25}=+30.2$ $(\mathrm{c}=1.0, \mathrm{MeOH}) ; \mathrm{mp}: 188-189^{\circ} \mathrm{C}$; IR (thin film, $\mathrm{cm}^{-1}$ ) 3440, 2934, 2856, 2193, 1736, 1619, 1448, 1380, 1160, 1134, 1065, 1026,1002, 949, 906, 824; ${ }^{1} \mathrm{H}$ NMR $(600 \mathrm{MHz}$ $\left.\mathrm{CDCl}_{3}\right) \delta 5.86(\mathrm{~m}, 1 \mathrm{H}), 4.98(\mathrm{~d}, J=18.0 \mathrm{~Hz}, 1 \mathrm{H}), 4.86(\mathrm{dd}, J=9.0,1.8 \mathrm{~Hz}, 1 \mathrm{H}), 4.80(\mathrm{~d}$, $J=18.0 \mathrm{~Hz}, 1 \mathrm{H}), 4.11(\mathrm{ddd}, J=3.0,3.0,3.0 \mathrm{~Hz}, 1 \mathrm{H}), 4.02(\mathrm{~m}, 1 \mathrm{H}), 3.71(\mathrm{dq}, J=9.0,6.0$ $\mathrm{Hz}, 1 \mathrm{H}), 3.32(\mathrm{~m}, 1 \mathrm{H}), 2.77(\mathrm{~m}, 1 \mathrm{H}), 2.56(\mathrm{~s}, 1 \mathrm{H}), 2.39(\mathrm{~s}, 1 \mathrm{H}), 2.20-2.00(\mathrm{~m}, 4 \mathrm{H}), 1.29$ $(\mathrm{d}, J=6.0 \mathrm{~Hz}, 3 \mathrm{H}), 1.90-1.10(\mathrm{~m}, 19 \mathrm{H}), 0.91(\mathrm{~s}, 3 \mathrm{H}), 0.86(\mathrm{~s}, 3 \mathrm{H}) ;{ }^{13} \mathrm{C}$ NMR $(150 \mathrm{MHz}$, $\left.\mathrm{CDCl}_{3}\right) \delta 175.0,174.9,117.8,95.6,85.8,73.8,73.2,72.9,69.5,68.5,51.2,49.8,42.0$ $40.2,38.5,36.5,35.9,35.4,33.4,32.3,30.2,27.1,26.6,24.6,23.8,21.5,21.4,18.4,16.0$; ESIHRMS Calcd for $\left[\mathrm{C}_{29} \mathrm{H}_{44} \mathrm{O}_{7} \mathrm{Na}^{+}\right]$: 527.2979, Found 527.2985. 
(2S, 6S)-2-methyl-3-hydroxy-6-Digitoxigenoxy-2H-pyran-4-nitrobenzoate (30):

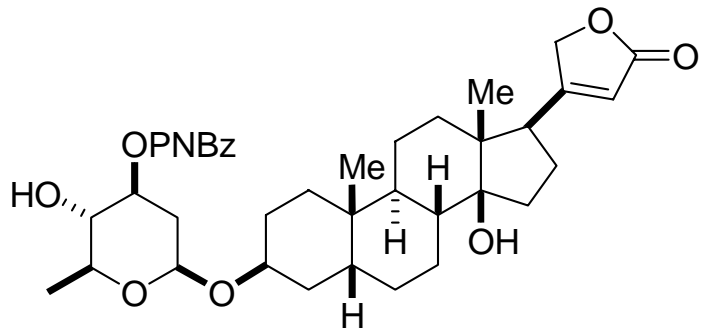

A THF $(0.6 \mathrm{~mL})$ solution of diol $15(50 \mathrm{mg}, 0.1 \mathrm{mmol})$ at $0{ }^{\circ} \mathrm{C}$ was added $\mathrm{PPh}_{3}(41 \mathrm{mg}$, $0.16 \mathrm{mmol})$ and $p$-nitrobenzoic acid (40 mg, $0.2 \mathrm{mmol})$, DEAD (27.5 $\mathrm{mg}, 0.16 \mathrm{mmol})$ in THF $(0.2 \mathrm{~mL})$ was added drop wise. The reaction mixture was warmed up to room temperature and stirred for $5 \mathrm{~h}$. The reaction mixture was diluted with $5 \mathrm{~mL}$ EtOAc and quenched with $4 \mathrm{~mL}$ of satd. $\mathrm{NaHCO}_{3}$, extracted with $\mathrm{Et}_{2} \mathrm{O}(3 \times 5 \mathrm{~mL})$, dried $\left(\mathrm{Na}_{2} \mathrm{SO}_{4}\right)$, and concentrated under reduced pressure. The crude product was purified using silica gel flash chromatography eluting with 60\% EtOAc/hexanes to afford 30 (56 mg, $0.85 \mathrm{mmol}$, $85 \%)$ as a white solid: $\mathrm{R}_{\mathrm{f}}(\mathrm{EtOAc})=0.75 ;[\alpha]_{\mathrm{D}}^{25}=+8.68\left(\mathrm{c}=0.5, \mathrm{CHCl}_{3}\right) ; \mathrm{mp}: 188-189$ ${ }^{\circ} \mathrm{C}$; IR (thin film, $\mathrm{cm}^{-1}$ ) 3484, 2931, 2364, 2197, 2168, 2038, 1730, 1529, 1448, 1346, 1278, 1103, 1069, 1026, 989, 908; ${ }^{1} \mathrm{H}$ NMR (600 MHz, $\left.\mathrm{CDCl}_{3}\right) \delta 8.25(\mathrm{~m}, 4 \mathrm{H}), 5.86(\mathrm{~m}$, 1H), $5.10(\mathrm{~m}, 1 \mathrm{H}), 4.98(\mathrm{~d}, J=18.0 \mathrm{~Hz}, 1 \mathrm{H}), 4.80(\mathrm{~d}, J=18.0 \mathrm{~Hz}, 1 \mathrm{H}), 4.66(\mathrm{dd}, J=8.4$, $1.2 \mathrm{~Hz}, 1 \mathrm{H}), 4.07(\mathrm{~m}, 1 \mathrm{H}), 3.48(\mathrm{~m}, 1 \mathrm{H}), 3.40(\mathrm{dq}, J=9.0,6.0 \mathrm{~Hz}, 1 \mathrm{H}), 2.77(\mathrm{~m}, 1 \mathrm{H})$, $2.39(\mathrm{dd}, J=12.0,6.0 \mathrm{~Hz}, 1 \mathrm{H}), 2.20-2.05(\mathrm{~m}, 4 \mathrm{H}), 1.39$ (d, $J=6.0 \mathrm{~Hz}, 3 \mathrm{H}), 1.90-1.10$ (m, 19H), $0.93(\mathrm{~s}, 1 \mathrm{H}), 0.91(\mathrm{~s}, 3 \mathrm{H}), 0.86(\mathrm{~s}, 3 \mathrm{H}) ;{ }^{13} \mathrm{C} \mathrm{NMR}\left(150 \mathrm{MHz}, \mathrm{CDCl}_{3}\right) \delta 174.5$, $174.45,131.1,123.0,117.7,96.7(2 \mathrm{C}), 85.6,73.4,73.1,72.1,70.7,62.2(2 \mathrm{C}), 51.0,50.9$, $49.6,41.9,40.1,36.3,35.7,35.2,33.2,32.2,31.6,30.0,26.9,26.4,24.3,23.6,21.3,21.2$, 21.1, 15.8, 14.4 (2C); ESIHRMS Calcd for $\left[\mathrm{C}_{36} \mathrm{H}_{47} \mathrm{NO}_{10} \mathrm{Na}^{+}\right]$: 653.7591, Found 653.7591. 


\section{Digitoxigen 2,6-dideoxy- $\beta$-L-gluco-hexopyranoside (26):}

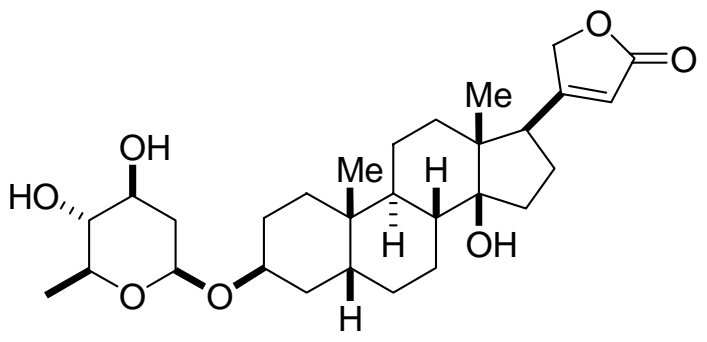

$\mathrm{A} \mathrm{MeOH}(0.2 \mathrm{~mL})$ solution of $\mathbf{3 0}(28 \mathrm{mg}, 42 \mu \mathrm{mol})$ at room temperature was added $\mathrm{Et}_{3} \mathrm{~N}$ (12.6 mg, $126 \mu \mathrm{mol})$ and the reaction mixture was stirred for $2 \mathrm{~h}$. The reaction mixture was diluted with $5 \mathrm{~mL}$ EtOAc and quenched with $4 \mathrm{~mL}$ of Satd. $\mathrm{NaHCO}_{3}$, extracted $(3 \times 5$ $\mathrm{mL}$ ) with $\mathrm{Et}_{2} \mathrm{O}$, dried $\left(\mathrm{Na}_{2} \mathrm{SO}_{4}\right)$, and concentrated under reduced pressure. It was purified by a silica gel column using 90\% EtOAc/hexanes. Pure fractions were combined, concentrated, and crystallized from acetone/hexanes to afford $\mathbf{2 6}(20.1 \mathrm{mg}, 40 \mu \mathrm{mol}, 95 \%)$ as a white solid: $\mathrm{R}_{\mathrm{f}}($ EtOAc $)=0.42 ;[\alpha]^{25}=+30.2(\mathrm{c}=1.0, \mathrm{MeOH}) ; \mathrm{mp}: 255-256{ }^{\circ} \mathrm{C}$; IR (thin film, $\mathrm{cm}^{-1}$ ) 3453, 2940, 2856, 2173, 1969, 1775, 1742, 1623, 1449, 1454, 1378, 1160, 1067, 1024, 951, 822; ${ }^{1} \mathrm{H}$ NMR (600 MHz, $\left.\mathrm{CDCl}_{3}\right) \delta 5.86(\mathrm{~m}, 1 \mathrm{H}), 4.98(\mathrm{dd}, J=$ 18.0, $1.8 \mathrm{~Hz}, 1 \mathrm{H}), 4.80(\mathrm{dd}, J=18.0,1.8 \mathrm{~Hz}, 1 \mathrm{H}), 4.53(\mathrm{dd}, J=9.6,1.8 \mathrm{~Hz}, 1 \mathrm{H}), 4.02(\mathrm{~m}$, 1H), $3.58(\mathrm{dq}, J=9.0,6.0 \mathrm{~Hz}, 1 \mathrm{H}), 3.24(\mathrm{~m}, 1 \mathrm{H}), 3.09(\mathrm{~m}, 1 \mathrm{H}), 2.77(\mathrm{~m}, 1 \mathrm{H}), 2.33(\mathrm{~s}$, 1H), 2.20-2.00 (m, 4H), $1.26(\mathrm{~d}, J=6.0 \mathrm{~Hz}, 3 \mathrm{H}), 1.90-1.10(\mathrm{~m}, 21 \mathrm{H}), 0.92(\mathrm{~s}, 3 \mathrm{H}), 0.86$ $(\mathrm{s}, 3 \mathrm{H}) ;{ }^{13} \mathrm{C} \mathrm{NMR}\left(150 \mathrm{MHz}, \mathrm{CDCl}_{3}\right) \delta 174.65,174.61,117.6,97.3,85.4,77.5,73.5$, $72.9,71.8,71.6,50.9,49.6,41.8,40.0,39.5,36.2,35.7,35.1,33.1,32.0,29.9,26.9,26.4$, 24.4, 23.6, 21.2, 21.1, 17.8, 15.8; ESIHRMS Calcd for $\left[\mathrm{C}_{29} \mathrm{H}_{44} \mathrm{O}_{7} \mathrm{Na}^{+}\right]$: 527.2979, Found 527.2985 


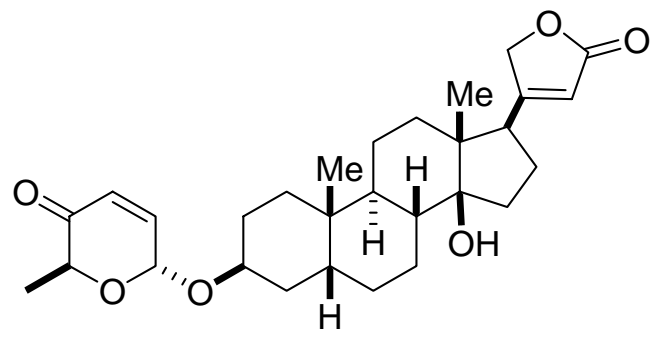

$\mathrm{A} \mathrm{CH}_{2} \mathrm{Cl}_{2} / \mathrm{THF}$ solution $(1.5 \mathrm{~mL}, 4: 1 \mathrm{~V} / \mathrm{V})$ of Boc-pyranone $\mathbf{1 8 \alpha}(316 \mathrm{mg}, 1.39 \mathrm{mmol})$ and digitoxigenin $(260 \mathrm{mg}, 0.69 \mathrm{mmol})$ was cooled to $0{ }^{\circ} \mathrm{C}$. $\mathrm{A} \mathrm{CH}_{2} \mathrm{Cl}_{2}(0.24 \mathrm{~mL})$ solution of $\mathrm{Pd}_{2}(\mathrm{DBA})_{3} \cdot \mathrm{CHCl}_{3}(17 \mathrm{mg}, 2.5 \mathrm{~mol} \%)$ and $\mathrm{PPh}_{3}(17 \mathrm{mg}, 10 \mathrm{~mol} \%)$ was added to the reaction mixture at $0{ }^{\circ} \mathrm{C}$. The reaction mixture was stirred at $0{ }^{\circ} \mathrm{C}$ for 8 hours and was quenched with $4 \mathrm{~mL}$ of satd aq $\mathrm{NaHCO}_{3}$, extracted $(3 \times 10 \mathrm{~mL})$ with $\mathrm{Et}_{2} \mathrm{O}$, dried $\left(\mathrm{Na}_{2} \mathrm{SO}_{4}\right)$, and concentrated under reduced pressure. The crude product was purified using silica gel flash chromatography eluting with 40\% EtOAc/hexanes to give 31 (287 $\mathrm{mg}, 0.59 \mathrm{mmol}, 86 \%)$ as a white solid: $\mathrm{R}_{\mathrm{f}}(40 \%$ EtOAc/hexanes $)=0.17 ; \mathrm{mp}: 145-146{ }^{\circ} \mathrm{C}$; $[\alpha]^{25}=+61.4(\mathrm{c}=1.0, \mathrm{MeOH}) ; \mathrm{IR}\left(\right.$ thin film, $\left.\mathrm{cm}^{-1}\right)$ 3481, 2939, 2253, 1738, 1698, 1620, 1448, 1374, 1319, 1237, 1157, 1102, 1079, 1024, 958, 905, 859, 645; ${ }^{1} \mathrm{H}$ NMR (600 MHz, $\left.\mathrm{CDCl}_{3}\right) \delta 6.78(\mathrm{dd}, J=10.4,1.8 \mathrm{~Hz}, 1 \mathrm{H}), 5.99(\mathrm{dd}, J=10.2,1.2 \mathrm{~Hz}, 1 \mathrm{H}), 5.80(\mathrm{~m}, 1 \mathrm{H})$, $5.21(\mathrm{dd}, J=2.4,1.8 \mathrm{~Hz}, 1 \mathrm{H}), 4.95(\mathrm{dd}, J=18.0,1.8 \mathrm{~Hz}, 1 \mathrm{H}), 4.50(\mathrm{dd}, J=18.0,1.8 \mathrm{~Hz}$, 1H), $4.04(\mathrm{~m}, 2 \mathrm{H}), 2.73(\mathrm{dd}, J=9.6,6.0 \mathrm{~Hz}, 1 \mathrm{H}), 2.20-2.09(\mathrm{~m}, 3 \mathrm{H}), 1.30(\mathrm{~d}, J=6.6 \mathrm{~Hz}$, 3H), 1.92-1.16 (m, 18H), $0.88(\mathrm{~s}, 3 \mathrm{H}), 0.80(\mathrm{~s}, 3 \mathrm{H}) ;{ }^{13} \mathrm{C} \mathrm{NMR}\left(150 \mathrm{MHz}, \mathrm{CDCl}_{3}\right) \delta 197.2$, $174.9,174.6,144.4,126.7,117.3,91.9,85.2,75.2,74.1,73.4,50.9,49.6,41.5,40.0,36.3$, $35.5,35.0,32.8,30.3,30.1,26.8,26.4,26.3,23.6,21.2,21.0,15.6,15.0$; ESIHRMS Calcd for $\left[\mathrm{C}_{29} \mathrm{H}_{40} \mathrm{O}_{6} \mathrm{Na}^{+}\right]$: 507.2717, Found 507.2722. 


\section{Digitoxigen 2,3-dideoxy-a-L-rhamno-hexopyranoside (27):}

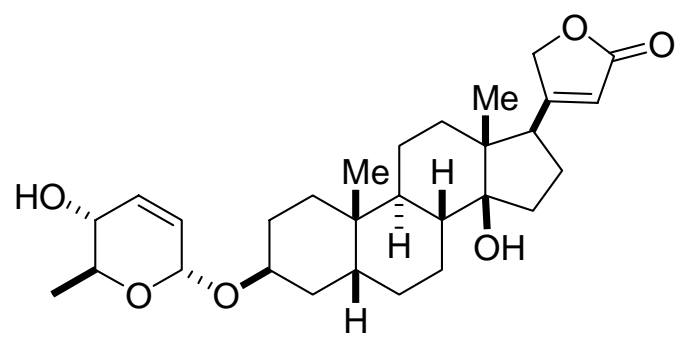

A $\mathrm{CH}_{2} \mathrm{Cl}_{2}(1.4 \mathrm{~mL})$ solution of enone $31(287 \mathrm{mg}, 0.59 \mathrm{mmol})$ and $\mathrm{CeCl}_{3}$ in $\mathrm{MeOH}$ solution $(0.4 \mathrm{M}, 1.4 \mathrm{~mL})$ was cooled to $-78{ }^{\circ} \mathrm{C} . \mathrm{NaBH}_{4}(20 \mathrm{mg}, 0.6 \mathrm{mmol})$ was added and the reaction mixture was stirred at $-78^{\circ} \mathrm{C}$ for $3 \mathrm{~h}$. The reaction mixture was diluted with ether $(10 \mathrm{~mL})$ and was quenched with $10 \mathrm{~mL}$ of satd. aq $\mathrm{NaHCO}_{3}$, extracted $(3 \times 10 \mathrm{~mL})$ with $\mathrm{Et}_{2} \mathrm{O}$, dried $\left(\mathrm{Na}_{2} \mathrm{SO}_{4}\right)$, and concentrated under reduced pressure. The crude product was purified using silica gel flash chromatography eluting with 55\% EtOAc/hexanes to give allylic alcohol $27(239 \mathrm{mg}, 0.49 \mathrm{mmol}, 83 \%)$ as a white solid: $\mathrm{R}_{\mathrm{f}}(60 \%$ EtOAc/hexanes) $=0.22$; mp: $193-194{ }^{\circ} \mathrm{C}$; IR (thin film, $\mathrm{cm}^{-1}$ ) 3448, 2933, 2871, 1780, $1741,1618,1446,1378,1320,1180,1135,1049,1024,1004,958,751 ;{ }^{1} \mathrm{H}$ NMR (600 $\left.\mathrm{MHz}, \mathrm{CDCl}_{3}\right) \delta 5.90(\mathrm{ddd}, J=10.2,4.8,1.2 \mathrm{~Hz}, 1 \mathrm{H}), 5.85(\mathrm{~m}, 1 \mathrm{H}), 5.72(\mathrm{~d}, J=10.2 \mathrm{~Hz}$, 1H), $5.07(\mathrm{~m}, 1 \mathrm{H}), 4.98(\mathrm{dd}, J=18.0,1.2 \mathrm{~Hz}, 1 \mathrm{H}), 4.80(\mathrm{dd}, J=18.0,1.8 \mathrm{~Hz}, 1 \mathrm{H}), 4.11$ (dd, $J=4.2,1.8 \mathrm{~Hz}, 1 \mathrm{H}), 3.97(\mathrm{~s}, 1 \mathrm{H}), 3.82(\mathrm{qd}, J=6.6,2.4 \mathrm{~Hz}, 1 \mathrm{H}), 3.74(\mathrm{br}, 1 \mathrm{H}), 2.77$ $(\mathrm{dd}, J=9.6,6.0 \mathrm{~Hz}, 1 \mathrm{H}), 2.25-2.05(\mathrm{~m}, 3 \mathrm{H}), 1.29(\mathrm{~d}, J=6.0 \mathrm{~Hz}, 3 \mathrm{H}), 1.80-1.05(\mathrm{~m}, 18 \mathrm{H})$, $0.92(\mathrm{~s}, 3 \mathrm{H}), 0.86(\mathrm{~s}, 3 \mathrm{H}) ;{ }^{13} \mathrm{C}$ NMR $\left(150 \mathrm{MHz}, \mathrm{CDCl}_{3}\right) \delta$ 174.6, 132.8, 127.5, 117.6, $93.2,85.5,73.6,73.4,69.7,67.9,64.9,50.90,49.5,41.8,40.0,36.4,35.7,35.1,33.1,30.7$, 30.3, 26.7 (2C), 26.5, 23.6, 21.3, 21.1, 17.9, 15.7; ESIHRMS Calcd for $\left[\mathrm{C}_{29} \mathrm{H}_{42} \mathrm{O}_{6} \mathrm{Na}^{+}\right]$: 509.2879, Found 509.2879. 


\section{Digitoxigen $\alpha$-L-rhamno-hexopyranoside (28):}

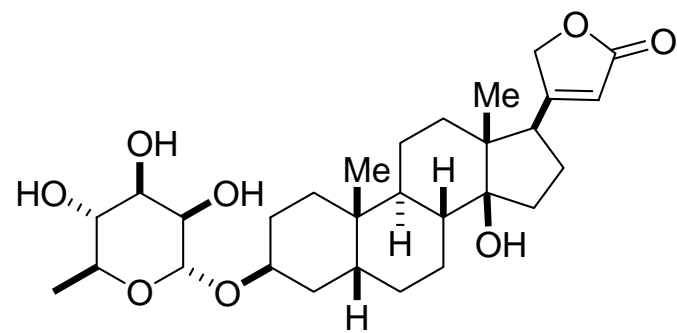

To a $t$-BuOH/acetone $(4 \mathrm{~mL})$ solution of alcohol $27(1.80 \mathrm{~g}, 3.82 \mathrm{mmol})$ at $0{ }^{\circ} \mathrm{C}$ was added a solution of $(50 \% \mathrm{w} / \mathrm{v})$ of $N$-methyl morpholine $N$-oxide/water $(2.4 \mathrm{~mL})$. Crystalline $\mathrm{OsO}_{4}(9.6 \mathrm{mg}, 1 \mathrm{~mol} \%)$ was added and the reaction was stirred for $4 \mathrm{~h}$. The reaction was quenched with adding EtOAc and satd. $\mathrm{NaHCO}_{3}$. The organic layer was separated and concentrated. The crude product was purified by a silica gel column using 90\% EtOAc/hexanes. Pure fractions were combined, concentrated, and crystallized from acetone/hexanes to afford triol $\mathbf{2 8}$ as a white solid $(2.07 \mathrm{~g}, 3.55 \mathrm{mmol}, 93 \%): \mathrm{R}_{\mathrm{f}}($ EtOAc) $=0.20 ; \mathrm{mp}: 224-225^{\circ} \mathrm{C} ;[\alpha]^{25}=-24.0(\mathrm{c}=0.7, \mathrm{MeOH}) ; \mathrm{IR}\left(\right.$ thin film, $\left.\mathrm{cm}^{-1}\right) 3453,2925$, $2856,1775,1736,1623,1449,1454,1378,1160,1076,1024,951,822 ;{ }^{1} \mathrm{H}$ NMR (600 $\left.\mathrm{MHz}, \mathrm{CDCl}_{3}\right) \delta 5.87(\mathrm{~m}, 1 \mathrm{H}), 4.98(\mathrm{dd}, J=18.0,1.2 \mathrm{~Hz}, 1 \mathrm{H}), 4.87(\mathrm{~m}, 1 \mathrm{H}), 4.80(\mathrm{dd}, J=$ 18.0, $1.2 \mathrm{~Hz}, 1 \mathrm{H}), 4.12(\mathrm{ddd}, J=3.0,3.0,3.0 \mathrm{~Hz}, 1 \mathrm{H}), 4.06(\mathrm{~m}, 1 \mathrm{H}), 3.71(\mathrm{dq}, J=9.0$, $6.0 \mathrm{~Hz}, 1 \mathrm{H}), 3.44(\mathrm{~m}, 1 \mathrm{H}), 2.77(\mathrm{~m}, 1 \mathrm{H}), 2.58(\mathrm{~s}, 1 \mathrm{H}), 2.36(\mathrm{~s}, 1 \mathrm{H}) 2.20-2.00(\mathrm{~m}, 4 \mathrm{H})$, $1.30(\mathrm{~d}, J=6.0 \mathrm{~Hz}, 3 \mathrm{H}), 1.90-1.10(\mathrm{~m}, 19 \mathrm{H}), 0.93(\mathrm{~s}, 3 \mathrm{H}), 0.87(\mathrm{~s}, 3 \mathrm{H}) ;{ }^{13} \mathrm{C} \mathrm{NMR}(150$ $\left.\mathrm{MHz}, \mathrm{CDCl}_{3}\right) \delta 174.48,174.45,117.8,97.5,85.6,73.8,73.4,71.9,71.6,67.8,50.9,49.6$, $41.9,40.1,38.3,36.4,35.7,35.2,33.2,30.3,29.5,26.6,26.5,26.4,23.7,21.4,21.2,17.5$, 15.8; ESIHRMS Calcd for $\left[\mathrm{C}_{29} \mathrm{H}_{44} \mathrm{O}_{8} \mathrm{Na}^{+}\right]$: 543.6446, Found 543.6446. 


\section{Digitoxigen 2,3-dideoxy-2,3-dihydro- $\alpha$-L-rhamno-hexopyranoside (29):}

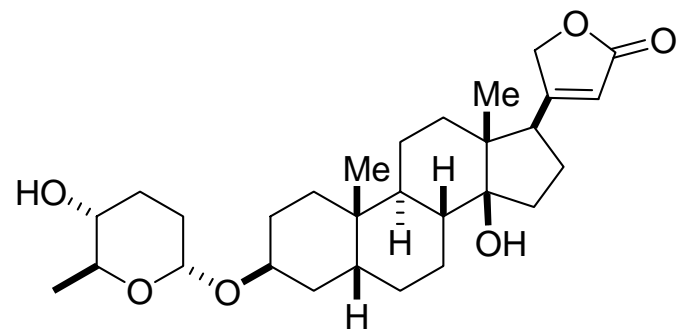

To a $\mathrm{CH}_{2} \mathrm{Cl}_{2}(0.35 \mathrm{~mL})$ solution of allylic alcohol $27(49 \mathrm{mg}, 0.1 \mathrm{mmol})$ was added $\mathrm{Et}_{3} \mathrm{~N}$ (50.6 mg, $0.5 \mathrm{mmol}$ ), o-nitrobenzenesulfonyl hydrazide (NBSH) (101.5 mg, $0.5 \mathrm{mmol})$. The reaction was stirred at room temperature for $6 \mathrm{~h}$. The reaction mixture was diluted with ether $(5 \mathrm{~mL})$ and was quenched with satd. aq $\mathrm{NaHCO}_{3}$, extracted $(3 \times 10 \mathrm{~mL})$ with $\mathrm{Et}_{2} \mathrm{O}$, dried $\left(\mathrm{Na}_{2} \mathrm{SO}_{4}\right)$, and concentrated under reduced pressure. The crude product was purified using silica gel flash chromatography eluting with $25 \%$ EtOAc/hexanes to give product 29 (47 mg, $0.096 \mathrm{mmol}, 96 \%)$ as a white solid: $\mathrm{R}_{\mathrm{f}}(50 \%$ EtOAc/hexanes $)=0.20$; mp: $180-181^{\circ} \mathrm{C} ;[\alpha]^{25}=-33.0(\mathrm{c}=0.4, \mathrm{MeOH}) ; \mathrm{IR}\left(\right.$ thin film, $\left.\mathrm{cm}^{-1}\right) 3441,2933,2246$, $1737,1619,1448,1379,1339,1258,1225,1115,1029,990,955,906,858,824 .{ }^{1} \mathrm{H}$ NMR $\left(600 \mathrm{MHz}, \mathrm{CDCl}_{3}\right) \delta 5.86(\mathrm{~m}, 1 \mathrm{H}), 4.98(\mathrm{dd}, J=18.0,1.2 \mathrm{~Hz}, 1 \mathrm{H}), 4.81(\mathrm{~m}, 1 \mathrm{H})$, $4.80(\mathrm{dd}, J=18.0,1.8 \mathrm{~Hz}, 1 \mathrm{H}), 4.11(\mathrm{dd}, J=4.2,1.8 \mathrm{~Hz}, 1 \mathrm{H}), 3.90$ (s, 1H), 3.63 (br, 1H), $3.25(\mathrm{~m}, 1 \mathrm{H}), 2.77(\mathrm{dd}, J=9.6,6.0 \mathrm{~Hz}, 1 \mathrm{H}), 2.25-2.05(\mathrm{~m}, 3 \mathrm{H}), 1.48(\mathrm{~s}, 1 \mathrm{H}), 1.20(\mathrm{~m}$, $6 \mathrm{H}), 1.80-1.05(\mathrm{~m}, 19 \mathrm{H}), 0.92(\mathrm{~s}, 3 \mathrm{H}), 0.86(\mathrm{~s}, 3 \mathrm{H}) ;{ }^{13} \mathrm{C} \mathrm{NMR}\left(150 \mathrm{MHz}, \mathrm{CDCl}_{3}\right) \delta 174.6$, $174.5,117.6,94.0,85.5,73.5,72.3,70.9,69.6,50.9,49.6,41.8,40.0,36.4,35.7,35.2$, 33.1, 30.5, 30.2, 29.8, 27.7, 26.9, 26.7 (2C), 23.7, 21.4, 21.1, 17.9, 15.7; ESIHRMS Calcd for $\left[\mathrm{C}_{29} \mathrm{H}_{44} \mathrm{O}_{6} \mathrm{Na}^{+}\right]:$511.6458, Found 511.6458. 
(2S, 6R)-2-Digitoxigenoxy-6-methyl-2H-pyran-3(6H)-one (32):

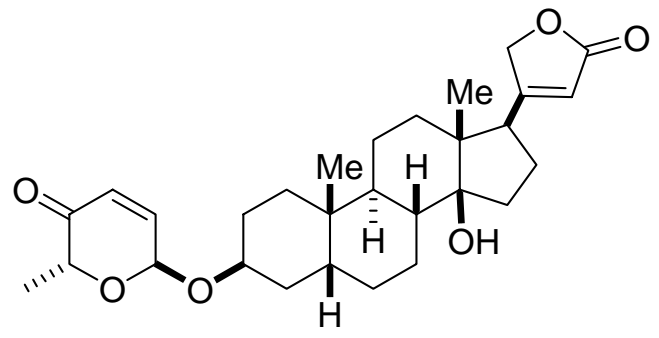

$\mathrm{A} \mathrm{CH}_{2} \mathrm{Cl}_{2} / \mathrm{THF}$ solution ( $3 \mathrm{~mL}, 4: 1 \mathrm{~V} / \mathrm{V}$ ) of Boc-pyranone $\mathbf{1 3 \beta}(200 \mathrm{mg}, 0.87 \mathrm{mmol}$ ) and digitoxigenin $(657 \mathrm{mg}, 1.75 \mathrm{mmol})$ was cooled to $0{ }^{\circ} \mathrm{C}$. $\mathrm{A} \mathrm{CH}_{2} \mathrm{Cl}_{2}(0.36 \mathrm{~mL})$ solution of $\mathrm{Pd}_{2}(\mathrm{DBA})_{3} \cdot \mathrm{CHCl}_{3}(26.2 \mathrm{mg}, 2.5 \mathrm{~mol} \%)$ and $\mathrm{PPh}_{3}(26.2 \mathrm{mg}, 10 \mathrm{~mol} \%)$ was added to the reaction mixture at $0{ }^{\circ} \mathrm{C}$. The reaction mixture was stirred at $0{ }^{\circ} \mathrm{C}$ for 8 hours and was quenched with $5 \mathrm{~mL}$ of satd. aq $\mathrm{NaHCO}_{3}$, extracted $(3 \times 10 \mathrm{~mL})$ with $\mathrm{Et}_{2} \mathrm{O}$, dried $\left(\mathrm{Na}_{2} \mathrm{SO}_{4}\right)$, and concentrated under reduced pressure. The crude product was purified using silica gel flash chromatography eluting with 40\% EtOAc/hexanes to give 32 (337 mg, $0.69 \mathrm{mmol}, 80 \%)$ as a white solid: $\mathrm{R}_{\mathrm{f}}(55 \%$ EtOAc/hexanes $)=0.45 ; \mathrm{mp}: 211-212{ }^{\circ} \mathrm{C}$; $[\alpha]^{25}=-27.0(\mathrm{c}=1.0, \mathrm{MeOH}) ; \mathrm{IR}\left(\right.$ thin film, $\left.\mathrm{cm}^{-1}\right)$ 3502, 2936, 2249, 1780, 1737, 1697, $1620,1447,1373,1338,1318,1235,1156,1103,1078,1023,958,909,824,754 ;{ }^{1} \mathrm{H}$ NMR $\left(600 \mathrm{MHz}, \mathrm{CDCl}_{3}\right) \delta 6.80(\mathrm{dd}, J=10.4,1.8 \mathrm{~Hz}, 1 \mathrm{H}), 6.12(\mathrm{dd}, J=10.2,1.2 \mathrm{~Hz}$, 1H), $5.87(\mathrm{~m}, 1 \mathrm{H}), 5.25(\mathrm{dd}, J=2.4,1.8 \mathrm{~Hz}, 1 \mathrm{H}), 4.99(\mathrm{dd}, J=18.0,1.8 \mathrm{~Hz}, 1 \mathrm{H}), 4.80$ (dd, $J=18.0,1.8 \mathrm{~Hz}, 1 \mathrm{H}), 4.08(\mathrm{~m}, 2 \mathrm{H}), 2.78(\mathrm{dd}, J=9.6,6.0 \mathrm{~Hz}, 1 \mathrm{H}), 2.20-2.09$ (m, $3 \mathrm{H}), 1.35(\mathrm{~d}, J=6.6 \mathrm{~Hz}, 3 \mathrm{H}), 1.92-1.16(\mathrm{~m}, 18 \mathrm{H}), 0.91(\mathrm{~s}, 3 \mathrm{H}), 0.85(\mathrm{~s}, 3 \mathrm{H}) ;{ }^{13} \mathrm{C} \mathrm{NMR}$ $\left(150 \mathrm{MHz}, \mathrm{CDCl}_{3}\right) \delta 197.0,174.4,174.3,147.7,128.2,117.8,93.9,85.6,75.2,73.4,73.3$, $50.9,49.6,41.9,40.0,36.3,35.8,35.2,33.2,32.0,30.0,26.9,26.4,24.4,23.7,21.3,21.2$, 17.0, 15.8; ESIHRMS Calcd for $\left[\mathrm{C}_{29} \mathrm{H}_{40} \mathrm{O}_{6} \mathrm{Na}^{+}\right]$: 507.2717, Found 507.2722. 


\section{(2S, 6R)-2-(Digitoxigenoxy)-2,5-dihydro-6-methyl-2H-pyran-5-ol (33):}

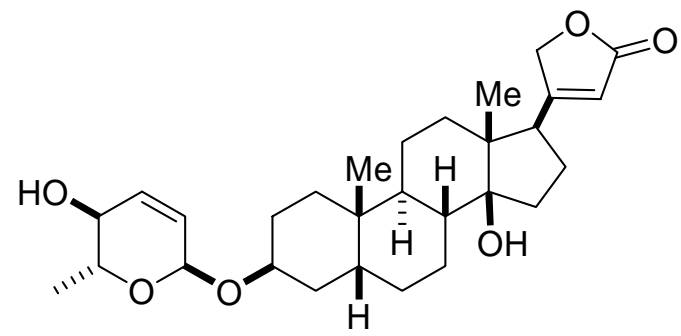

$\mathrm{A} \mathrm{CH}_{2} \mathrm{Cl}_{2}(1.12 \mathrm{~mL})$ solution of enone $33(270 \mathrm{mg}, 0.55 \mathrm{mmol})$ and $\mathrm{CeCl}_{3}$ in $\mathrm{MeOH}$ solution $(0.4 \mathrm{M}, 1.12 \mathrm{~mL})$ was cooled to $-78{ }^{\circ} \mathrm{C} . \mathrm{NaBH}_{4}(20.8 \mathrm{mg}, 0.55 \mathrm{mmol})$ was added and the reaction mixture was stirred at $-78{ }^{\circ} \mathrm{C}$ for $3 \mathrm{~h}$. The reaction mixture was diluted with ether $(10 \mathrm{~mL})$ and was quenched with $5 \mathrm{~mL}$ of satd. aq $\mathrm{NaHCO}_{3}$, extracted $(3 \times 10$ $\mathrm{mL}$ ) with $\mathrm{Et}_{2} \mathrm{O}$, dried $\left(\mathrm{Na}_{2} \mathrm{SO}_{4}\right)$, and concentrated under reduced pressure. The crude product was purified using silica gel flash chromatography eluting with 55\% EtOAc/hexanes to give allylic alcohol $33(238 \mathrm{mg}, 0.49 \mathrm{mmol}, 90 \%)$ as a white solid: $\mathrm{R}_{\mathrm{f}}$ $(60 \%$ EtOAc/hexanes $)=0.20 ; \mathrm{mp}: 198-199{ }^{\circ} \mathrm{C} ;$ IR (thin film, $\left.\mathrm{cm}^{-1}\right) 3327,2939,2871$, $1738,1741,1618,1448,1378,1320,1180,1135,1049,1024,958,750 ;{ }^{1} \mathrm{H}$ NMR (600 $\left.\mathrm{MHz}, \mathrm{CDCl}_{3}\right) \delta 5.90(\mathrm{ddd}, J=10.2,4.8,1.2 \mathrm{~Hz}, 1 \mathrm{H}), 5.86(\mathrm{~m}, 1 \mathrm{H}), 5.73(\mathrm{~d}, J=10.2 \mathrm{~Hz}$, 1H), $5.01(\mathrm{~m}, 1 \mathrm{H}), 4.97(\mathrm{dd}, J=18.0,1.2 \mathrm{~Hz}, 1 \mathrm{H}), 4.81(\mathrm{dd}, J=18.0,1.8 \mathrm{~Hz}, 1 \mathrm{H}), 4.00$ (dd, $J=4.2,1.8 \mathrm{~Hz}, 1 \mathrm{H}), 3.83(\mathrm{~s}, 1 \mathrm{H}), 3.73(\mathrm{qd}, J=6.6,2.4 \mathrm{~Hz}, 1 \mathrm{H}), 3.70(\mathrm{br}, 1 \mathrm{H}), 2.77$ $(\mathrm{dd}, J=9.6,6.0 \mathrm{~Hz}, 1 \mathrm{H}), 2.25-2.05(\mathrm{~m}, 3 \mathrm{H}), 1.29(\mathrm{~d}, J=6.0 \mathrm{~Hz}, 3 \mathrm{H}), 1.80-1.05(\mathrm{~m}, 18 \mathrm{H})$, 0.93 (s, 3H), 0.87 (s, 3H); ${ }^{13} \mathrm{C}$ NMR (150 MHz, $\left.\mathrm{CDCl}_{3}\right) \delta 174.5,174.4,144.3,127.0$, $117.6,91.6,85.5,73.76,73.4,70.3,50.9,49.6,41.8,40.0,36.7,35.6,35.2,33.1,32.0$, 30.1, 26.8 (2C), 26.5, 24.7, 23.7, 21.25, 21.16, 15.7, 15.2; ESIHRMS Calcd for $\left[\mathrm{C}_{29} \mathrm{H}_{42} \mathrm{O}_{6} \mathrm{Na}^{+}\right]$: 509.2879, Found 509.2879. 


\section{Digitoxigen 2,3-dideoxy-2,3-dihydro-a-D-rhamno-hexopyranoside (34):}

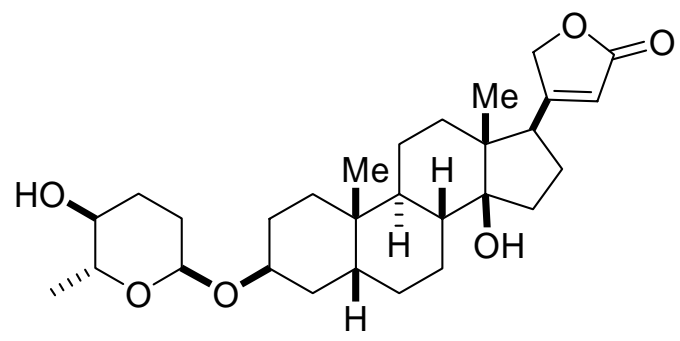

To a $\mathrm{CH}_{2} \mathrm{Cl}_{2}(0.35 \mathrm{~mL})$ solution of allylic alcohol $\mathbf{3 3}(50 \mathrm{mg}, 0.1 \mathrm{mmol})$ was added $\mathrm{Et}_{3} \mathrm{~N}$ (50.6 mg, $0.5 \mathrm{mmol}$ ), o-nitrobenzenesulfonyl hydrazide (NBSH) (101.5 mg, $0.5 \mathrm{mmol})$. The reaction was stirred at room temperature for $6 \mathrm{~h}$. The reaction mixture was diluted with ether $(5 \mathrm{~mL})$ and was quenched with satd. aq $\mathrm{NaHCO}_{3}$, extracted $(3 \times 10 \mathrm{~mL})$ with $\mathrm{Et}_{2} \mathrm{O}$, dried $\left(\mathrm{Na}_{2} \mathrm{SO}_{4}\right)$, and concentrated under reduced pressure. The crude product was purified using silica gel flash chromatography eluting with $25 \%$ EtOAc/hexanes to give product 29 (46.5 $\mathrm{mg}, 0.095 \mathrm{mmol}, 95 \%)$ as a white solid: $\mathrm{R}_{\mathrm{f}}(50 \%$ EtOAc/hexanes $)=$ 0.20; mp: $180-181^{\circ} \mathrm{C} ;[\alpha]^{25}=-33.0(\mathrm{c}=0.4, \mathrm{MeOH}) ;$ IR (thin film, $\left.\mathrm{cm}^{-1}\right) 3434,2932$, $2242,1780,1736,1619,1447,1379,1338,1225,1149,1115,1029,989,956,908,857$, 824; ${ }^{1} \mathrm{H}$ NMR $\left(600 \mathrm{MHz}, \mathrm{CDCl}_{3}\right) \delta 5.86(\mathrm{~m}, 1 \mathrm{H}), 4.98(\mathrm{dd}, J=18.0,1.2 \mathrm{~Hz}, 1 \mathrm{H}), 4.81$ (m, 1H), $4.80(\mathrm{dd}, J=18.0,1.8 \mathrm{~Hz}, 1 \mathrm{H}), 4.00(\mathrm{dd}, J=4.2,1.8 \mathrm{~Hz}, 1 \mathrm{H}), 3.92(\mathrm{~s}, 1 \mathrm{H}), 3.63$ (br, 1H), $3.25(\mathrm{~m}, 1 \mathrm{H}), 2.77(\mathrm{dd}, J=9.6,6.0 \mathrm{~Hz}, 1 \mathrm{H}), 2.25-2.05(\mathrm{~m}, 3 \mathrm{H}), 1.48(\mathrm{~s}, 1 \mathrm{H})$, $1.20(\mathrm{~m}, 6 \mathrm{H}), 1.80-1.05(\mathrm{~m}, 19 \mathrm{H}), 0.93(\mathrm{~s}, 3 \mathrm{H}), 0.87(\mathrm{~s}, 3 \mathrm{H}) ;{ }^{13} \mathrm{C} \mathrm{NMR}\left(150 \mathrm{MHz}, \mathrm{CDCl}_{3}\right)$ $\delta 174.6,174.5,117.6,93.9,85.5,73.5,72.3,70.7,69.6,50.9,49.6,41.9,40.1,36.7,35.7$, 35.2, 33.1, 32.0, 30.2, 30.1, 27.7, 26.9, 26.7 (2C), 23.7, 21.4, 21.1, 17.9, 15.8; ESIHRMS Calcd for $\left[\mathrm{C}_{29} \mathrm{H}_{44} \mathrm{O}_{6} \mathrm{Na}^{+}\right]$: 511.6458, Found 511.6458. 


\section{L- $\beta$-glycoside desacetoxy-oleandrin (36):}

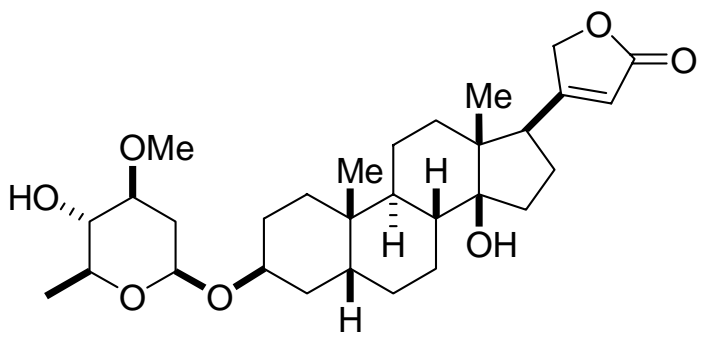

To a mixture of diol 25 (25 mg, $0.05 \mathrm{mmol})$ and silver (I) oxide (0.23 g, $1 \mathrm{mmol})$, was added $0.25 \mathrm{~mL} \mathrm{CH}_{3} \mathrm{I}$. The reaction suspension was stirred at $\mathrm{rt}$ for 3 days. The reaction mixture was then passed through a celite pad with $20 \mathrm{~mL} \mathrm{Et}_{2} \mathrm{O}$, concentrated under reduced pressure and purified using silica flash chromatography eluting with $50 \%$ EtOAc/hexanes to give product $36(6.49 \mathrm{mg}, 0.012 \mathrm{mmol}, 25 \%)$ as a white solid: $\mathrm{R}_{\mathrm{f}}(50 \%$ EtOAc/hexanes $)=0.20 ; \mathrm{mp}: 185-186^{\circ} \mathrm{C} ;[\alpha]^{25}{ }_{\mathrm{D}}=+43.0(\mathrm{c}=0.45, \mathrm{MeOH}) ; \mathrm{IR}$ (thin film, $\left.\mathrm{cm}^{-1}\right) 3453,2932,2856,2173,1969,1775,1742,1623,1449,1454,1378,1160,1067$, 1024, 951, 822, 731; ${ }^{1} \mathrm{H}$ NMR (600 MHz, $\left.\mathrm{CDCl}_{3}\right) \delta 5.86(\mathrm{~m}, 1 \mathrm{H}), 4.98(\mathrm{dd}, J=18.0,1.8$ Hz, 1H), $4.80(\mathrm{dd}, J=18.0,1.8 \mathrm{~Hz}, 1 \mathrm{H}), 4.53(\mathrm{dd}, J=9.6,1.8 \mathrm{~Hz}, 1 \mathrm{H}), 4.02(\mathrm{~m}, 1 \mathrm{H})$, $3.58(\mathrm{dq}, J=9.0,6.0 \mathrm{~Hz}, 1 \mathrm{H}), 3.40(\mathrm{~s}, 3 \mathrm{H}), 3.24(\mathrm{~m}, 1 \mathrm{H}), 3.09(\mathrm{~m}, 1 \mathrm{H}), 2.77(\mathrm{~m}, 1 \mathrm{H})$, $2.33(\mathrm{~s}, 1 \mathrm{H}), 2.20-2.00(\mathrm{~m}, 4 \mathrm{H}), 1.26(\mathrm{~d}, J=6.0 \mathrm{~Hz}, 3 \mathrm{H}), 1.90-1.10(\mathrm{~m}, 21 \mathrm{H}), 0.92(\mathrm{~s}$, $3 \mathrm{H}), 0.86(\mathrm{~s}, 3 \mathrm{H}) ;{ }^{13} \mathrm{C} \mathrm{NMR}\left(150 \mathrm{MHz}, \mathrm{CDCl}_{3}\right) \delta 174.5,174.4,117.7,97.5,85.6,81.0$, $73.4,72.9,71.8,71.6,56.2,50.9,49.6,41.8,40.0,39.3,36.2,35.7,35.2,33.2,32.0,29.9$, 26.9, 26.4, 24.4, 23.6, 21.2, 21.1, 17.8, 15.8; ESIHRMS Calcd for $\left[\mathrm{C}_{30} \mathrm{H}_{46} \mathrm{O}_{7} \mathrm{Na}^{+}\right]$: 541.3141, Found 541.3141. 


\section{References:}

1. (a) Toshima, K.; Tatsuta, K. Chem. Rev. 1993, 93, 1503-1531. (b) Danishefsky, S.

J.; Bilodeau, M. T. Angew. Chem., Int. Ed. Engl. 1996, 35, 1380-1419. (c) Kirschning, A.; Bechthold, A. F.-W.; Rohr, J. Top. Curr. Chem. 1997, 188, 1-84. (d) Marzabadi, C. H.; Frank, R. W. Tetrahedron 2000, 56, 8385-8417. (e) Kirschning, A.; Jesberger, M.; Schoning, K. U. Synthesis 2001, 507-540. (f) Nicolaou, K. C.; Mitchell, H. J. Angew. Chem., Int. Ed. 2001, 40, 1576-1624. (g) Sears, P.; Wong, C.-H. Science 2001, 291, 2344-2350. (h) He, X.; Liu, H.-W. Annu. Rev. Biochem. 2002, 71, 701-754.

2. (a) Haux; J. Med. Hypotheses 1999, 53, 543-548. (b) Kometiani, P.; Liu, L.; Askari, A. Mol. Pharmacol. 2005, 67, 929-936. (c) Lopez-Lazaro, M.; Pastor, N.; Azrak, S. S.; Ayuso, M. J.; Austin, C. A.; Cortes, F. J. Nat. Prod. 2005, 68, 1642-1645. (d) Winnicka, K. Bielawski, K.; Bielawska, A. Acta Pol. Pharm. 2006, 63, 109-115.

3. For the synthesis of digitoxigenin, see: (a) Danieli, N.; Mazur, Y.; Sondheimer, F. J. Am. Chem. Soc. 1962, 84, 875-876. (b) Donovan, Stephen, F.; Avery, Mitchell, A.; McMurry, John, E. Tetrahedron Lett. 1979, 35, 3287-3290. (c) Tsai, T. Y.R.; Minta, A.; Wiesner, K. Heterocycles 1979, 12, 1397-1402. (d) Marini-Bettolo, R.; Flecker, P.; Tsai, T. Y.R.; Wiesner, K. Can. J. Chem. 1981, 59, 1403-1410. (e) Kabat, M. M. J. Org. Chem. 1995, 60, 1823-1827. (f) Stork, G.; West, F.; Lee, H. Y.; Isaacs, R. C. A.; Manabe, S. J. Am. Chem. Soc. 1996, 118, 10660-10661. (g) Almirante, N.; Cerri, A. J. Org. Chem. 1997, 62, 3402-3404. (h) Bocknack, B. M.; Wang, L.-C.; Krische, M. J. Proc. Natl. Acad. Sci. U.S.A. 2004, 101, 5421-5424. 
4. The attempts at the selective hydrolysis of digitoxin to form digoxose has been futile, only the monosaccharide digitoxose was isolated. Surprisingly, digoxose can be isolated from the dried twigs of Orthenthera viminea, see: Tiwari, K. N.; Khare, N. K.; Khare, A.; Khare, M. P. Carbohydr. Res. 1984, 129, 179-187.

5. (a) Wiesner, K.; Tsai, T. Y. R.; Jin, H. Helv. Chim. Acta 1985, 68, 300-314. (b) Wiesner, K.; Tsai, T. Y. R. Pure Appl. Chem. 1986, 58, 799-810. (c) McDonald, F. E.; Reddy, K. S.; Diaz, Y. J. Am. Chem. Soc. 2000, 122, 4304-4309. (d) McDonald, F. E.; Reddy, K. S. Angew. Chem., Int. Ed. 2001, 40, 3653-3655

6. There have been 8 syntheses of digitoxigenin. See ref 3 .

7. Zhou, M.; O’Doherty, G.; J. Org. Chem. 2007, 72 (7), 2485 -2493.

8. Samples were sent and tested by NCI.

9. (a) Fujii, A.; Hashiguchi, S.; Uematsu, N.; Ikariya, T.; Noyori, R. J. Am. Chem. Soc. 1996, 118, 2521-2522. (b) Li, M.; Scott, J. G.; O'Doherty, G. A. Tetrahedron Lett. 2004, 45, 1005-1009. (c) Li, M.; O'Doherty, G. A. Tetrahedron Lett. 2004, 45, 6407-6411.

10. (a) Babu, R. S.; O'Doherty, G. A. J. Carbohydr. Chem. 2005, 24, 169-177. (b) Guo, H.; O'Doherty, G. A. Org. Lett. 2005, 7, 3921-3924.

11. Mitsunobu, O.; Wada, M.; Sano, T. J. Am. Chem. Soc. 1972, 94, 679.

12. Samples were sent and tested by Dr. Joseph Langenhan, Department of Chemistry, Seattle University.

13. Guo, H.; O'Doherty, G. A. Angew. Chem., Int. Ed. 2007, 46, 5206-5208.

14. Li, M.; Scott, J.; O’Doherty, G. A. Tetrahedron Lett. 2004, 45, 1005-1009. 
Appendix

List of ${ }^{1} \mathrm{H}$ and ${ }^{13} \mathrm{C}$ NMR Spectra 


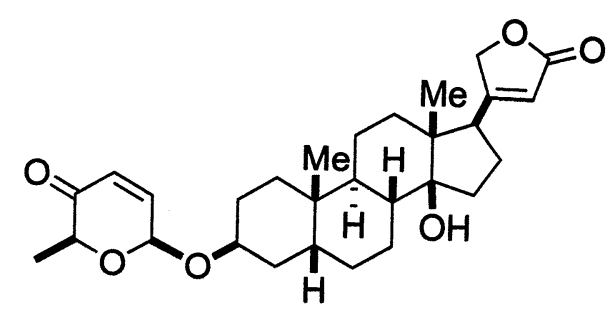

${ }^{1} \mathrm{H}$ NMR (600 MHz, $\mathrm{CDCl}_{3}$ )

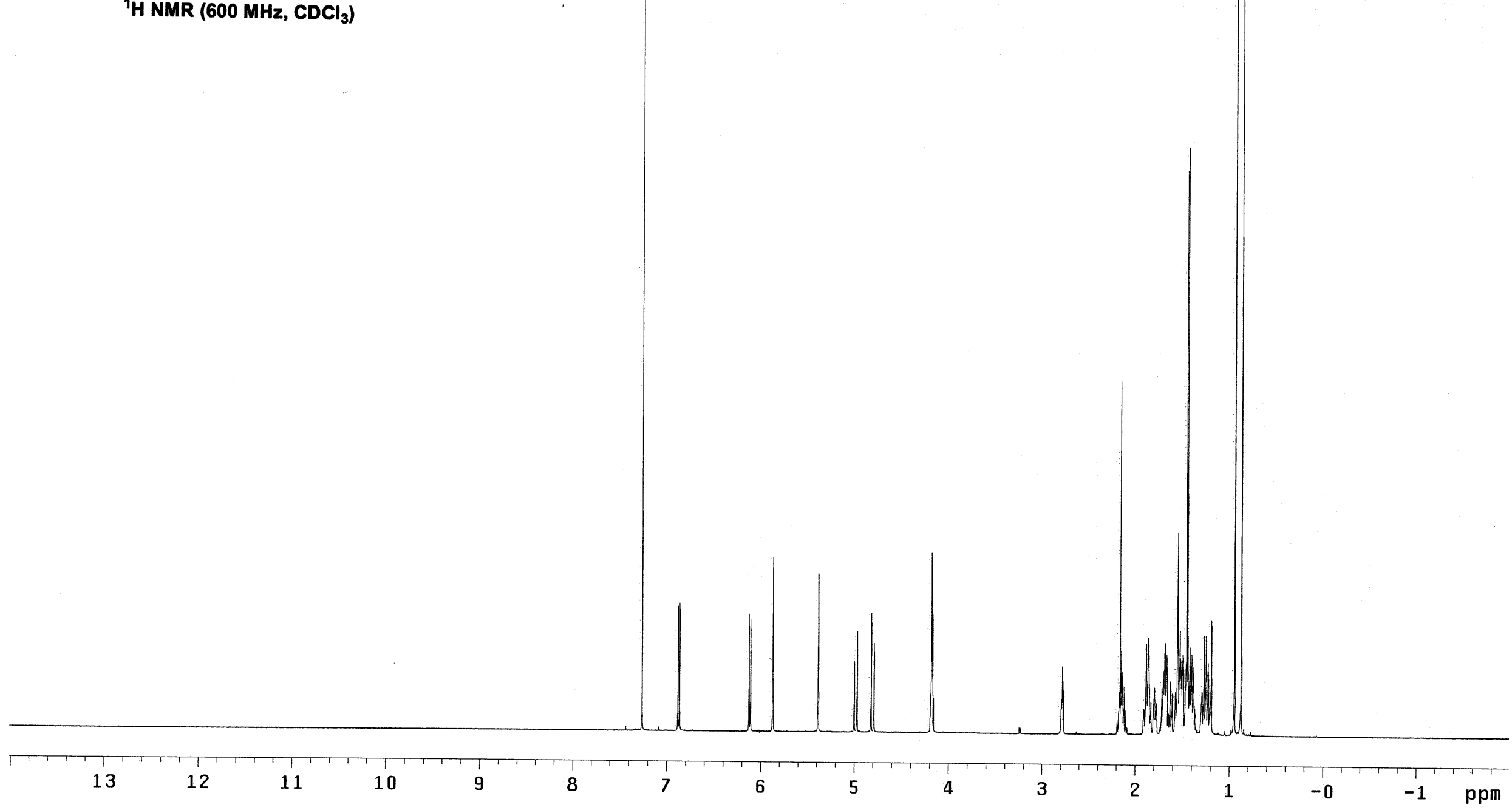




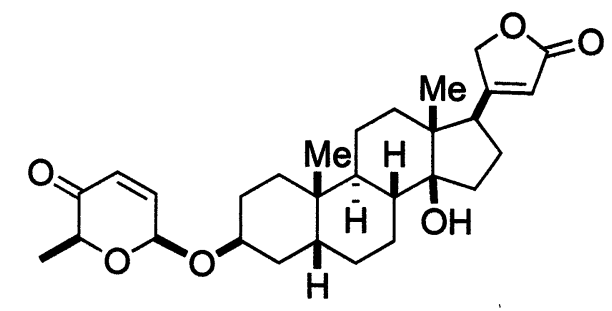

${ }^{13} \mathrm{C}$ NMR (150 $\left.\mathrm{MHz} \mathrm{CDCl}_{3}\right)$ 


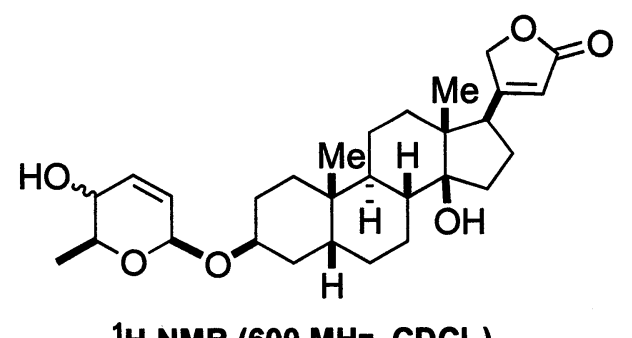

${ }^{1} \mathrm{H}$ NMR (600 MHz, $\mathrm{CDCl}_{3}$ )

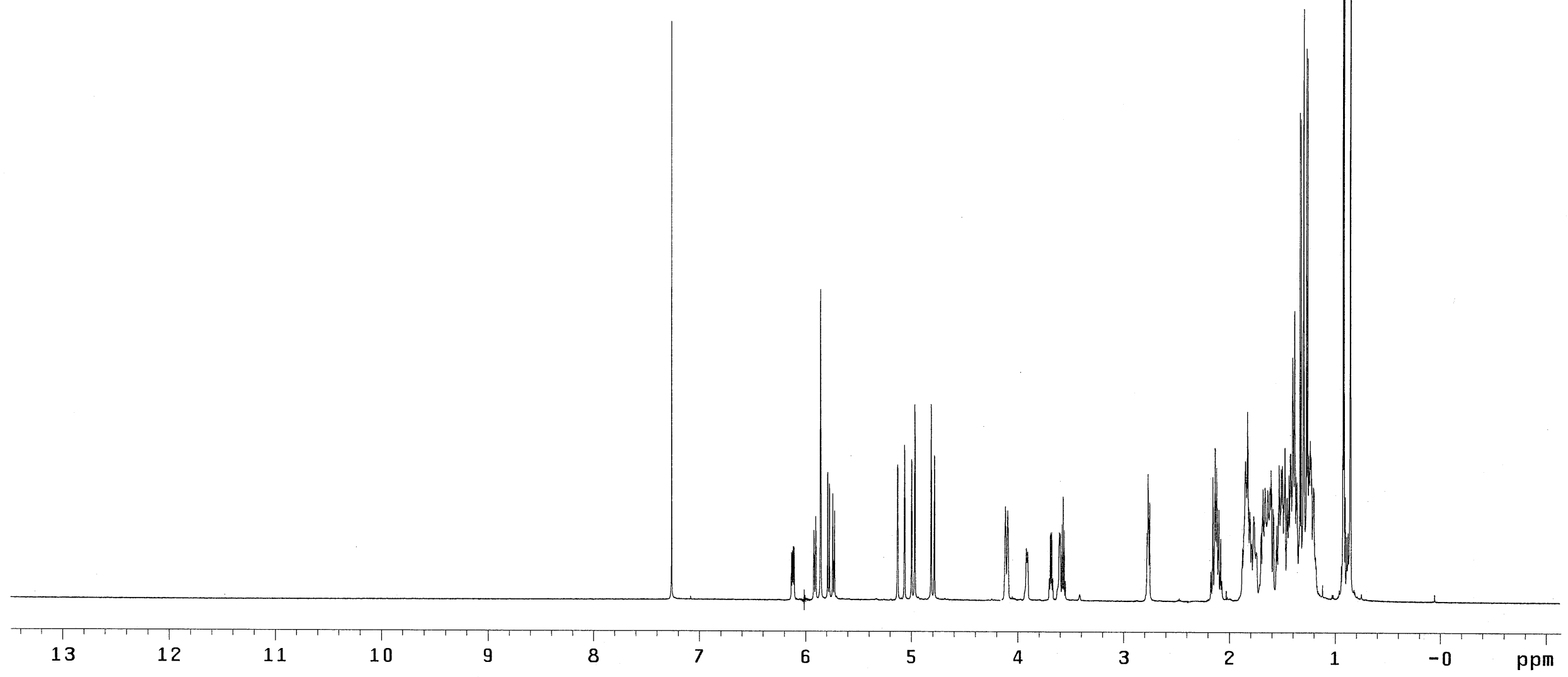




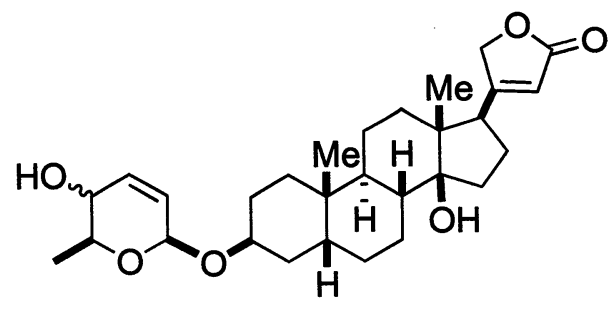

${ }^{13} \mathrm{C}$ NMR (150 MHz, $\left.\mathrm{CDCl}_{3}\right)$<smiles>CCC</smiles> 


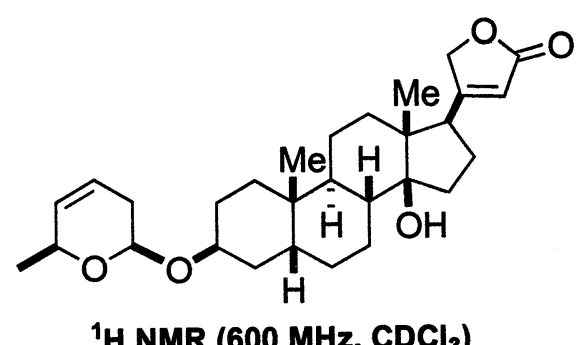

${ }^{1} \mathrm{H} \mathrm{NMR}\left(600 \mathrm{MHz}, \mathrm{CDCl}_{3}\right)$

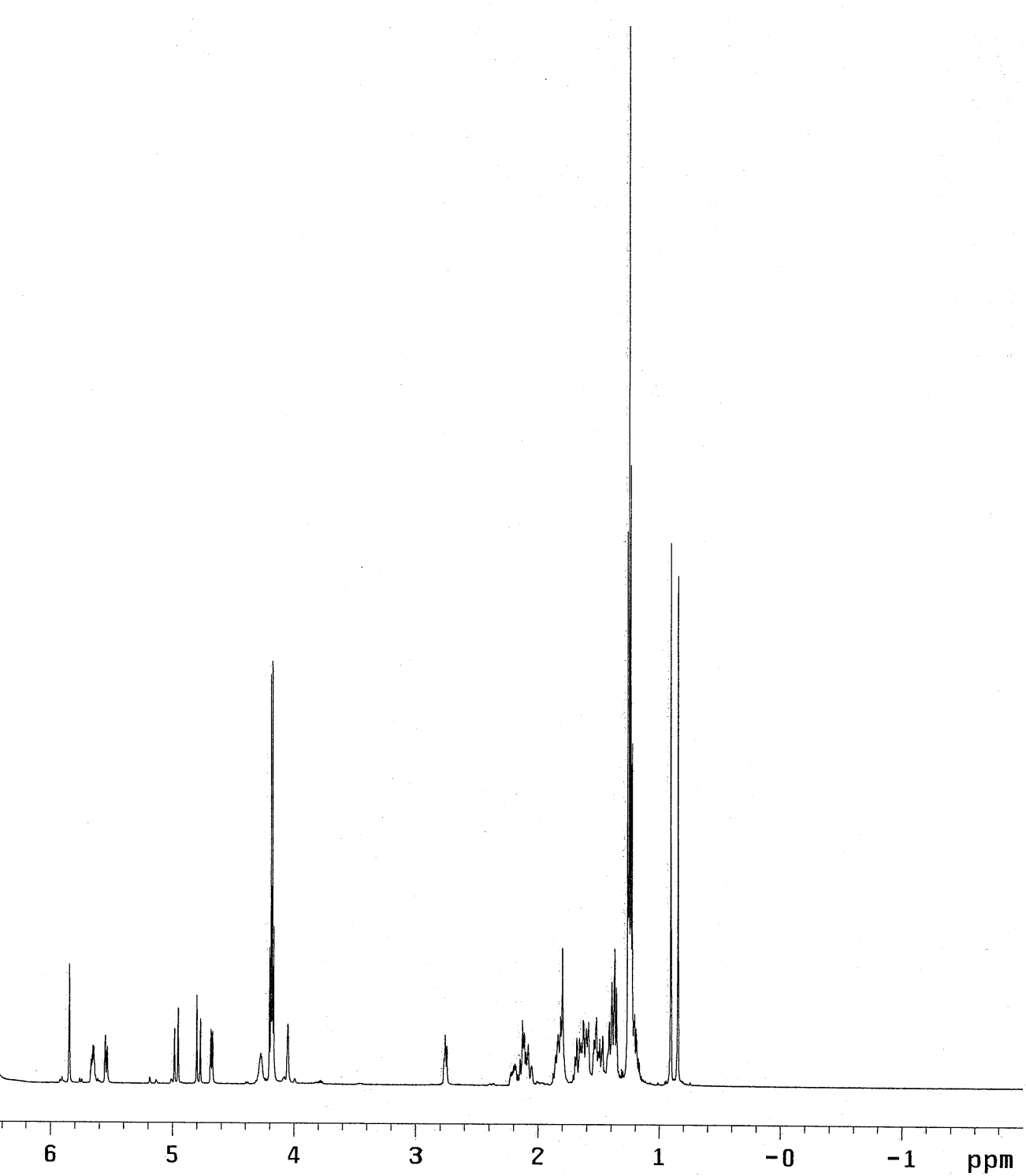




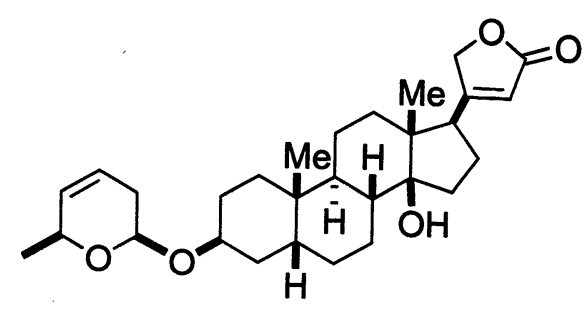

$\left.{ }^{13} \mathrm{C} \mathrm{NMR} \mathrm{(150} \mathrm{MHz,} \mathrm{CDCl}_{3}\right)$

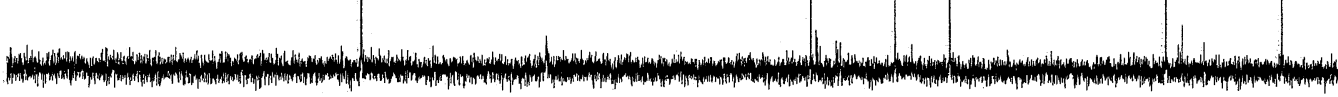

$\begin{array}{llccccccccc}200 & 180 & 160 & 140 & 120 & 100 & 80 & 60 & 40 & 20 & 0 \\ & & 45 & & \end{array}$




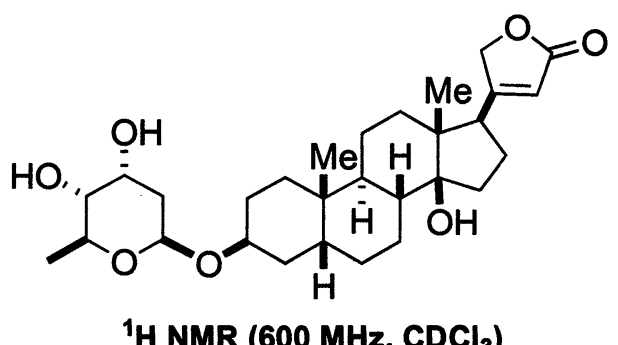

${ }^{1} \mathrm{H}$ NMR (600 MHz, $\mathrm{CDCl}_{3}$ )

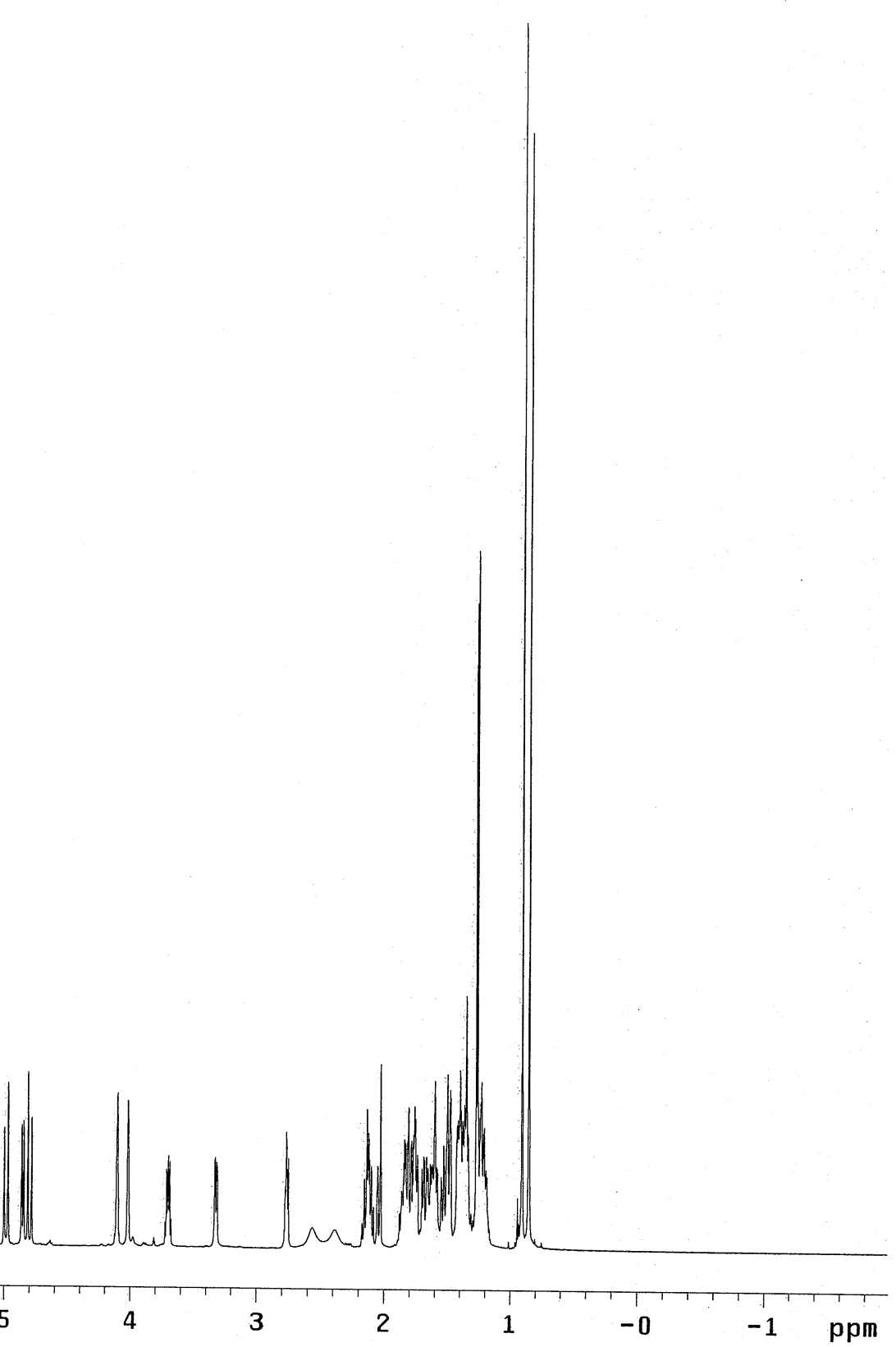




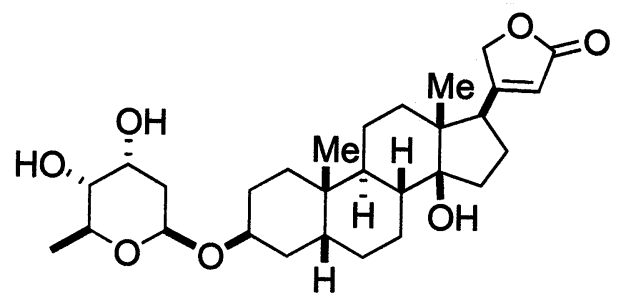

${ }^{13} \mathrm{C} \mathrm{NMR} \mathrm{(150} \mathrm{MHz,} \mathrm{CDCl}_{3}$ )
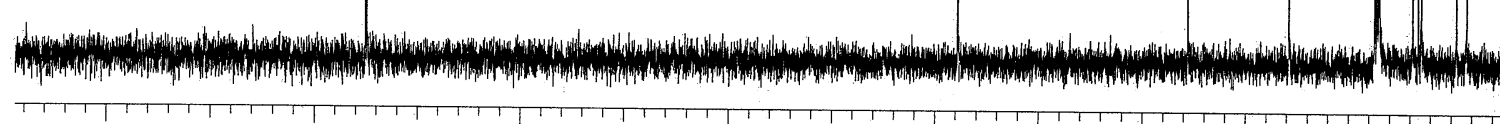


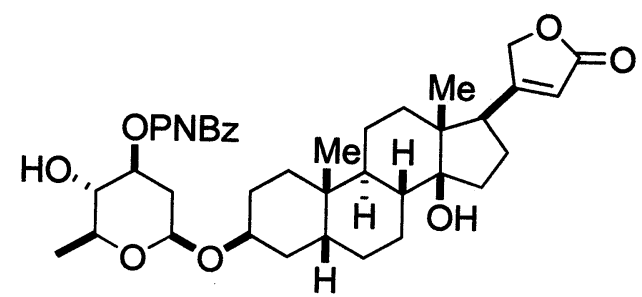

${ }^{1} \mathrm{H} \mathrm{NMR}\left(600 \mathrm{MHz}, \mathrm{CDCl}_{3}\right)$

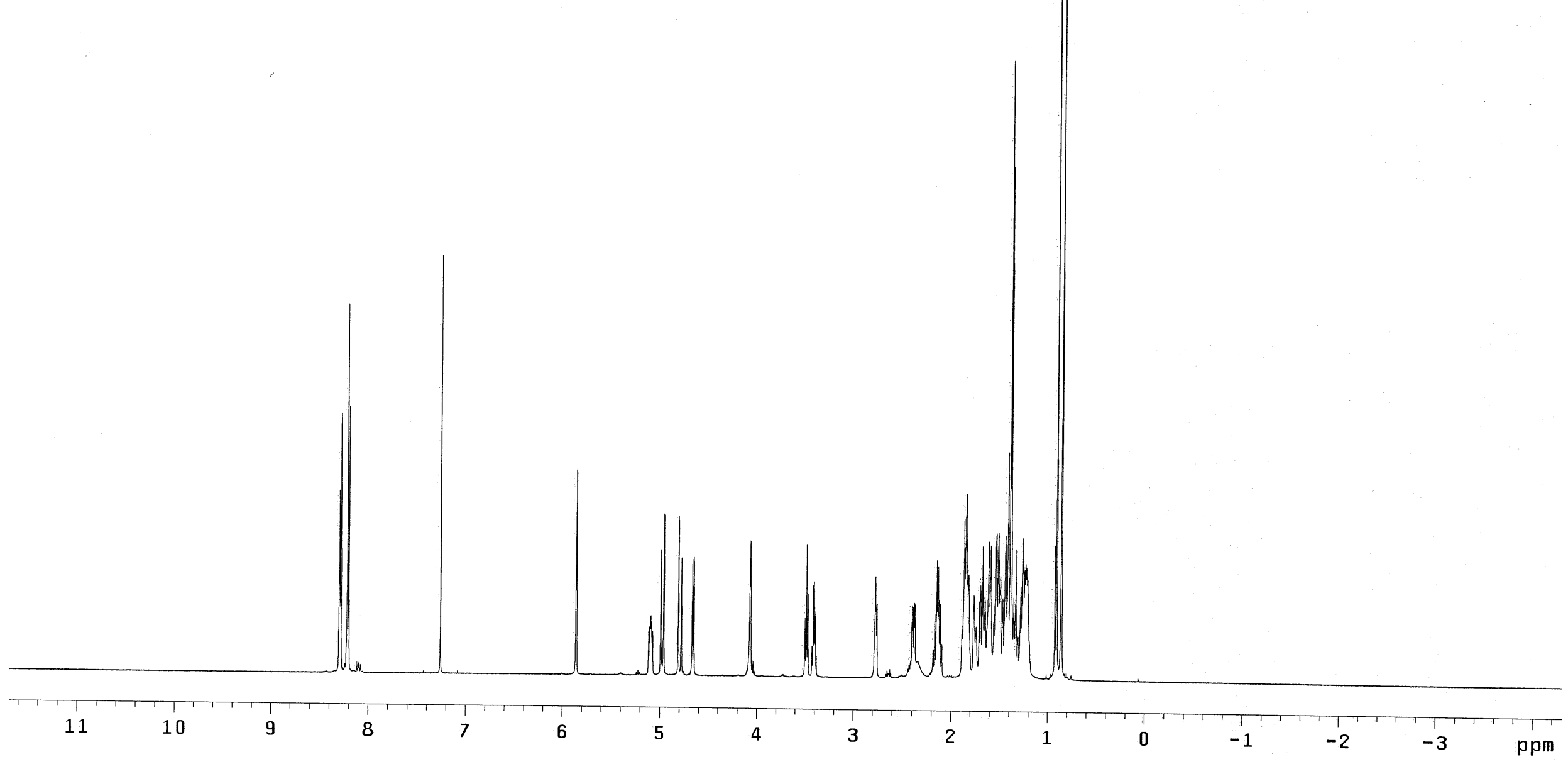




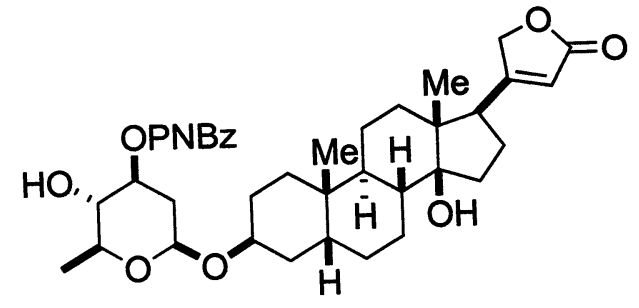

${ }^{13} \mathrm{C}$ NMR $\left(150 \mathrm{MHz}, \mathrm{CDCl}_{3}\right)$

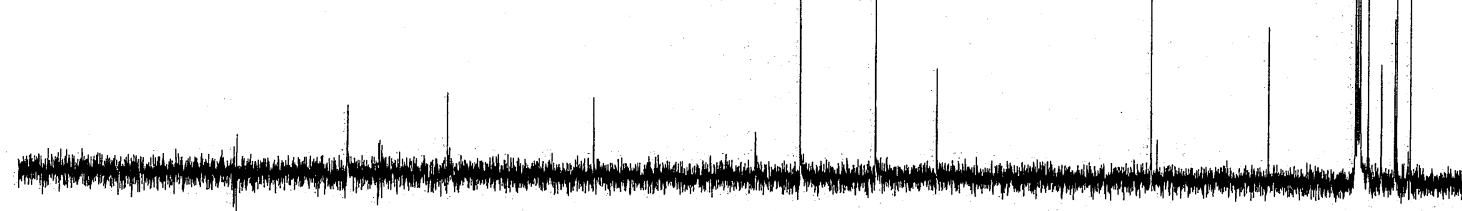

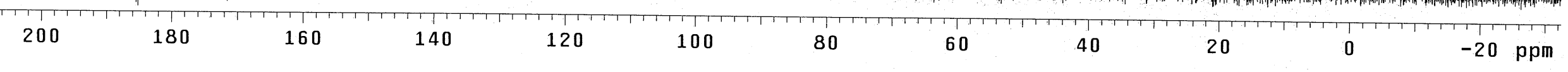




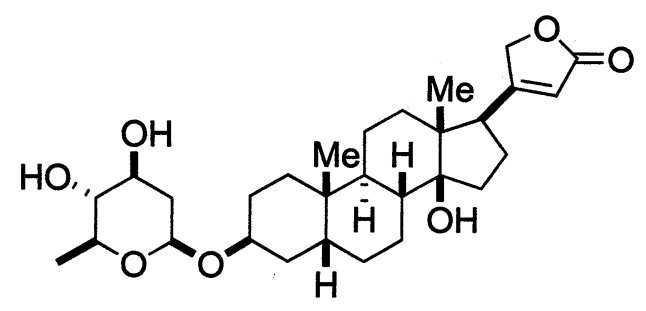

${ }^{1} \mathrm{H}$ NMR (600 MHz, $\left.\mathrm{CDCl}_{3}\right)$

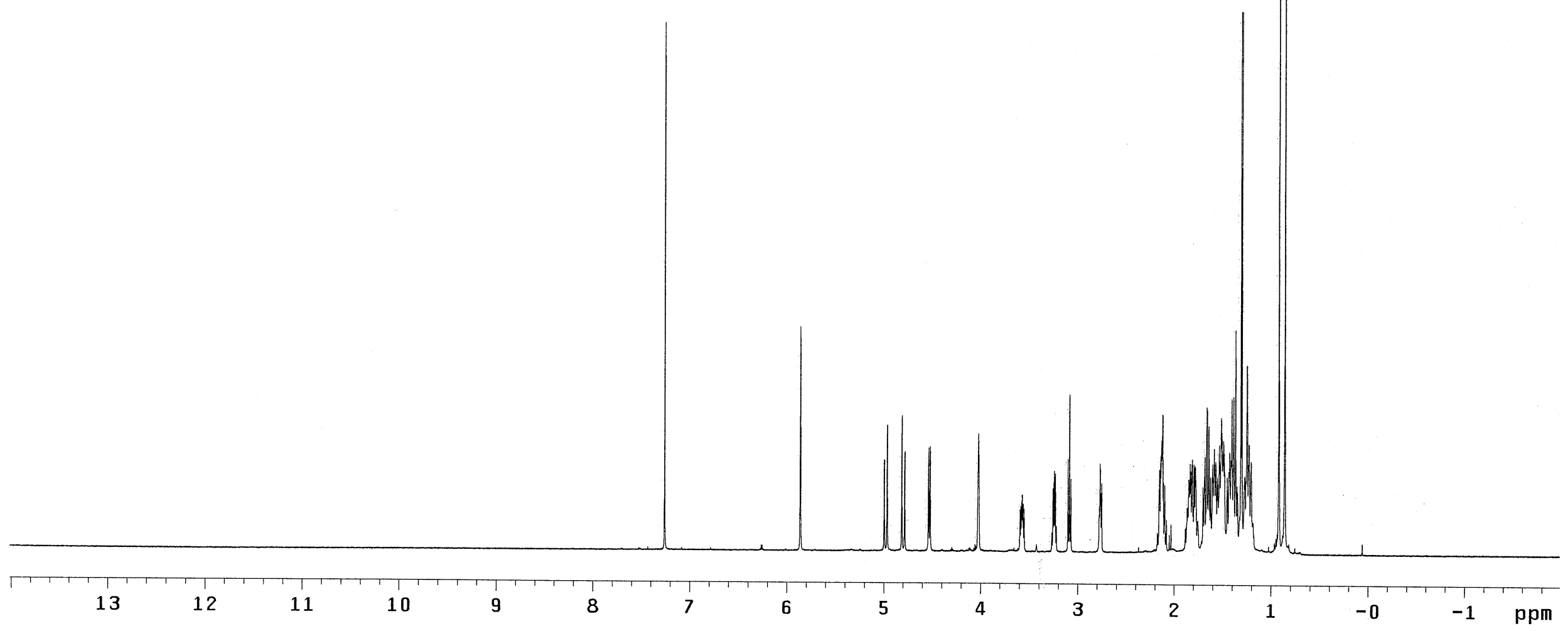




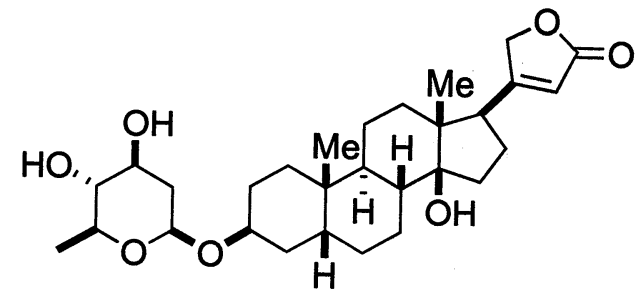

${ }^{13} \mathrm{C}$ NMR (150 $\left.\mathrm{MHz}, \mathrm{CDCl}_{3}\right)$

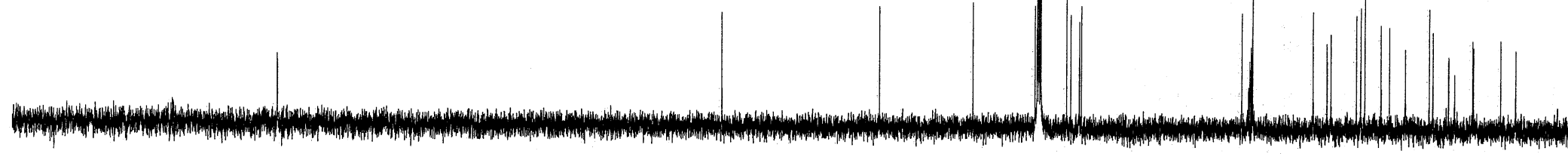

$\begin{array}{ccccc}200 & 180 & 160 & 140 & 120\end{array}$




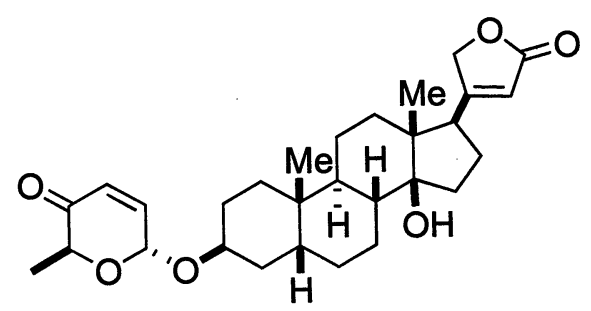

${ }^{1} \mathrm{H}$ NMR $\left(600 \mathrm{MHz}, \mathrm{CDCl}_{3}\right)$

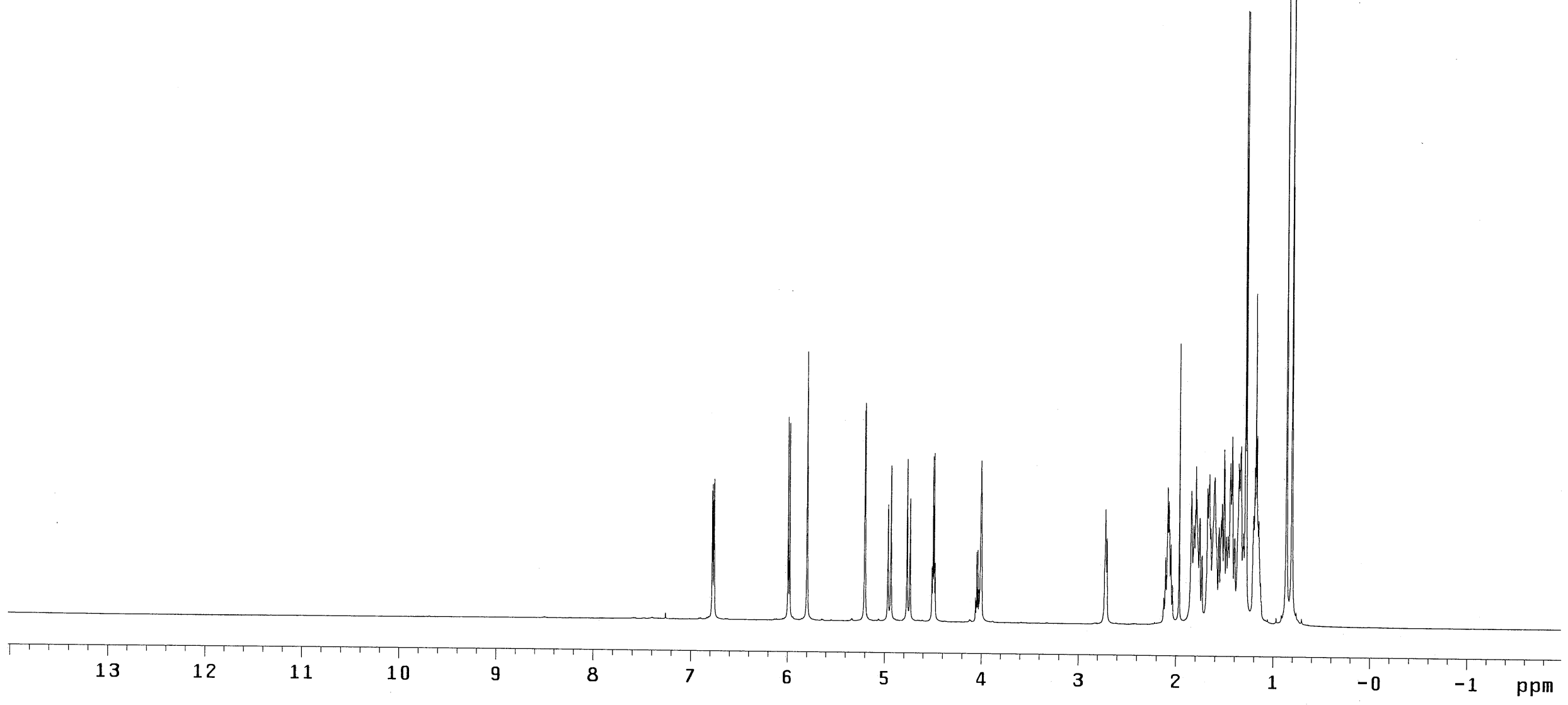




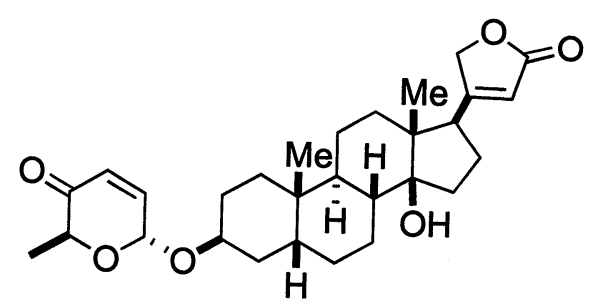

${ }^{13} \mathrm{C}$ NMR (150 MHz, $\left.\mathrm{CDCl}_{3}\right)$ 


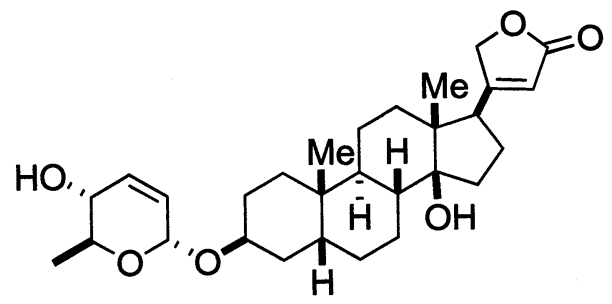

${ }^{1} \mathrm{H}$ NMR (600 MHz, $\mathrm{CDCl}_{3}$ )

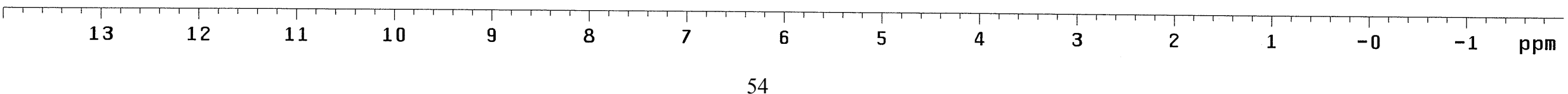




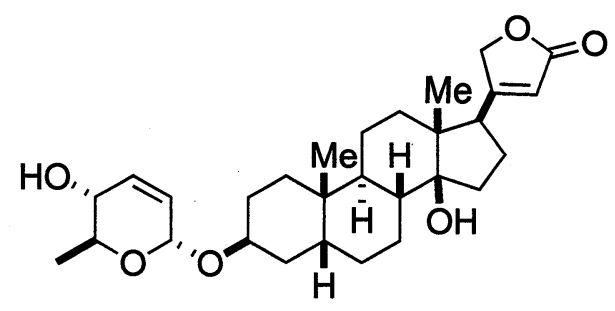

${ }^{13} \mathrm{C}$ NMR (150 MHz, $\mathrm{CDCl}_{3}$ )

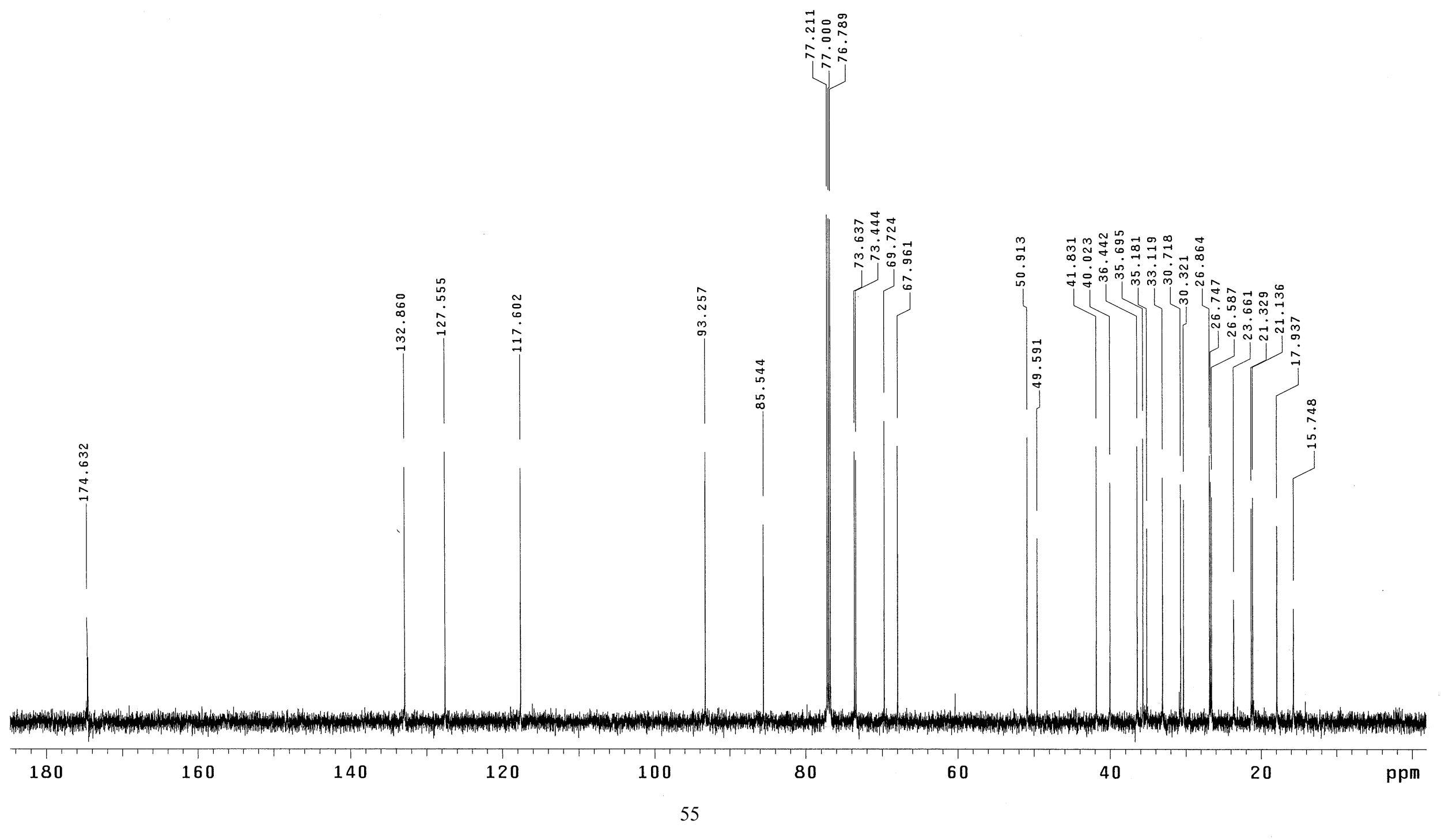




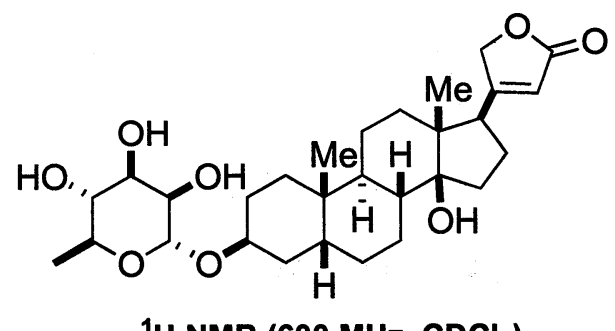

${ }^{1} \mathrm{H}$ NMR (600 MHz, $\mathrm{CDCl}_{3}$ ) 


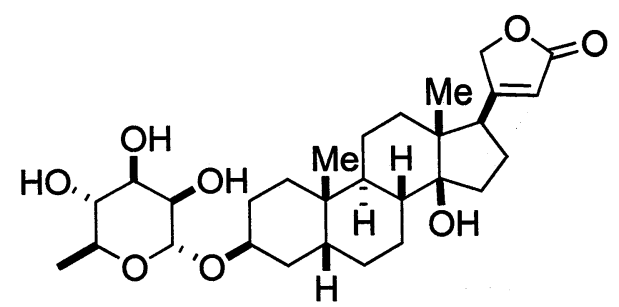

${ }^{13} \mathrm{C}$ NMR (150 MHz, $\mathrm{CDCl}_{3}$ ) 


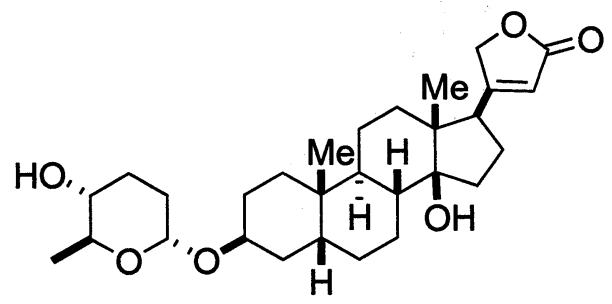

${ }^{1} \mathrm{H}$ NMR (600 MHz, $\mathrm{CDCl}_{3}$ )

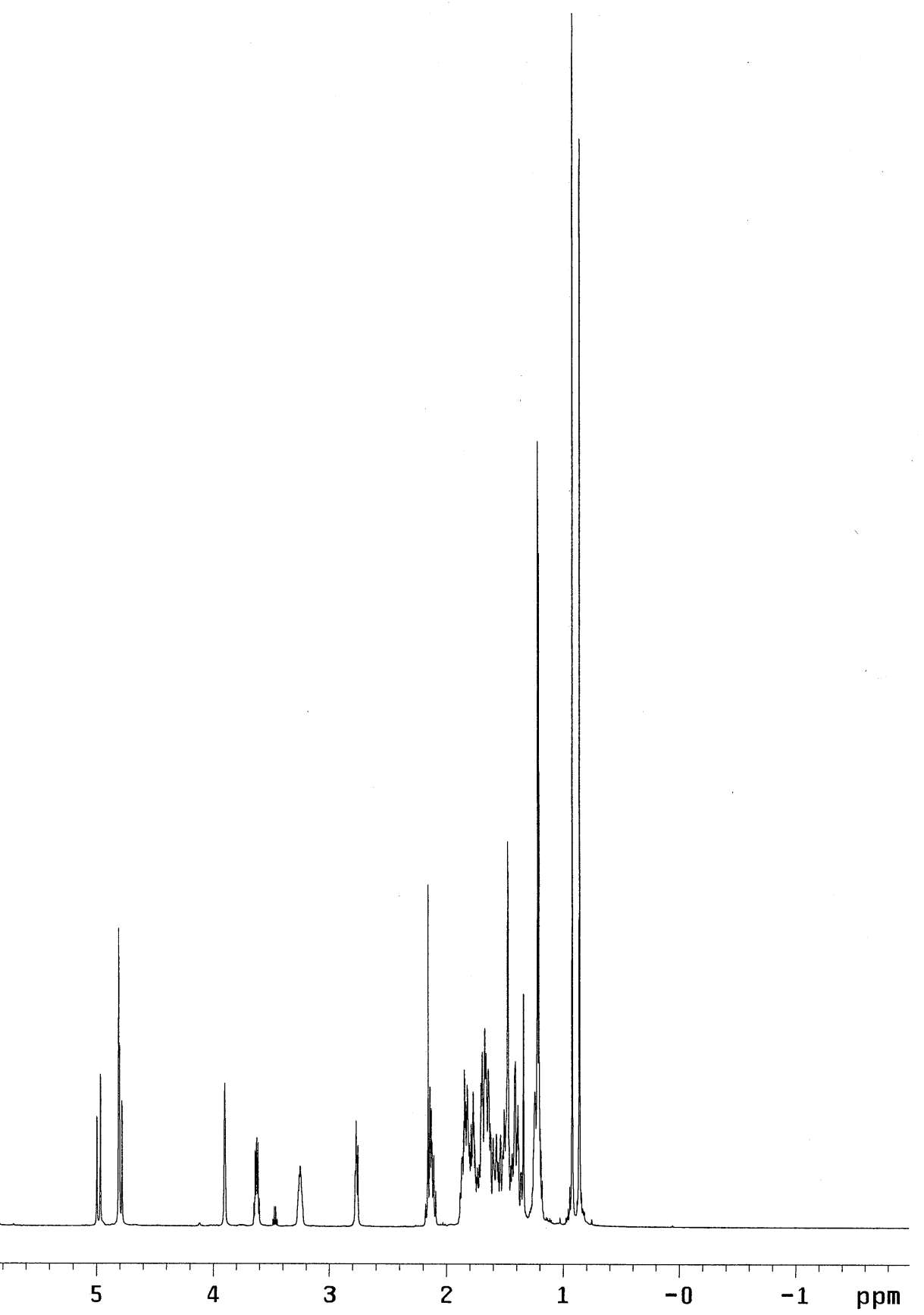




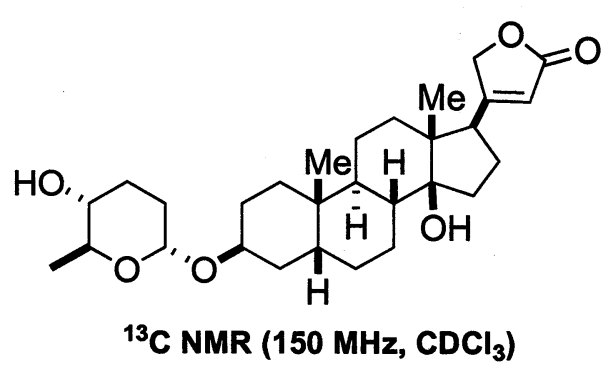

${ }^{13} \mathrm{C}$ NMR (150 MHz, $\left.\mathrm{CDCl}_{3}\right)$ 


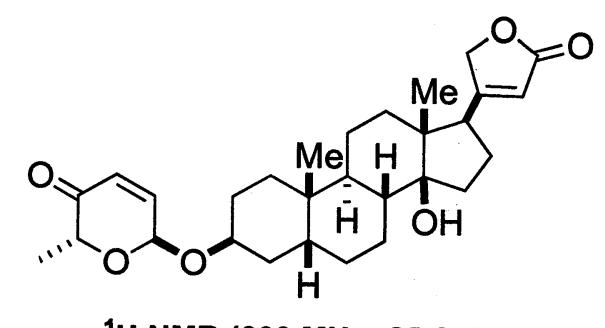

${ }^{1} \mathrm{H}$ NMR (600 MHz, $\mathrm{CDCl}_{3}$ )

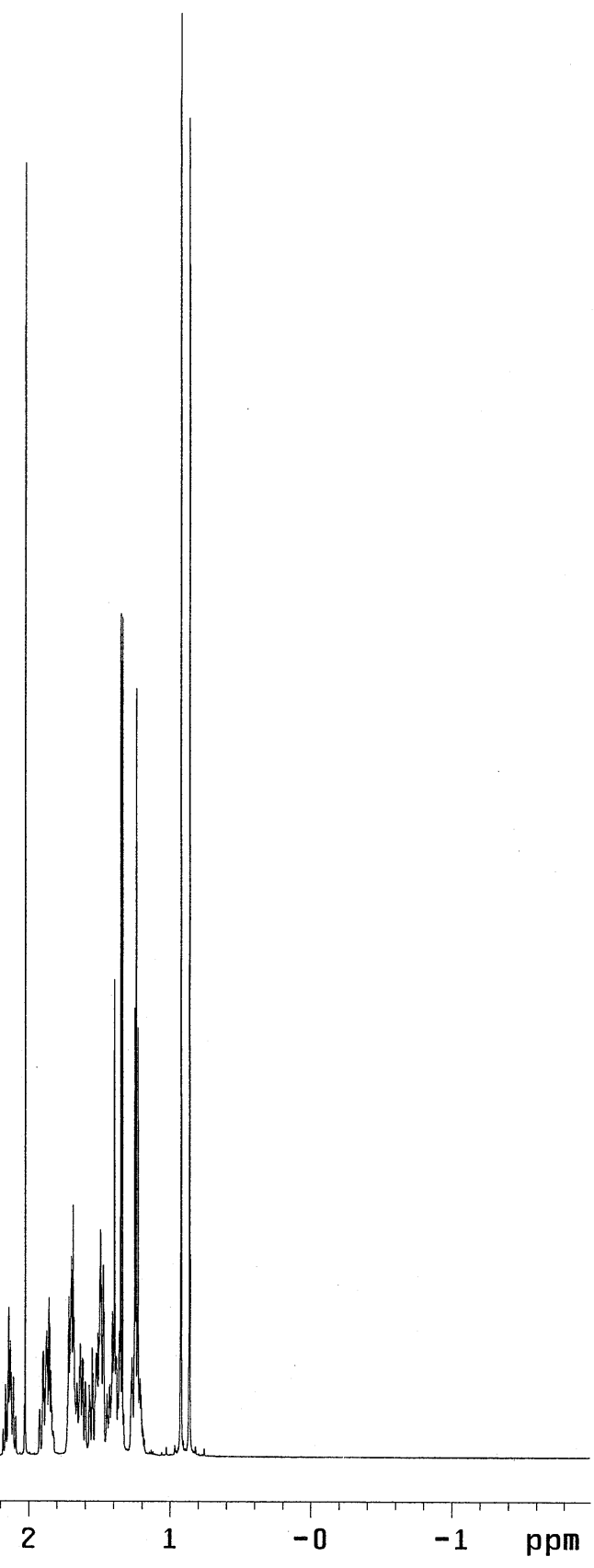




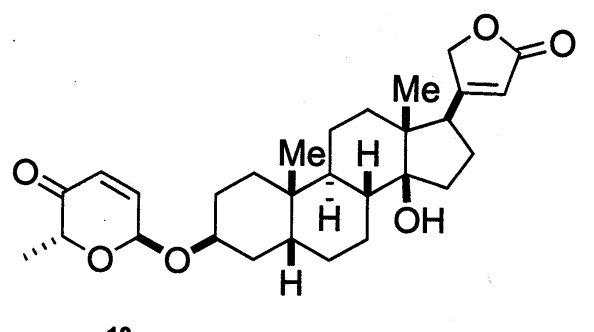

${ }^{13} \mathrm{C} \mathrm{NMR} \mathrm{(150} \mathrm{MHz,} \mathrm{CDCl}_{3}$ ) 

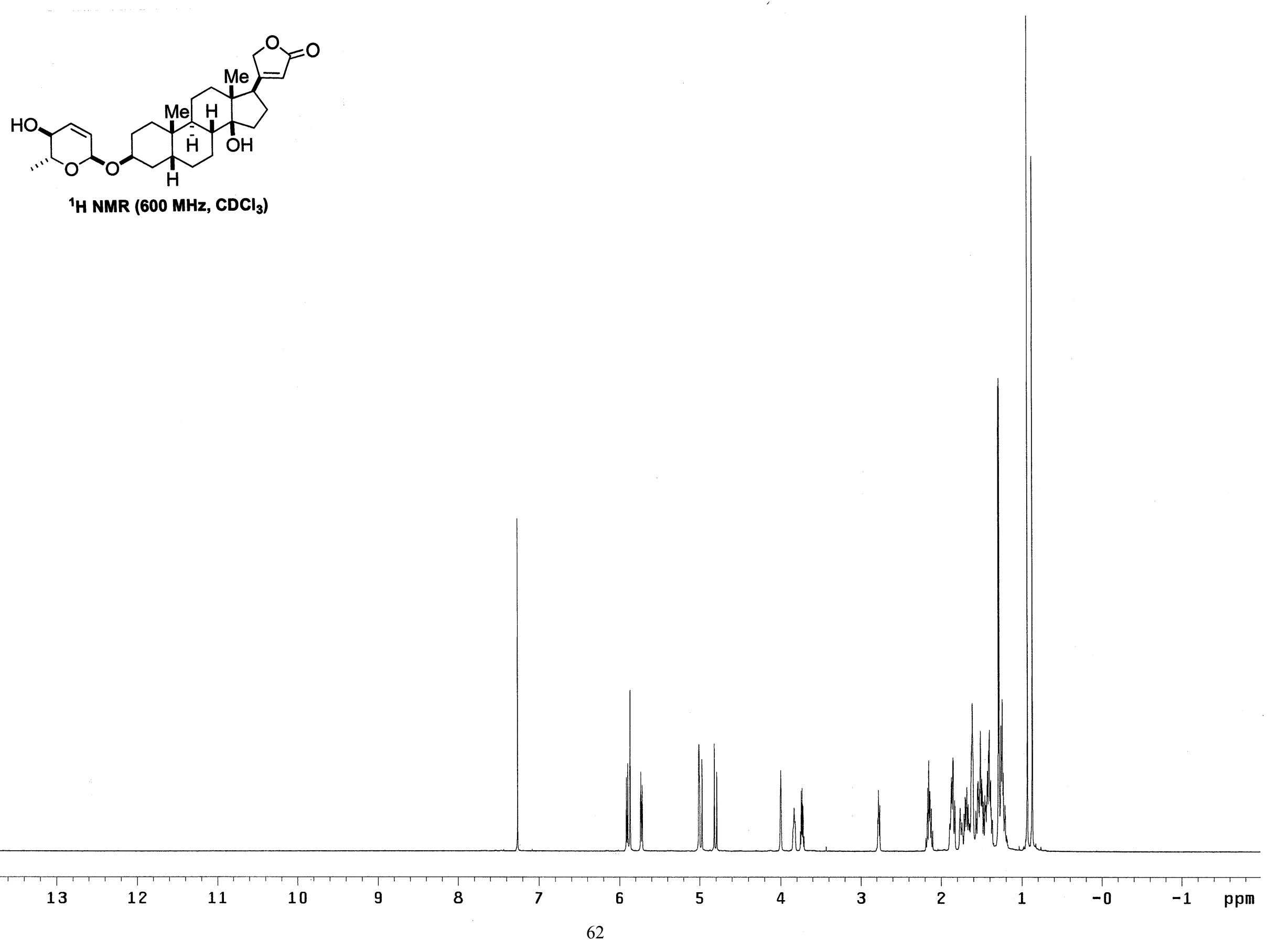


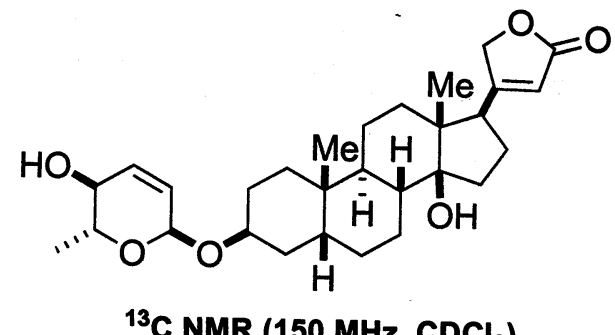

${ }^{13} \mathrm{C} \mathrm{NMR}\left(150 \mathrm{MHz}, \mathrm{CDCl}_{3}\right.$ )
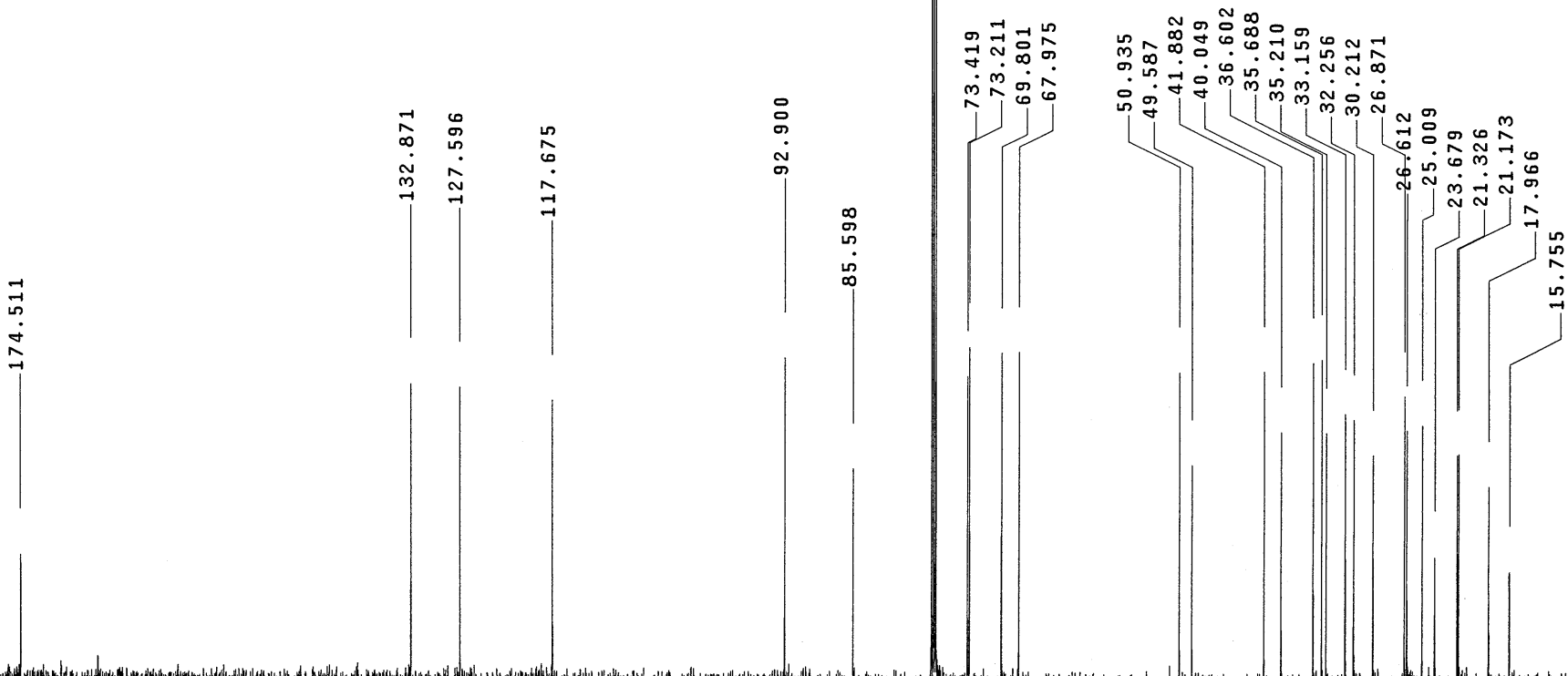

:

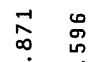

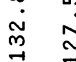

ले

.

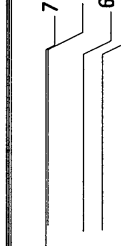<smiles></smiles>

กั
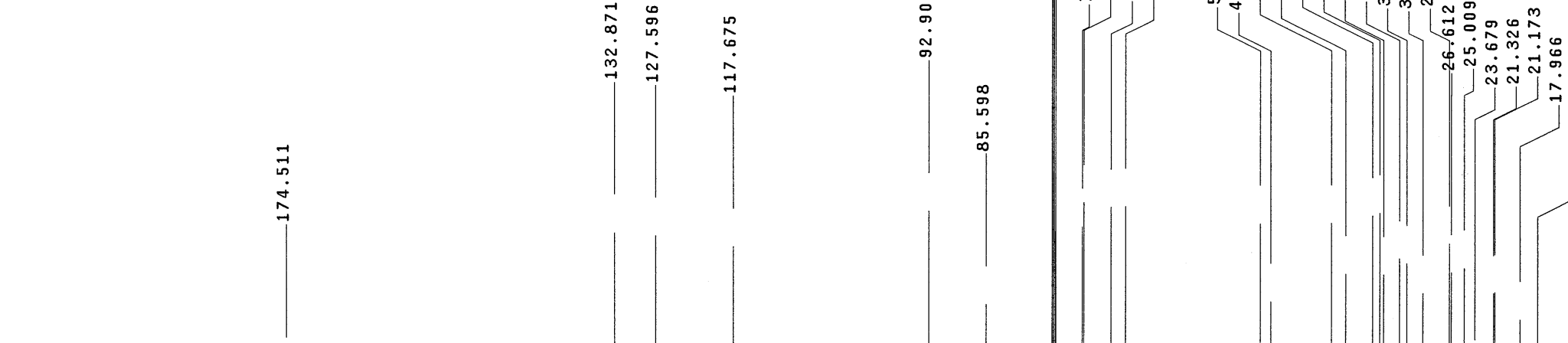


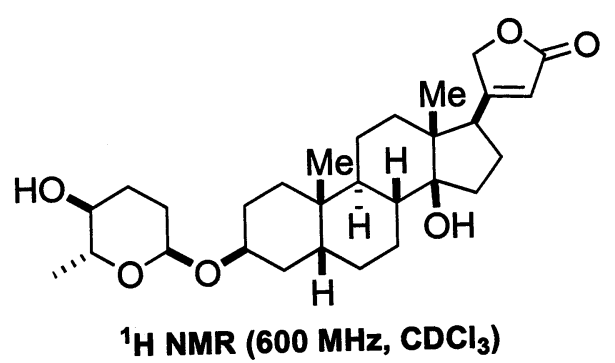

${ }^{1} \mathrm{H}$ NMR (600 MHz, $\mathrm{CDCl}_{3}$ )

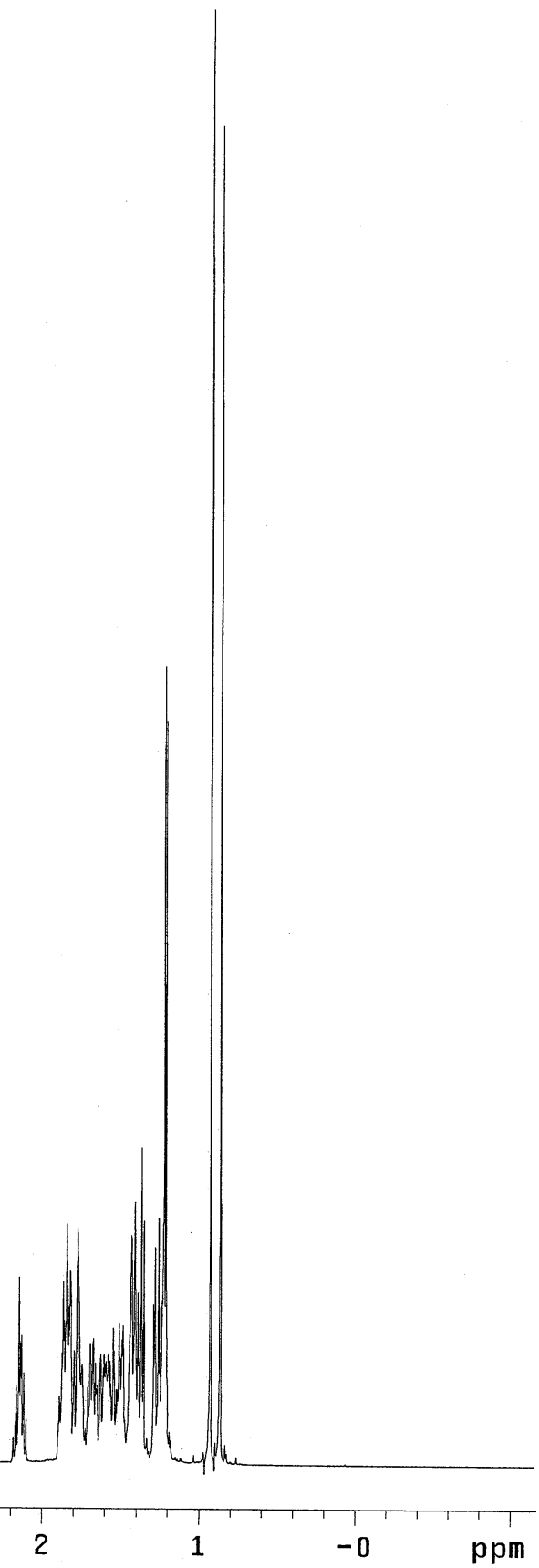




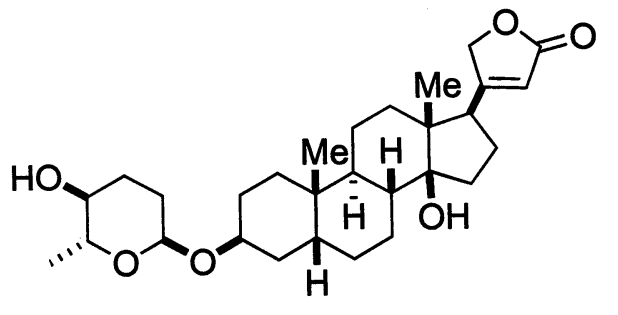

${ }^{13} \mathrm{C}$ NMR (150 MHz, $\left.\mathrm{CDCl}_{3}\right)$ 


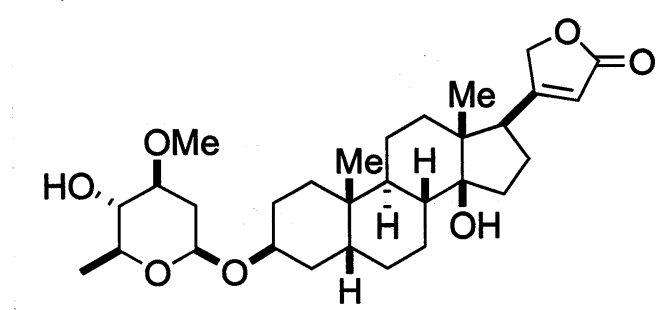

${ }^{1} \mathrm{H}$ NMR (600 MHz, $\mathrm{CDCl}_{3}$ )

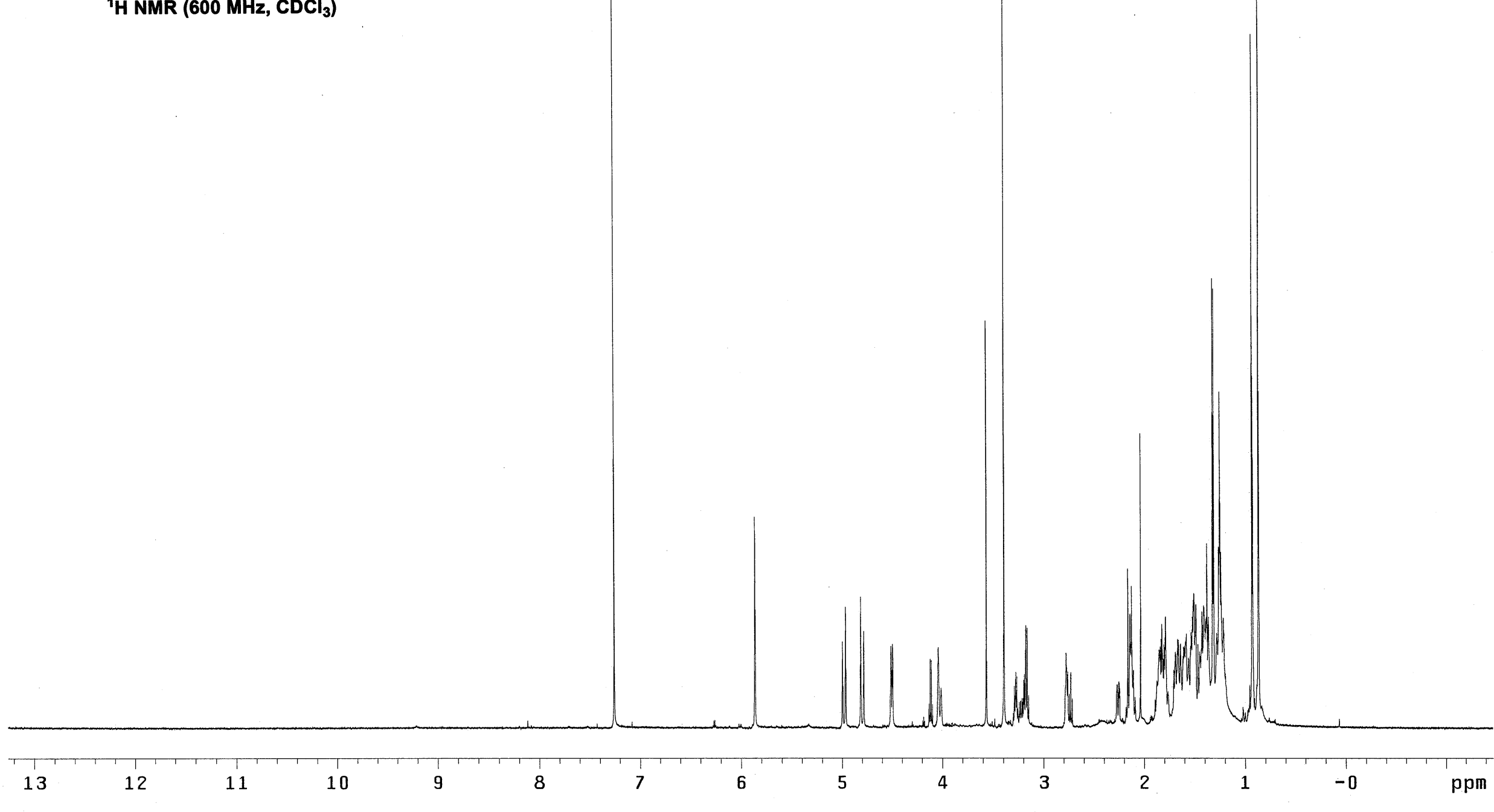




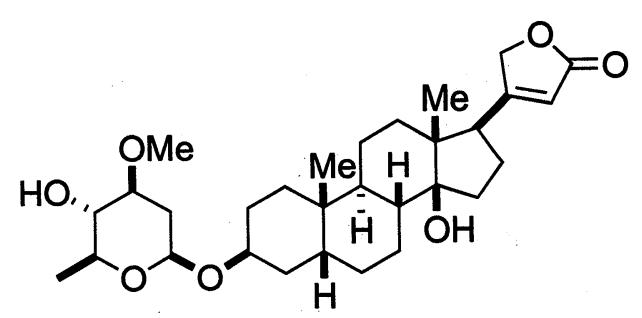

$\left.{ }^{13} \mathrm{C} \mathrm{NMR} \mathrm{(150} \mathrm{MHz,} \mathrm{CDCl}_{3}\right)$

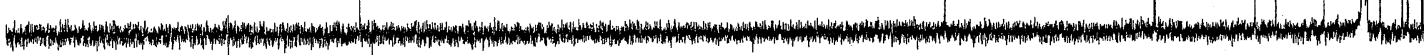

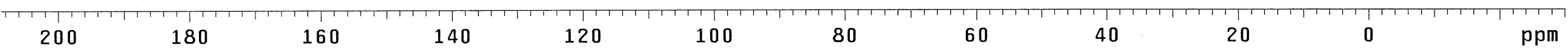

